\title{
The development of layered photonic band gap structures using a micro-transfer molding technique
}

\author{
By \\ Kevin Jerome Sutherland \\ A thesis submitted to the graduate faculty \\ in partial fulfillment of the requirements for the degree of \\ MASTER OF SCIENCE
}

Major: Materials Science and Engineering

Major Professor: Dr. K. P. Constant

Iowa State University

Ames, Iowa

2001 


\title{
Graduate College
}

Iowa State University

This is to certify that the Master's thesis of

\author{
Kevin Jerome Sutherland
}

has met the thesis requirements of Iowa State University

\author{
Major Professor
}

For the Major Program

For the Graduate College 


\section{TABLE OF CONTENTS}

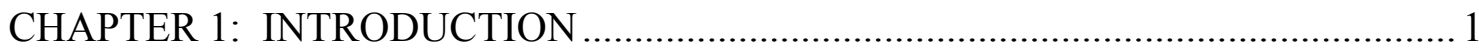

Scope and Importance of the Research........................................................... 1

Background Definitions ............................................................................... 3

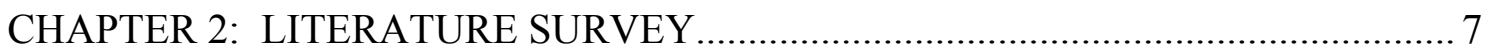

Photonic Band Gap Structure................................................................... 7

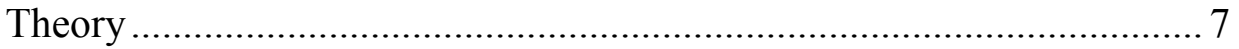

Layered Structures ........................................................................ 14

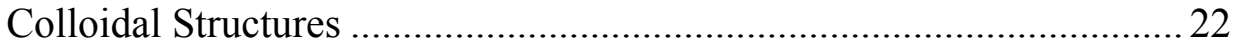

Application and Devices ......................................................................... 28

Optical Waveguides ........................................................................... 28

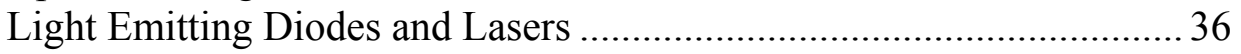

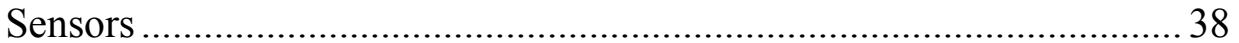

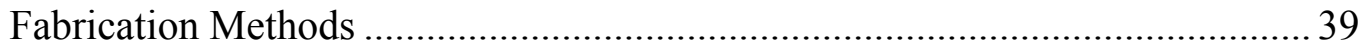

Laser Dye with Scatterers .............................................................................. 42

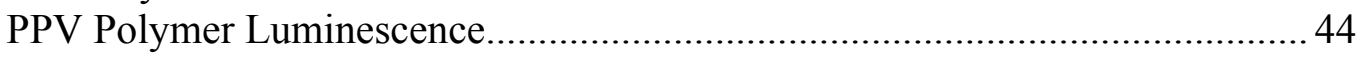

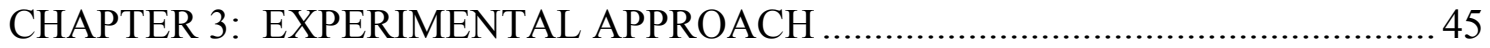

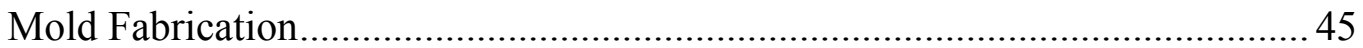

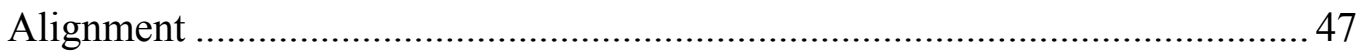

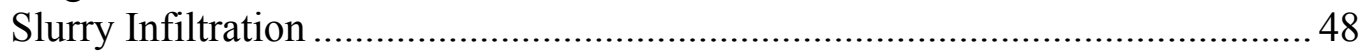

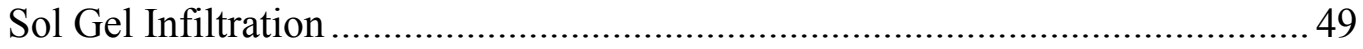

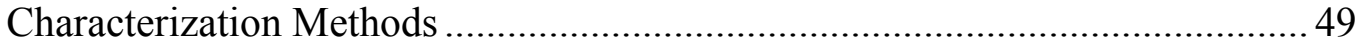

CHAPTER 4: RESULTS AND ACCOMPLISHMENTS …………………..............51

Experimental Equipment ……………………………................................ 51

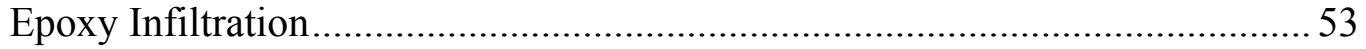

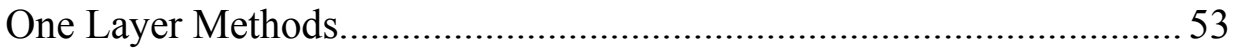

Multiple Layer Methods ................................................................... 53

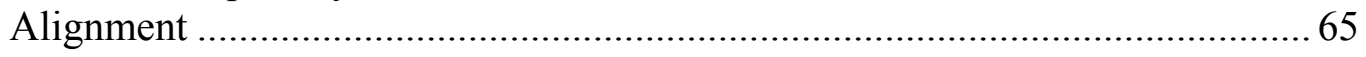

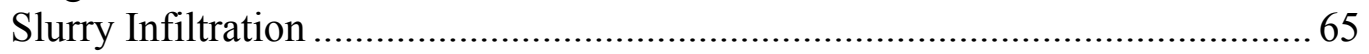

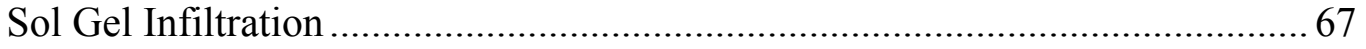

Other Analysis Methods ……………………………….............................. 71

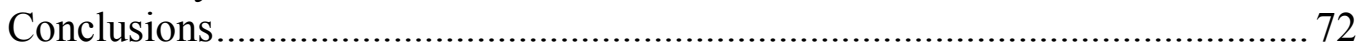

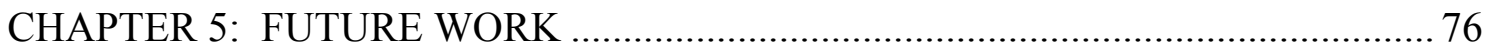

Micro Transfer Molding ………………………………........................... 76

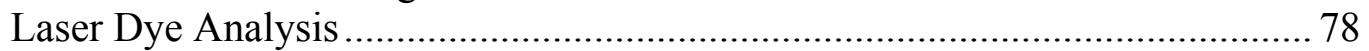

Summary of Future Work ............................................................................. 80

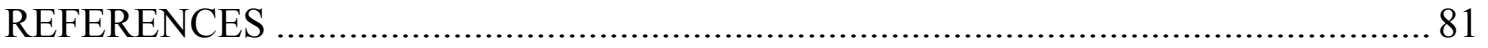

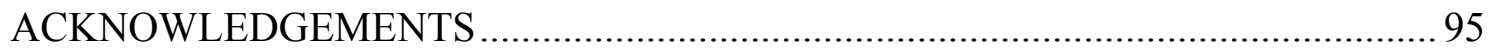




\section{CHAPTER 1: INTRODUCTION}

Photonic band gap (PBG) crystals are periodic dielectric structures that manipulate electromagnetic radiation in a manner similar to semiconductor devices manipulating electrons. Whereas a semiconductor material exhibits an electronic band gap in which electrons cannot exist, similarly, a photonic crystal containing a photonic band gap does not

allow the propagation of specific frequencies of electromagnetic radiation. This phenomenon results from the destructive Bragg diffraction interference that a wave propagating at a specific frequency will experience because of the periodic change in dielectric permitivity. This gives rise to a variety of optical applications for improving the efficiency and effectiveness of opto-electronic devices. These applications are reviewed later. Several methods are currently used to fabricate photonic crystals, which are also discussed in detail. This research involves a layer-by-layer micro-transfer molding ( $\mu \mathrm{TM})$ and stacking method to create three-dimensional FCC structures of epoxy or titania. The structures, once reduced significantly in size can be infiltrated with an organic gain media and stacked on a semiconductor to improve the efficiency of an electronically pumped light-emitting diode.

\section{Scope and Importance of the Research}

Photonic band gap structures have been proven to effectively create a band gap for certain frequencies of electro-magnetic radiation in the microwave and near-infrared ranges. The objective of this research project was originally two-fold: to fabricate a three dimensional (3-D) structure of a size scaled to prohibit electromagnetic propagation within the visible wavelength range, and then to characterize that structure using laser dye emission spectra. As a master mold has not yet been developed for the micro transfer molding technique in the visible range, the research was limited to scaling down the length scale as much as possible with the current available technology and characterizing these structures with other methods.

The fabrication of a visible wavelength PBG structure opens up the field to a wideranging set of new and exciting applications that have the potential to significantly impact a 
variety of technologies and industrial sectors from consumer products to military applications. One of the more exciting projected applications of photonic crystals is the production of optical circuits by manipulating light with optical waveguides. Currently, fiber-optic signals must be converted to electronic signals in order to be analyzed by computers or other devices connected to the optical line. This slows the signal considerably and the electronic circuitry is fairly inefficient as well. A device that runs completely on light would revolutionize technology in several areas, leading eventually to an all optical network (AON). The major problem with this application is the ability to manipulate light on a small enough scale. Optical waveguides currently operate on the principle of Total Internal Reflection (TIR), which occurs because an optical fiber possesses a higher refractive index than the surrounding media. Light will completely reflect off of the internal walls of the fiber if the photons are incident on the fiber wall at an angle less than a critical angle. Incident light greater than this angle will be allowed to transmit out of the fiber and be lost. This creates the problem of not being able to turn the light over small distances as that would cause the light to be incident on the fiber walls at too large of an angle. Photonic crystals are proposed as a possible solution to this problem. Adding a defect in a PBG structure opens a path along which electro-magnetic radiation can propagate. Because the PBG structure can be tailored to completely reflect certain frequencies, it is possible to turn corners with light in a distance on the order of the light's wavelength. Also, the light propagates in air, which lowers the absorption losses that occur in optical fibers.

Another exciting application of PBG structures is the use of these crystals to greatly improve the efficiency of lasers and light-emitting diodes. A photonic crystal infiltrated with gain media can improve the output of a light-emitting device in different ways. A onedimensional periodic dielectric-gain media structure will have a higher-power output frequency at the band gap edge because of the reflection of the radiation back and forth between each gain layer, increasing the stimulated emission four-fold. A two dimensional photonic crystal will also improve the emission efficiency of gain media by inhibiting spontaneous emission out of the lasing plane and causing the beam to be emitted along a defect path in the structure. The current research worked toward the ability to utilize a three- 
dimensional photonic crystal layer to direct the spontaneous light to produce a light-emitting diode with a considerable increase in efficiency and peak intensity.

A newer area of application interest deals with the use of these PBG materials as sensors. The size of the lattice parameter and the index of refraction difference of the PBG structure affect the transmission properties of the crystal. Thus temperature dependent index of refraction materials can be used to create a thermometer or temperature sensor and electrostrictive materials can be used to develop an electrical sensor. Other areas of interest concerning PBG materials include high-efficiency microwave antennas, wavelength filters and frequency disguise and recognition devices.

Completely developed PBG materials will introduce many changes in several areas of the electronics industry. Success in this research will aid in the development of a highefficiency light-emitting diode, utilizing a three-dimensional PBG structure to confine the emission of the gain media.

\section{Background Definitions}

In the literature survey that follows, an understanding of these definitions is useful. A minimal description is provided for each subject.

Reciprocal Lattice Vectors: Determined by Bragg diffraction in a crystal that define the reciprocal lattice space of that crystal. The primitive lattice vectors of the reciprocal lattice are given by $^{147}$ :

$$
b_{1}=2 \pi \frac{a_{2} \times a_{3}}{a_{1} \cdot a_{2} \times a_{3}} \quad b_{2}=2 \pi \frac{a_{3} \times a_{1}}{a_{1} \cdot a_{2} \times a_{3}} \quad b_{3}=2 \pi \frac{a_{1} \times a_{2}}{a_{1} \cdot a_{2} \times a_{3}}
$$

where $a_{1}, a_{2}, a_{3}$ are the primitive vectors of the regular crystal lattice and $b_{1}, b_{2}, b_{3}$ are the primitive vectors of the reciprocal lattice. These primitive vectors are used to determine the reciprocal lattice vector $\mathrm{G}$ as defined below: 


$$
G=v_{1} b_{1}+v_{2} b_{2}+v_{3} b_{3}
$$

where $v_{1}, v_{2}, v_{3}$ are integers.

Reciprocal Lattice: The reciprocal lattice is used to map the diffraction pattern of a regular crystal. This is useful in determining optical properties of different atomic arrangements.

Brillouin Zones (BZ): The Brillouin zone is defined as the enclosure of planes that perpendicularly bisect the midpoint of the primitive reciprocal lattice vectors. It is useful for defining the diffraction condition of different wavevectors. Any wavevector drawn from the origin of the unit cell of the reciprocal lattice to the Brillouin zone edge will be diffracted by the crystal, which leads to the formation of a band structure. Several of these directions are labeled with letters $(\Gamma, W, X, L, U)$, which makes it easier to visualize a band structure when analyzing several different frequencies at different directions in the crystal. These directions, along with a typical photonic band structure are shown in figure 2.3.

Filling Fraction: The fraction of material that comprises a certain structure. If a sample is made to be $70 \%$ titania and $30 \%$ air, it is said to have a titania filling fraction of 0.7 .

Wavenumbers, Frequency, and Wavelength: Several terms and types of units are used to classify electromagnetic waves and the size of the PBG structure. Frequency, (Hz or oscillations/second) is related to wavelength, $(\mu \mathrm{m})$ and wavenumber $\left(\mathrm{cm}^{-1}\right)$ by the following equations:

$$
\lambda=\frac{c}{v}=\frac{2 \pi}{k}
$$


where $\lambda$ represents wavelength, $\mathrm{c}$ is the speed of light, $v$ is frequency and $\mathrm{k}$ represents wavenumber. Common values in a range pertinent to this research for each of these variables are shown in the following table 1.1.

Table 1.1 - Relationship between wavelength, frequency and wavenumber

\begin{tabular}{|c|c|c|c|}
\hline Region in Spectrum & $\begin{array}{c}\text { Wavelength } \\
\text { (nanometers) }\end{array}$ & $\begin{array}{c}\text { Frequency } \\
(\text { terahertz-10 }\end{array}$ & $\begin{array}{c}\text { Wavenumber } \\
\left(\mathrm{cm}^{-1}\right)\end{array}$ \\
\hline Blue-Violet & 300 & 1000 & 62800 \\
\hline Red & 600 & 500 & 31400 \\
\hline IR & 3000 & 100 & 6280 \\
\hline IR (Curent Sample Size) & 6100 & 49 & 838 \\
\hline
\end{tabular}

Photonic band gap: The band of frequencies of electro-magnetic radiation that are not allowed to pass through a PBG material. The mid-gap frequency and size of the gap are determined by the periodicity, dielectric filling fraction, and index of refraction of the material in question. Table 1.2 is shown below that provides a few different calculated band gap values for these different variables.

Table 1.2 - Band gap sizes and frequencies for different parameters of layered structures

\begin{tabular}{|c|c|c|c|c|}
\hline $\begin{array}{c}\text { Index of Refraction } \\
\text { of Titania }\end{array}$ & $\begin{array}{c}\text { Filling } \\
\text { Fraction }\end{array}$ & $\begin{array}{c}\text { Periodicity of bars } \\
(\mu \mathrm{m})\end{array}$ & $\begin{array}{c}\text { PBG Mid-gap } \\
\text { Wavelength }(\mathrm{nm})\end{array}$ & PBG Width (nm) \\
\hline 2.2 & 0.4 & 0.25 & 560 & 110 \\
\hline 2.5 & 0.4 & 0.25 & 610 & 140 \\
\hline 2.2 & 0.35 & 0.29 & 620 & 120 \\
\hline 2.5 & 0.35 & 0.29 & 670 & 160 \\
\hline 2.2 & 0.33 & 0.3 & 630 & 120 \\
\hline 2.2 & 0.25 & 0.4 & 750 & 130 \\
\hline 2.5 & 0.25 & 0.4 & 850 & 200 \\
\hline 2.2 & 0.33 & 0.45 & 950 & 190 \\
\hline 2.2 & 0.33 & 0.75 & 1570 & 290 \\
\hline 2.2 & 0.4 & 2.5 & 5600 & 1100 \\
\hline 2.5 & 0.4 & 2.5 & 6100 & 1400 \\
\hline
\end{tabular}


Kossel Lines: Dark rings and arcs resulting from the illumination of a single colloidal crystal with a monochromatic laser beam. They are formed from the light that satisfies the Bragg condition along specific angular cones and can be used to analyze single crystal colloid band gaps.

s-Polarization: Incident electromagnetic waves on a surface are said to be spolarized if the electric field component of the wave is parallel to the surface.

p-Polarization: Incident electromagnetic waves on a surface are said to be ppolarized if the electric field component of the wave is perpendicular to the surface.

Electroluminescence (EL): Electroluminescent materials emit photons when excited by electron injection. This phenomenon is demonstrated in semiconductor lasers where electric current is used to generate a coherent beam of photons.

Photoluminescence (PL): Photoluminescent materials emit photons when excited (pumped) by a higher energy light source. Electrons in the photoluminescent material are excited into higher energy states by the incident light and will emit photons as they return to the original lower energy states. 


\title{
CHAPTER 2: LITERATURE SURVEY
}

\author{
Photonic Band Gap Structure
}

\section{Theory}

The first suggestion that one could produce a three-dimensional forbidden gap in the electromagnetic spectrum was made in 1987 by Eli Yablonovitch ${ }^{1}$. One-dimensional structures had already been created by periodically modulating the index of refraction in a layered structure. This structure had been used in distributed-feedback lasers and FabryPerot resonators since it is nearly an ideal reflective mirror for light propagating perpendicular to the alternating layers. Yablonovitch therefore suggested that for a wavelength $\lambda$, a full three-dimensional structure with a spatial periodicity of $\lambda / 2$ would open a forbidden gap in the electromagnetic spectrum at that wavelength in all directions. His calculations also suggested that the first Brillion zone (BZ) of the photonic band gap (PBG) structure in reciprocal space should be as close to spherical as possible, and face centered cubic structures were proposed as the most common structure to have a nearly spherical first BZ. Yablonovitch predicted that this concept could be utilized to develop more efficient lasers, bipolar junction transistors (BJT's), and solar cells because of the ability to manipulate spontaneous emission.

Later that year, S. John provided a theoretical explanation for the existence of a PBG in 3-D periodic structures ${ }^{2}$. One suggestion was that Anderson localization has an effect on electromagnetic radiation in a disordered dielectric contrast medium similar to the effect electrons see in a disordered varying potential. John also discussed Bragg reflection and proposed this to be the mechanism by which electro-magnetic (EM) waves decay exponentially with distance into the medium. He then showed calculations that determined the necessary dielectric contrast needed to create a gap in a FCC periodic lattice. This is shown in figure 2.1 . 

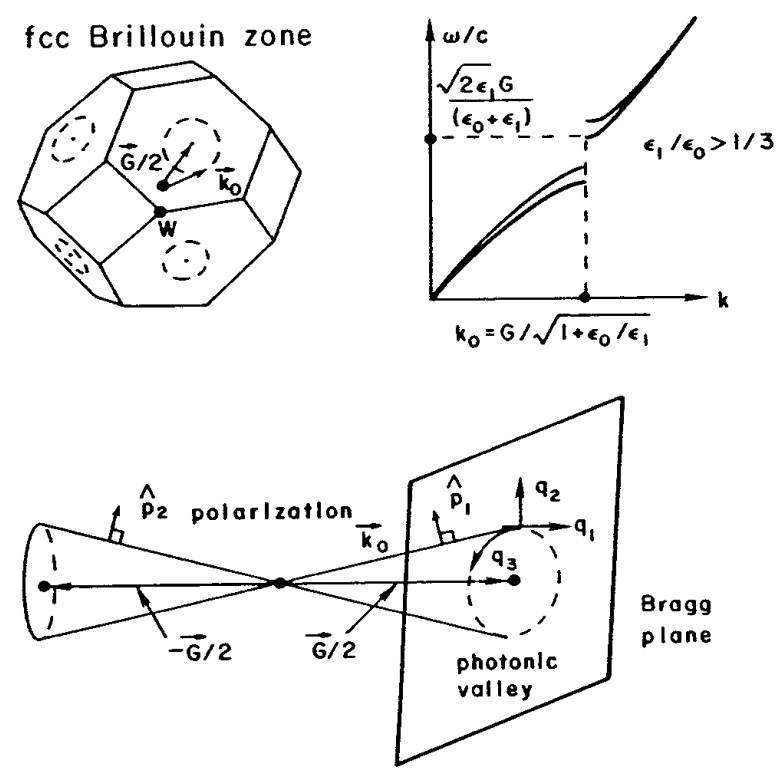

Figure 2.1 - A gap in optical modes caused by Bragg reflection effects. Calculations show that the dielectric coefficient of one material must be at least three times that of the other in order to produce the gap ${ }^{2}$

In 1989, E. Yablonovitch and T. J. Gmitter constructed a band gap structure in the microwave frequencies to test the theoretical results ${ }^{3}$. They varied dielectric contrast and volume filling fraction of their periodic FCC dielectric structures. Two types of construction were used: one in which the "atoms" were polycrystalline alumina balls contained in a low index matrix, and one in which air "atoms" were drilled from a high index matrix. They found that most of their attempts resulted in semi-metal band behavior in which portions of the valence band would overlap other parts of the conduction band. Success was obtained, however for a structure of air atoms in a low-loss dielectric matrix with a microwave refractive index of $\sim 3.5$, and they found the optimal volume fraction of the air "atoms" to be $86 \%$. The material used was commercially available Emerson \& Cumming Stycast-12. The experimental structure and its band diagram are shown in figures 2.2 and 2.3. These structures operating at microwave frequencies could be used to predict the behavior of similar samples on the scale of light frequencies. The model did not account for every possible polarization and direction of incidence, but it still provided an excellent insight of the necessary parameters needed to create a PBG. 


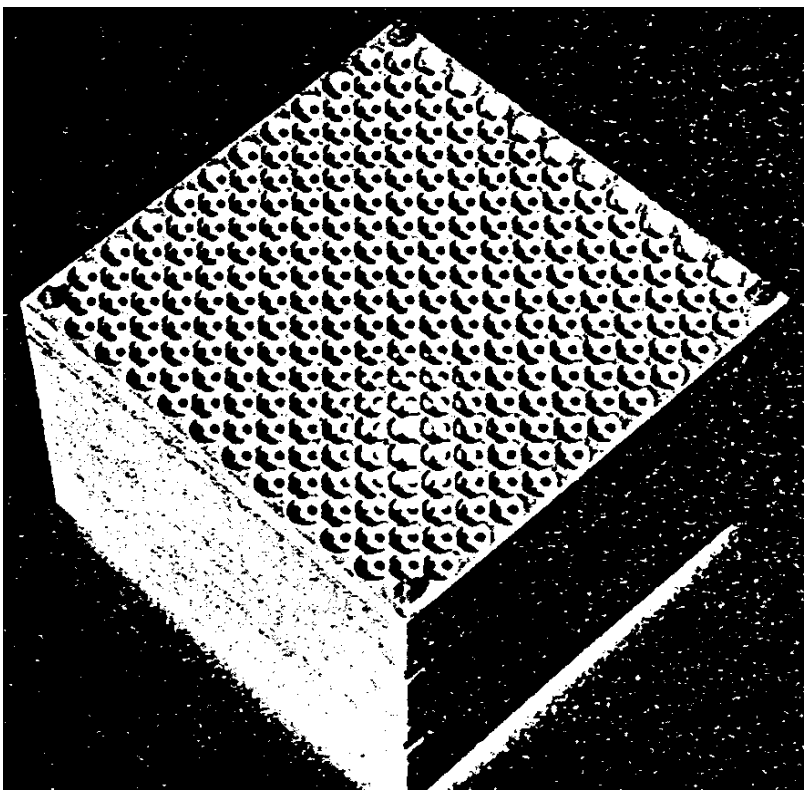

Figure 2.2 - The original PBG structure tested in the microwave frequencies ${ }^{3}$

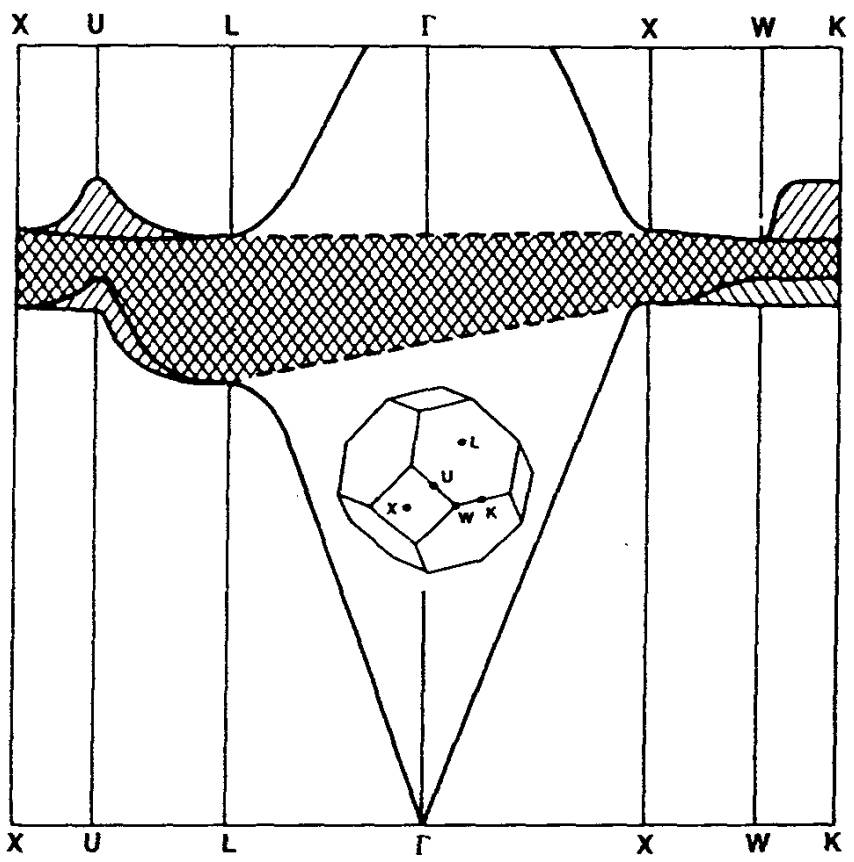

Figure 2.3 - Experimental band diagram for a $86 \%$ spherical-air-atom crystal. The lines sloping downward to the right represent s-polarized EM radiation, and the lines sloping downward to the left represent p-polarized modes ${ }^{3}$ 
The above experiments were verified theoretically by K.-M. Ho et al. in $1990^{4}$. His group solved Maxwell's equations exactly, taking into account the vector nature of the electromagnetic field. The results of the calculations suggested that a FCC structure possessed only a pseudo-gap rather than a full PBG, as a few propagating modes overlapped in the FCC band structure. The calculations proved however, that a diamond structure would indeed result in a full PBG. The calculations were performed for both $\mathrm{s}$ and p-polarizations on air spheres and dielectric spheres. The resulting equations optimized the structure, refractive index, and filling ratio parameters for the fullest, largest band gap. The best calculated structure found was the diamond structure of dielectric spheres with a refractive index of 3.6 and a filling ratio of $34 \%$. The theoretical band diagrams for the FCC and diamond cases are shown in figures 2.4 and 2.5 . The calculations actually showed that a fairly wide range of filling ratios and refractive indexes for a diamond lattice would open a full PBG. Index of refraction contrast could be as low as 2 while the filling ratio of dielectric spheres ranged from around 0.2 to 0.67 and air spheres from 0.37 to 0.95 to still obtain a photonic band gap. The calculations made for the FCC lattice contradicted the experimental work of Yablonovitch and Gmitter. Ho et al. attributed this to the inadequate resolution of the experimental setup. The equipment was claimed to be unable to observe the degeneracy of the bottom of the s gap and the top of the $\mathrm{p}$ gap at the $\mathrm{W}$ point in the BZ.

E. Yablonovitch et al. recognized that their previous FCC experiment may not have accounted for the degeneracy at the $\mathrm{W}$ point in the BZ, but they developed a lattice utilizing a different shaped atom ${ }^{6}$. They used a Wigner-Seitz (WS) unit cell of the FCC lattice, which was created by drilling holes at a 35.26 degree angle rotated 120 degrees from each other around a central axis as shown in figure 2.6. Stycast-12 was used again as the surrounding matrix for the air "atoms" with a microwave index of refraction of 3.6. The same experimental setup was used to test the new structure and the experiment proved that the WS cell structure could still develop a full three-dimensional PBG, depending on the shape of the atoms created. This was an important result because the it could be fabricated much more easily than the diamond structure, which is slightly more complicated. 


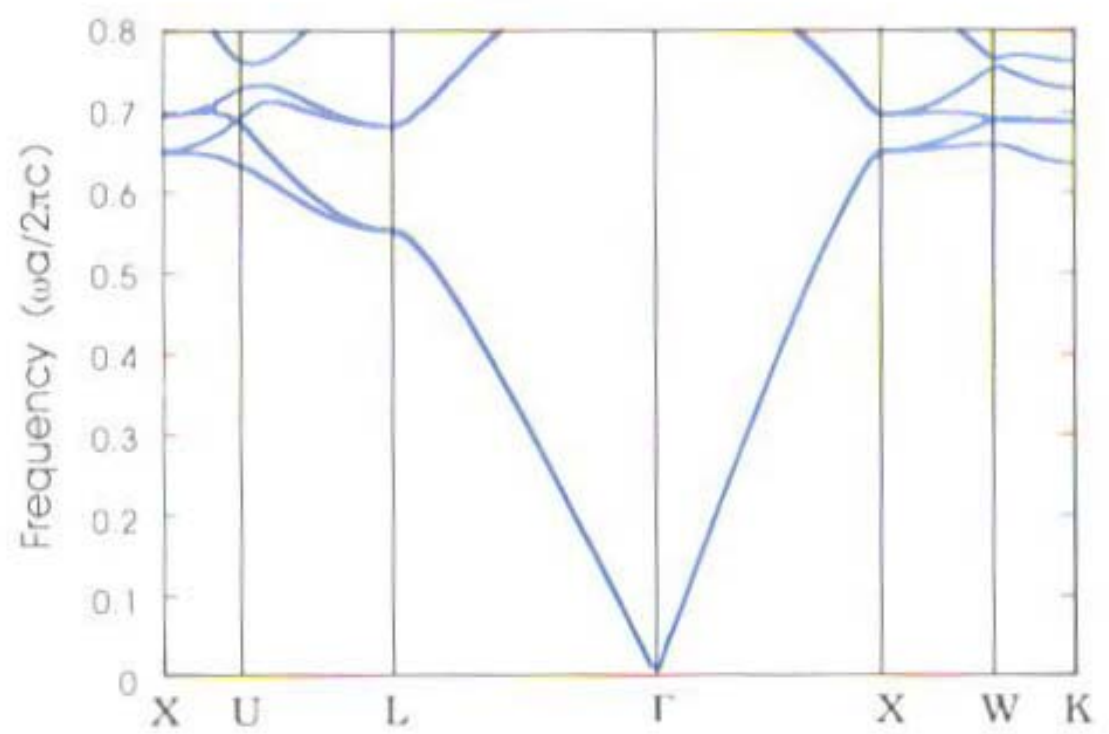

Figure $2.4-$ Calculated band structure for a FCC Structure ${ }^{148}$

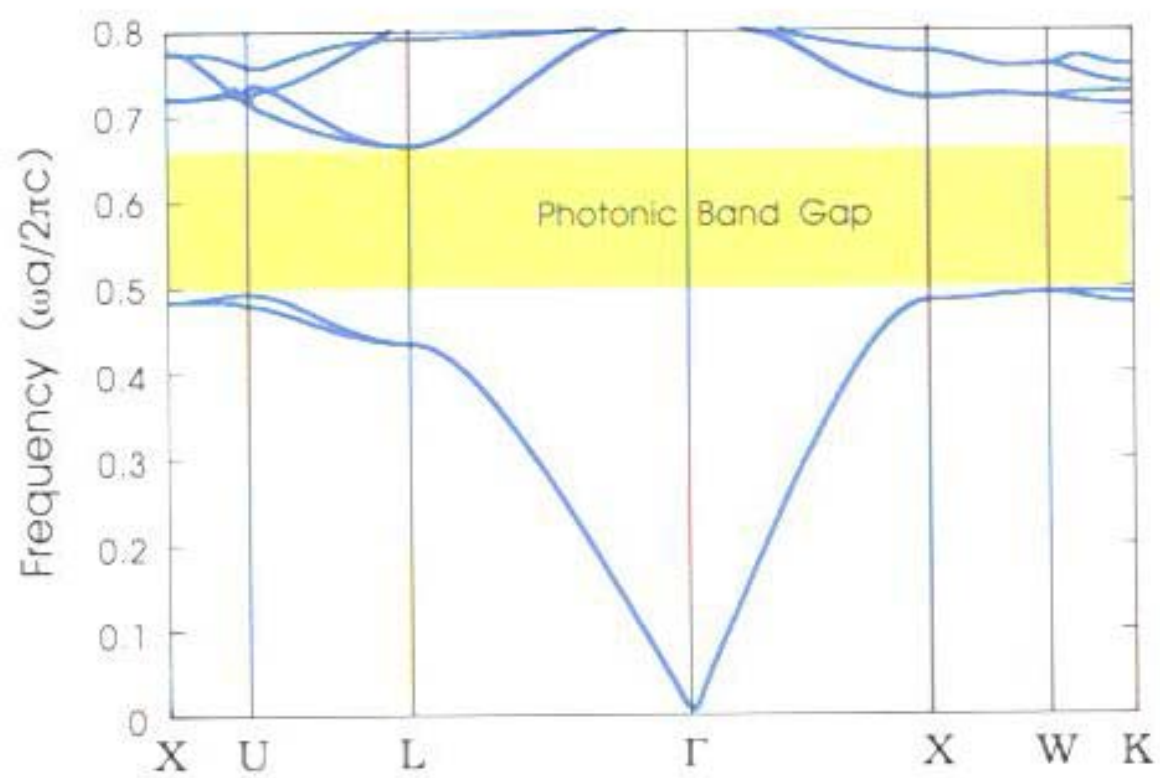

Figure 2.5 - Calculated band structure for a diamond dielectric matrix consisting of spheres of refractive index of 3.6 in air background and a filling ratio of 34\%. Frequency is given in units of $\mathrm{c} / \mathrm{a}^{148}$ 

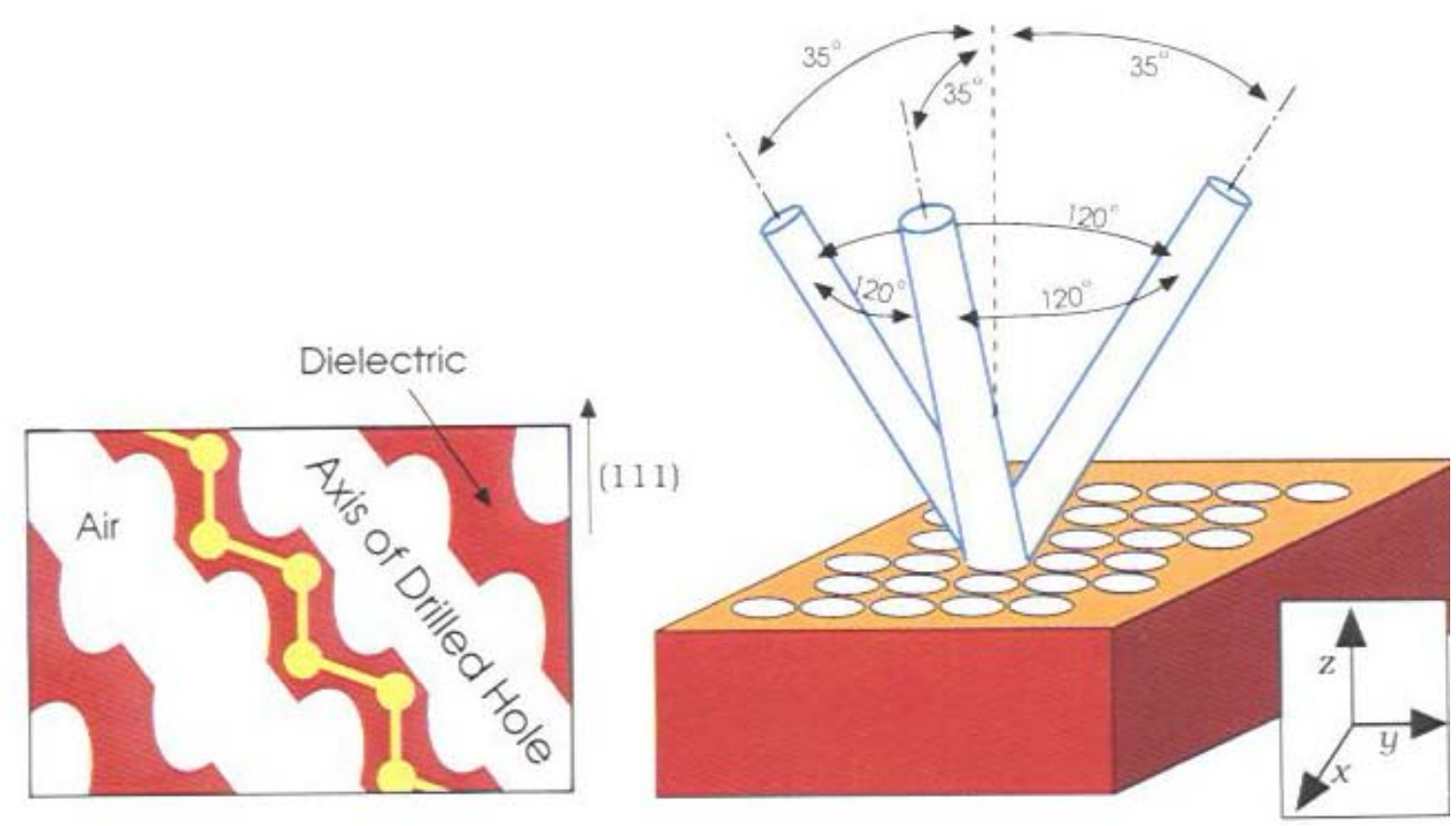

Figure 2.6 - Schematic diagram of the newly proposed structure, developing a full 3-D $\mathrm{PBG}^{148}$

In 1993, Yablonovitch discussed the possibility of doping a photonic crystal in the same manner in which a semiconductor is doped ${ }^{7}$. He proposed introducing a defect into the PBG structure that acts as an acceptor or donor for specific frequencies within the band gap. Adding dielectric material to an air site was expected to create a donor state and removing a section of the dielectric matrix was expected to create an acceptor state in the structure. The donor and acceptor states were tested and the resulting spectra are shown in figures 2.7 and 2.8. The size of the added defect affected the placement of the defect state within the band gap. This dependence is shown in figure 2.9. Acceptor states move across the gap from the valence band as the hole defect size increases and donor states move from the conduction band as dielectric defect size increases. Just as the ability to dope a semi-conductor is the essential ingredient to making functional devices, the ability to create defect states produces many potential applications for photonic crystals. 


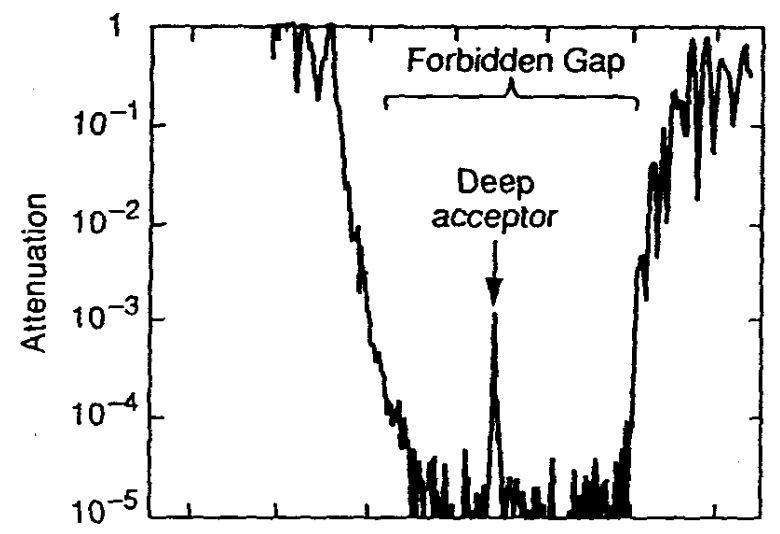

Figure 2.7 - Deep acceptor dopant ${ }^{7}$

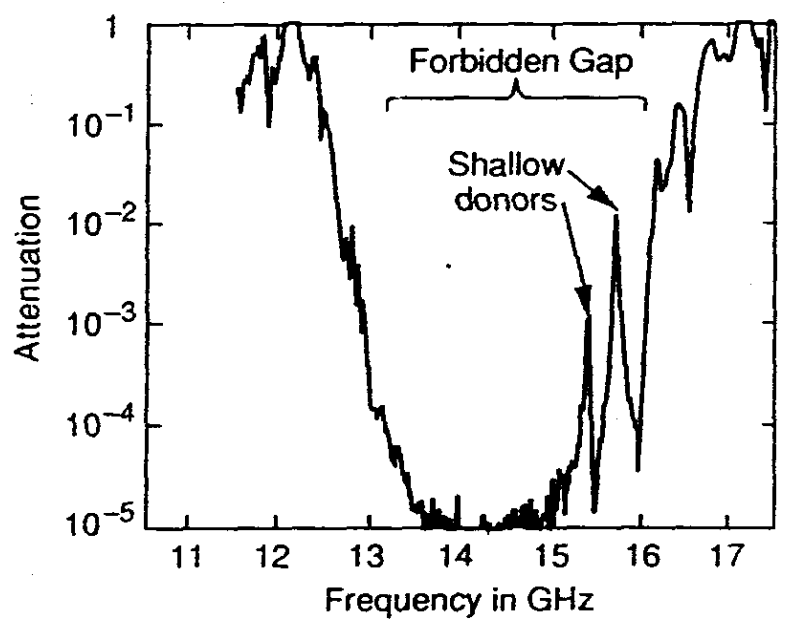

Figure 2.8 - Shallow Donor Dopants ${ }^{7}$

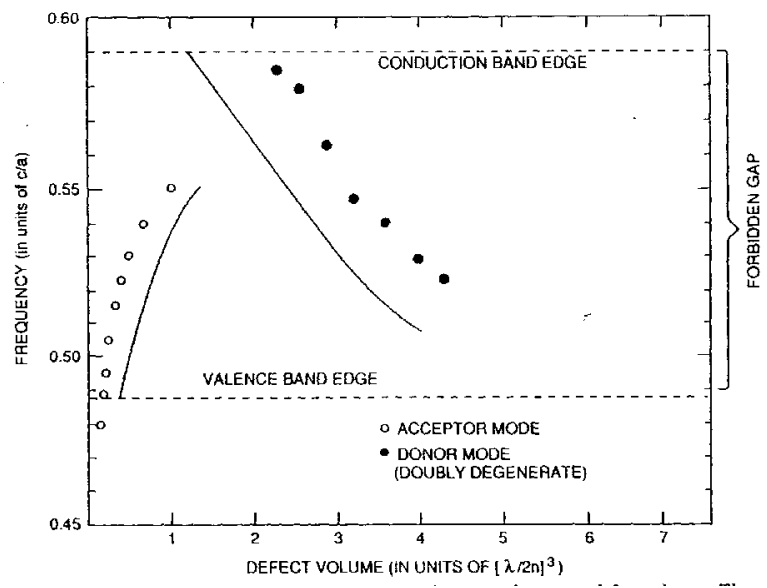

Figure 2.9 - Size dependence of defects on the placement of a defect state within a $\mathrm{PBG}^{7}$ 
M. Doosje et al. used the plane-wave expansion method in 2000 to further optimize different parameters in order to develop the ideal structure exhibiting a full, omni-directional PBG. $^{21}$ Similar to previous results, the final calculated structure was an inverse FCC matrix of air "atoms." The biggest difference in the new calculated structure was that the air atoms were connected to each other by air cylinders, which further reduced the volume fraction of dielectric material. They calculated the ideal physical parameters as follows: R/a must be greater than 0.177 , where $\mathrm{R}$ is the radius of each air sphere and a is the distance between them and the filling fraction $\phi=16.9 \pi\left(R_{C} / a\right)^{2}-26.7 \pi(R / a)^{3}$ where $R_{C}$ is the radius of the cylinder connecting adjacent air atoms. A picture of the structure is shown in figure 2.10.

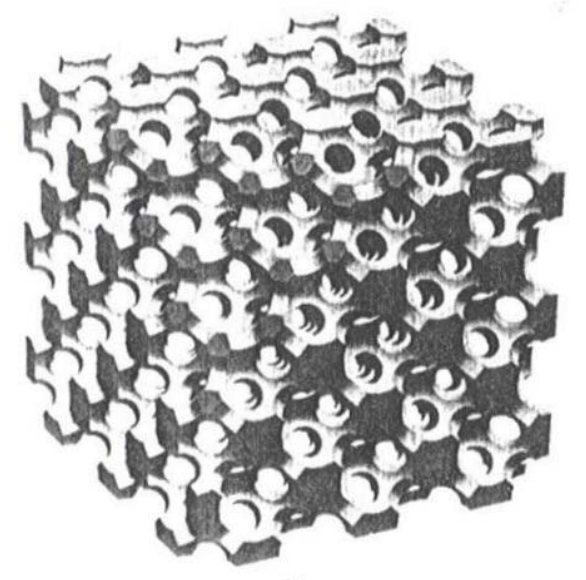

Figure 2.10 - Depiction of the ideal PBG structure calculated by M. Doojse et al. ${ }^{21}$

\section{Layered structures}

The Iowa State group, lead by K.-M. Ho, was the first to report the method of building a PBG structure from layers of dielectric rods ${ }^{22}$. Several calculations were made for different structure parameters: rod cross-sectional shape, index of refraction, c/a ratio, and bar overlap. The optimal structure they considered was made of alternating layers of dielectric rectangular rods. The layers were perpendicular to each other and every other layer was offset by half a period, creating a face-centered tetragonal lattice symmetry with a basis of two rods. The rods were spaced to create a filling ratio of $26.6 \%$ dielectric material with 
an index of refraction of 3.6. The proposed structure and band diagram are shown in figures 2.11 and 2.12. This PBG structure was an important discovery as it could be scaled to sizes operable in the visible wavelengths. At this point in time, the highest frequency experimental structures produced had been for less than $20 \mathrm{GHz}$.

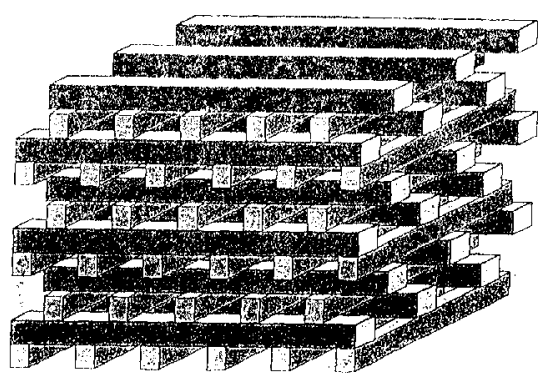

Figure 2.11 - Representation of the theoretical structure ${ }^{22}$

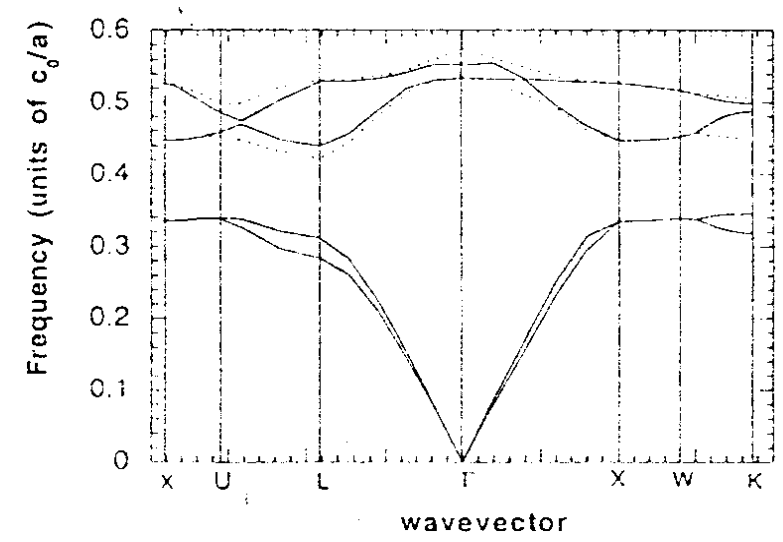

Figure 2.12 - The above structure's band diagram calculated for a filling fraction of $26.6 \%$ dielectric material in air with a refractive index of $3.6^{22}$

E. Ozbay et al. ${ }^{23}$ produced this layered structure experimentally, operating in the 100 $\mathrm{GHz}$ range soon after in 1994. An anisotropic etching of silicon by aqueous potassium hydroxide was used to pattern a structure with a bar thickness of $340 \mu \mathrm{m}$, a height of $390 \mu \mathrm{m}$, and a spacing thickness of $935 \mu \mathrm{m}$. The experimental crystal consisted of 28 -stacked wafers (7 unit cells), and the results of the transmission studies are shown in figure 2.13. The experimental data was in good agreement with the theoretical calculations. The group also measured attenuation as a function of number of layers in the structure. They found the 
relationship to be close to the theoretical expectation of $17 \mathrm{~dB}$ per unit cell (4 layers) and stated that 3 unit cells would be adequate for most applications. Low experimental attenuation past 3 unit cells was attributed to EM wave leakage around the crystal in the testing setup. He suggested that this production method could be used to make structures small enough to produce band gaps in the $3 \mathrm{THz}$ region.

E. Ozbay et al. ${ }^{24}$ also produced the layered structure experimentally also by stacking alumina rods (refractive index $\sim 3.1$ ) into the arrangement described previously. The experimental transmission data for this structure also correlated well to the theoretical calculations, displaying a band gap between 11.7 and $13.5 \mathrm{GHz}$. The experimental structure and its transmission data are shown in figures 2.14 and 2.15 .
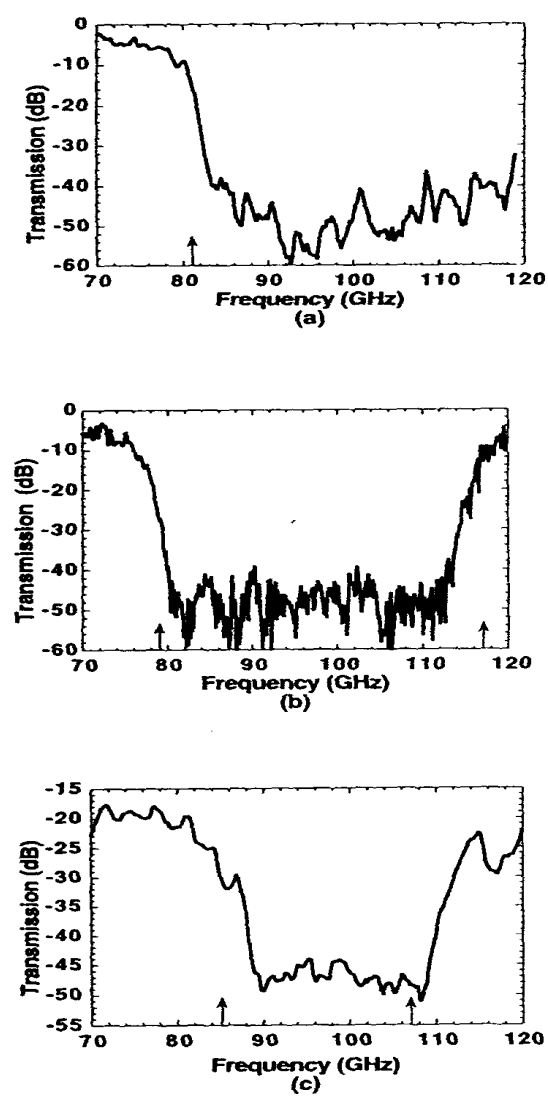

Figure 2.13 - Experimental band gap existing for EM waves: a) normal to wafer surfaces, b) 35 degrees with respect to the wafer normal, and c) parallel to wafer surfaces ${ }^{23}$ 


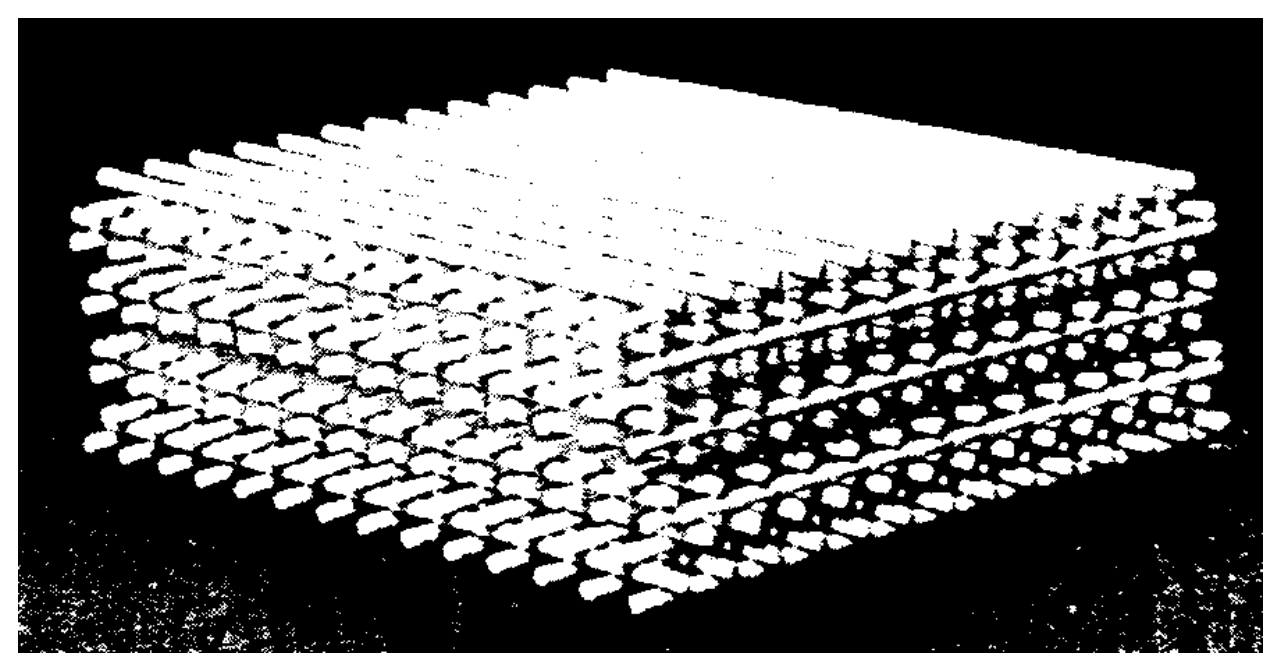

Figure 2.14 - Alumina rod structure. Unit consists of 16 layers of rods (13 or 14 rods in each layer $)^{24}$
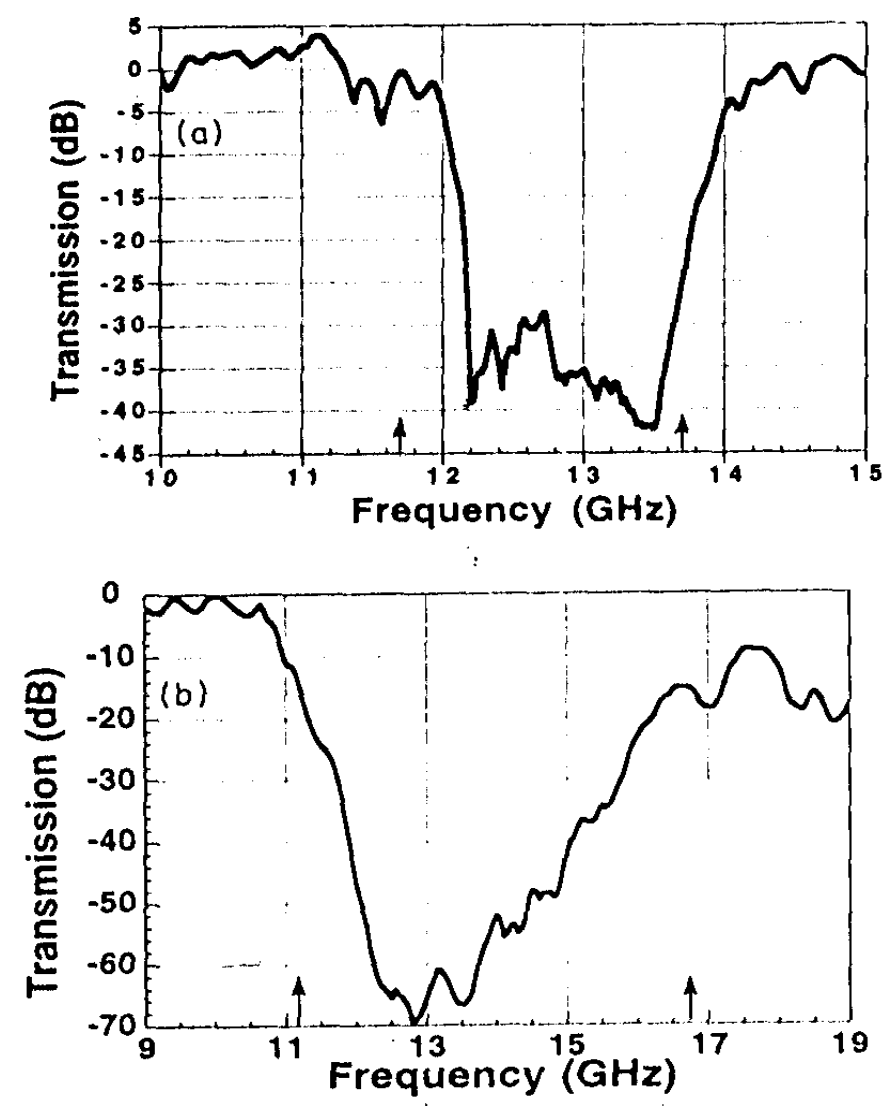

Figure 2.15 - Transmission plot is for above picture with E-field perpendicular to the rods ${ }^{24}$ 
A laser-write method for creating the layered bar PBG structures was by M. C. Wanke et al. ${ }^{28}$ With this process, it was possible to create a PBG crystal at frequencies of 2 THz. They used an additive laser chemical vapor deposition (LCVD) procedure, which is extremely flexible and allows the user to be able to introduce defects or change the structure in order to introduce certain properties in the crystal. The process was used to make the layered PBG structure by first depositing vertical layers, and then growing the horizontal layers, which formed a robust mechanical connection. The transmission results of the experimental structure exhibited a shallow band gap at around $2 \mathrm{THz}$. With the LCVD process, it was also possible to make more complex structures such as the diamond lattice. The procedure could be done around parts or free standing so that a substrate wasn't necessary. This was a very versatile method that could lead to several practical applications, but did not have a high enough resolution to be applied to visible wavelengths.

S. Noda et al., a group from Kyoto University in Japan ${ }^{25}$, was the next to use the layered bar stacking method to create a PBG material. In 1996, they constructed the layered structure with semiconductor processing technology. The process is depicted in figure 2.16. First an AlGaAs etch-stop layer $(1.0 \mu \mathrm{m})$ and GaAs layer $(1.5 \mu \mathrm{m})$ were grown on a GaAs substrate by molecular beam epitaxy (MBE) as shown in part (a) of figure 25. Stripe patterns (12.5 $\mu \mathrm{m}$ width and $15.5 \mu \mathrm{m}$ period) were then formed out of the GaAs by reactive ion etching (RIE) shown in (b). Two layers produced in this manner were then aligned 90 degrees from each other and stacked (c). One side of the AlGaAs stop-layer and GaAs substrate were removed by first mechanical grinding then the etching of the AlGaAs with an HF solution (d). This process is repeated to finally produce a layered structure shown in (e). Since the size of these layers is so small $(\sim 10 \mu \mathrm{m})$, an alignment method was needed in order to be able to stack every other layer at 0.5 a from the same direction layers above and below it. The Noda group utilized a novel laser diffraction alignment method to do this. They found that if the $1^{\text {st }}$ and $3^{\text {rd }}$ layers were lined up on top of each other, the first order diffraction spots would be at $100 \%$ intensity, but would reduce in intensity as the layers were translated with respect to one another. The first order diffraction spots would completely disappear when the layers were aligned correctly at half a period from each other. Thus, they could stack layers with a width, period, and height of $12 \mu \mathrm{m}, 15.5 \mu \mathrm{m}$, and $1.2 \mu \mathrm{m}$ 
respectively, which is not expected to produce a PBG. They proposed that in order to produce a PBG at the optical fiber communication region $(\sim 1.55 \mu \mathrm{m})$, layers of width, period, and depth of $0.16 \mu \mathrm{m}, 0.62 \mu \mathrm{m}$, and $0.19 \mu \mathrm{m}$ respectively would be needed. One of the problems with this method at those sizes is the ability to bond the consecutive layers successfully. Another setback in the procedure was that in order to produce the desired intensity decrease of the first order spots for the correct alignment, a laser was needed with a wavelength close to the distance between the layers. For this reason the team needed to use precision equipment and an infra-red laser-viewing system. Also the precision of the alignment is difficult for smaller sample sizes, but a piezo-actuated alignment system with a resolution limit of $\sim 10-20 \mathrm{~nm}$ is proposed to eliminate that problem.

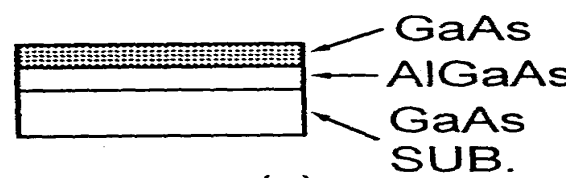

(a)

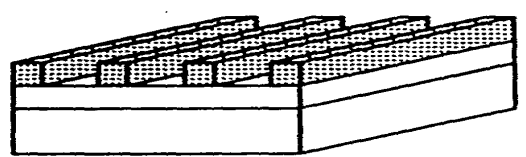

(b)

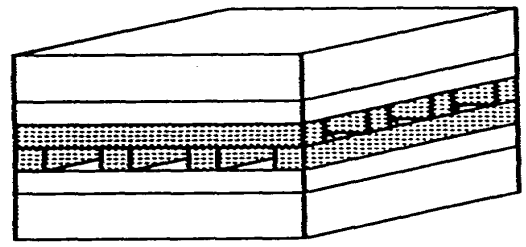

(c)

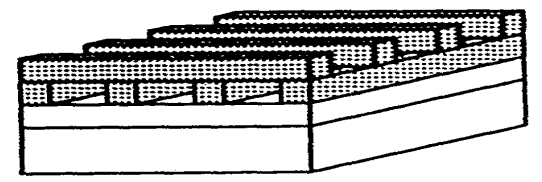

(d)

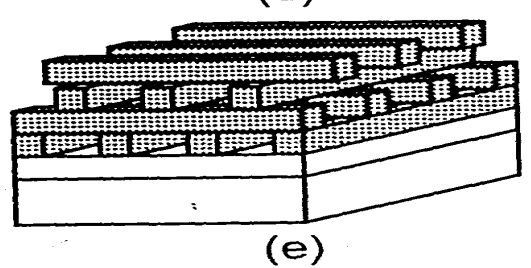

Figure 2.16 - Process steps in creating a four-layer PBG structure in the near infrared regime $^{25}$ 
Most of these problems were solved by the Noda group in $1999^{30}$. They succeeded in using the same process to create a structure with bars of width, period, and depth of 1,4 , and $1.2 \mu \mathrm{m}$ respectively. This structure did produce a complete 3D band gap for frequencies from 5 to $10 \mu \mathrm{m}$ wavelengths. A similar alignment procedure was used to create the structure, but the precision needed to align one-micron bars required the group to develop a slightly different strategy. They fabricated an alignment region next to the active pattern with a larger periodicity that correlated exactly to the smaller region. The large region could be aligned with the laser, and the pattern would follow on a smaller scale such that the first and third layers were displaced by $0.5 \mathrm{a}$. A picture of the structure along with its experimental transmission data is shown in figures 2.17 and 2.18 .

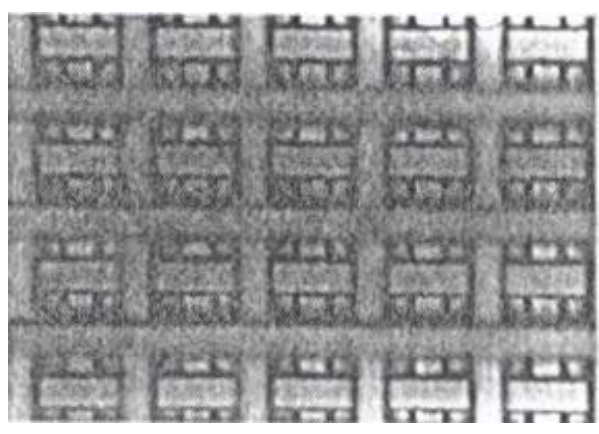

Figure 2.17 - A four-layer structure of GaAs bars produced by lithography and laser alignment methods ${ }^{30}$

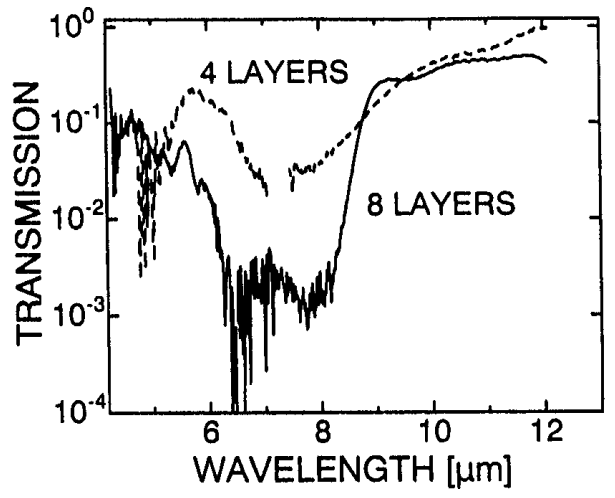

Figure 2.18 - The transmission spectrum for that structure and an eight-layer structure ${ }^{30}$ 
A similar procedure was used by S. Y. Lin et al. at Sandia National Laboratories to fabricate the interconnecting bar samples with semiconductor processing techniques in $1998^{31}$. The layered samples were made by first depositing a layer of $\mathrm{SiO}_{2}$, which was etched with trenches to be filled by polycrystalline silicon. The layer was made flat through a chemical mechanical polishing process and the procedure was repeated, stacking and etching the layers of silicon in the previously explained bar pattern. Finally, the $\mathrm{SiO}_{2}$ was etched away in HF, leaving behind only the layered polycrystalline silicon structure. The final structures were extremely uniform and defect free, but the $5 \mu \mathrm{m}$ bar periodicity was still much too large for optical applications.

In 1996 E. Ozbay introduced several new techniques and procedures for the fabrication and analysis of PBG structures. He first demonstrated a new measurement setup that analyzed transmission and reflection at the same time. The measurement of the previous structures made in the microwave regions all produced nearly $100 \%$ reflection, which is important to the waveguide applications. He also introduced a structure that contained a PBG in the far infrared frequencies. It was etched from a $100 \mu \mathrm{m}$ thick silicon wafer in a similar manner as the silicon wafer used in reference 23 . The bars were $50 \mu \mathrm{m}$ wide with a spacing of $185 \mu \mathrm{m}$, and the structure produced a gap between 378 and $518 \mathrm{GHz}$. He also introduced yet another method for creating the layered PBG structure ${ }^{26}$. Using a high-power carbon dioxide laser to micromachine alumina wafers (refractive index $\sim 2.86$ at $100 \mathrm{GHz}$ ) into $460 \mu \mathrm{m}$ stripes separated by $460 \mu \mathrm{m}$ gaps. The experimental results again correlated to theory, and a gap was seen for frequencies between 81 and $104 \mathrm{GHz}$. Defect structures in the alumina bar design were analyzed and the results were similar to the relationship determined in reference 7 .

A newer method of fabricating the layered structure was introduced by A. Feigel et al. in $2000^{32}$. They utilized photosensitive chalcogenide glasses using a vapor deposition method and direct holographic writing to produce consistent, well aligned samples. As-Se or As-Se-Te glasses were used, which have considerably high refractive indexes ranging from 2.5 to 3 and can also be altered by electromagnetic radiation and etched like a photoresist material. The glasses are also transparent in the infrared from $800 \mathrm{~nm}$ to $12 \mu \mathrm{m}$. The procedural steps to making the PBG structure are depicted in figure 2.19. 


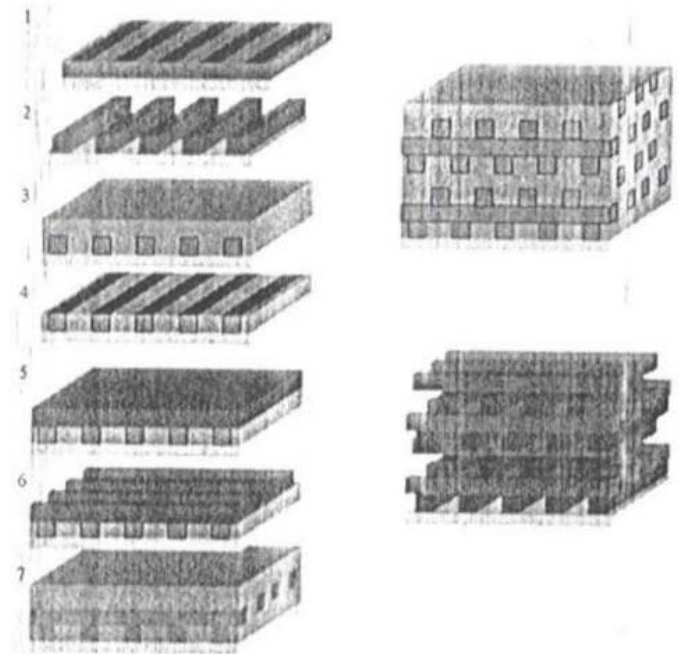

Figure 2.19 - Procedure used to make a layered structure of photosensitive chalcogenide glass $^{32}$

First, the correct ratio of each glass component was evaporated in a vacuum and deposited on a substrate. A holographic grating was written on the layer with a Krypton laser using a two-beam interference setup. The sample was then dipped into a selective developer to create the first layer grating. Steps 3 and 4 above demonstrate that a photoresist was spun into the grooves and etched until it created a planar surface with the glass bars. Then in step 5 , another layer of the chalcogenide glass is deposited, exposed and developed to create a second layer. This process is continued until a satisfactory number of layers have been created. The half-period shift between the $1^{\text {st }}$ and $3^{\text {rd }}$ layers of the structure is realized by using the diffraction patterns similarly to references 30 and 32 .

\section{Colloidal Systems}

One of the first attempts to utilize charged monodispersed polystyrene microspheres to create a PBG structure was carried out by I. Inanc Tarhan and George H. Watson in $1996^{52}$. This was also the first attempt to create a photonic stop band that operated in the visible wavelengths. They suspended the spheres in water, which allowed them to self-order into a face-centered-cubic lattice, but the index of refraction contrast of the spheres was only 1.20, which was not sufficient to create a complete PBG. They utilized Kossel lines to 
characterize the different samples with different lattice parameters, which were easily manipulated with different sized polystyrene spheres. The result of the analysis was that the structure produced a band gap for different directions, but not for all directions at once. Their work does, however, present a method to determine if structure produces a photonic band gap through Kossel line analysis.

This novel idea was used by Wijnhoven and $\operatorname{Vos}^{53}$ to create a photonic band structure (PBS) made of titania at the visible wavelengths. They used monodispersed polystyrene spheres with radii between 180 and $1460 \mathrm{~nm}$ and centrifuged the solution to force the sedimentation of the spheres in glass capillaries. The water surrounding the spheres was evaporated slowly and titania was infiltrated into the network through a precipitation from a liquid-phase chemical reaction. The precursor tetrapropoxy-titanate (TPT) in ethanol penetrated the spheres by capillary action, and was then exposed to the atmosphere to react to form titania. The formed titania was not extremely dense, therefore the procedure had to be repeated up to eight times to produce a connected network. The polystyrene spheres were then slowly burned out at $450^{\circ} \mathrm{C}$ to leave behind an inverse $\mathrm{FCC}$ structure of titania with air "atoms". The titania was characterized to be the anatase phase with a refractive index of $\sim 2.5$. This method produced a structure that contained several defects but if perfected could provide a good photonic band structure at optical wavelengths.

Subramania et al. ${ }^{54}$ formed the titania/polystyrene solution in a one step process in which the polystyrene spheres ordered in the presence of the titania. He used a nanocrystalline titania suspension containing monodispersed polystyrene spheres. A few drops of the slurry were spread on a glass substrate and allowed to dry slowly in a humiditycontrolled environment, which ordered into several layers of the microspheres in a FCC configuration. The sample was then pressed in a cold isostatic press to improve the green density and reduce stress cracks during heat treatment. This caused the c-axis of the sample to contract as the other two of the directions were constrained by the glass substrate during isostatic compression, leading to the slight distortion of the structure. The polystyrene spheres were slowly burned out, leaving the inverse FCC titania network behind. This method produced a better short and long-range order of the structure than the previous methods. A micrograph of the fired sample is shown in figure 2.20. Four different sized 
spheres were used in making the samples and the reflectance spectra for each size and the correlating sphere diameter for each peak is shown in figures 2.21 and 2.22. Each reflectance peak shown in the figure correlates well with the size of the polystyrene spheres used to make the respective sample. Although this procedure produced better, more defect free samples than the previous method, there was still a considerable amount of cracking in the final structures.

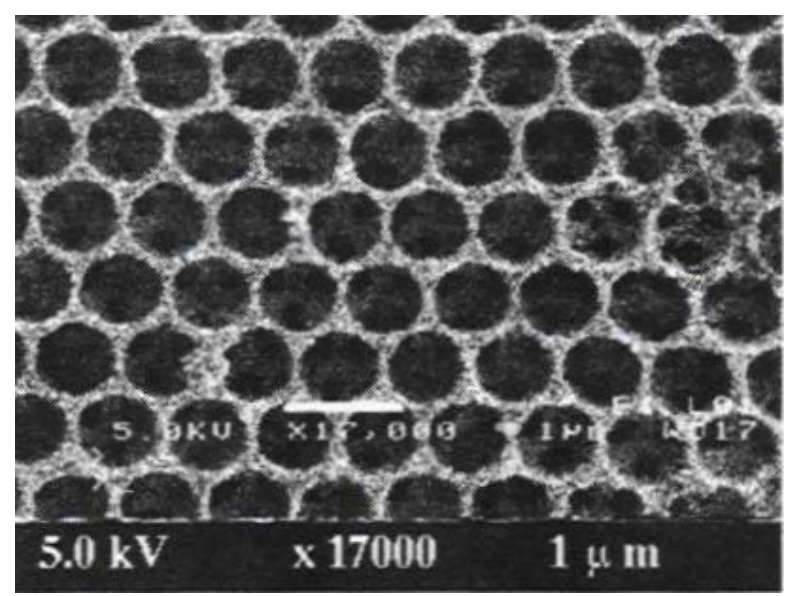

Figure 2.20 - Inverse FCC titania network made using $556 \mathrm{~nm}$ polystyrene spheres with excellent order ${ }^{54}$

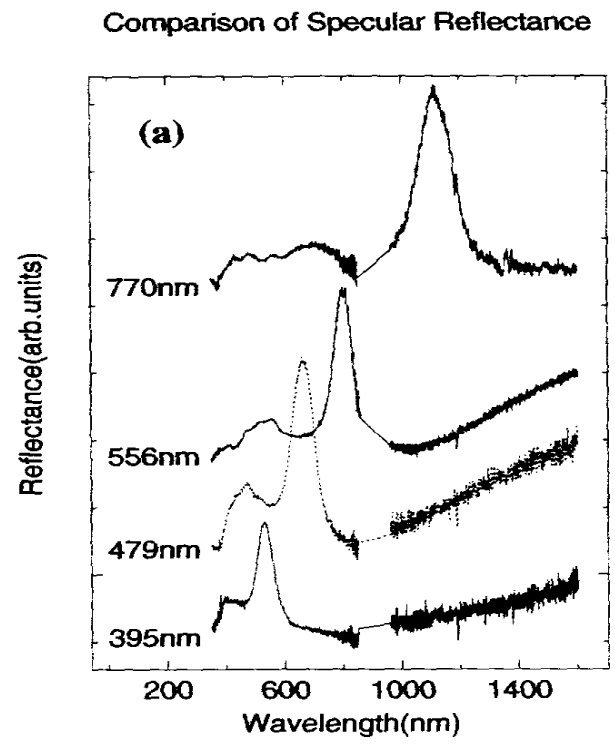

Figure 2.21 - The reflectance spectra for each size of air "atom" in the interconnecting titania inverse FCC matrix ${ }^{54}$ 


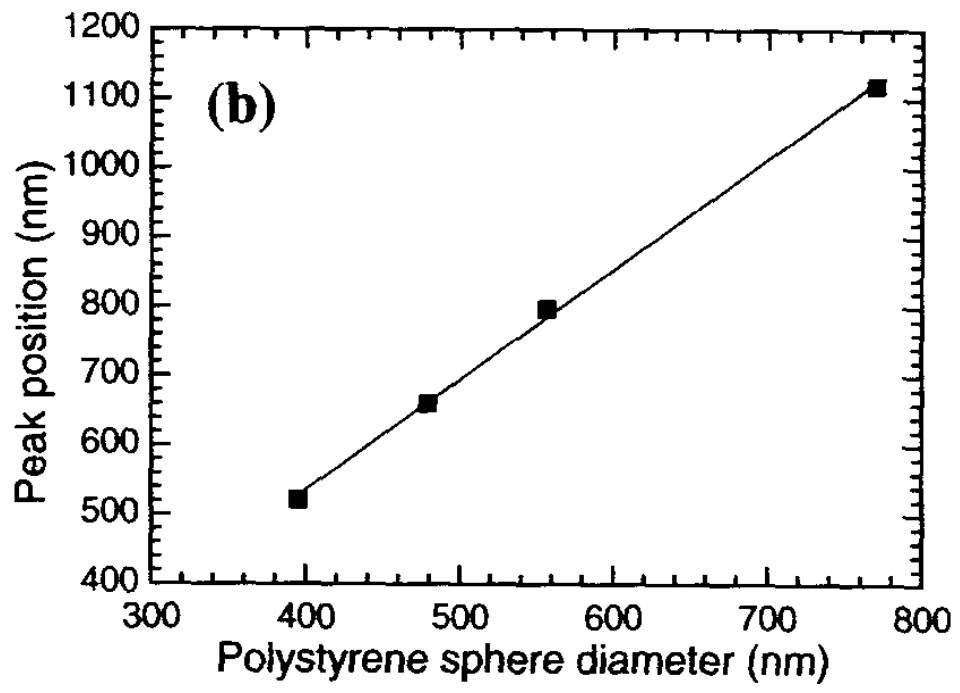

Figure 2.22 - The correlating linear relationship between peak position and sphere diameter ${ }^{54}$

This problem was investigated by H.-B. Sun et al. ${ }^{58}$ in 2000 . The method for producing a defect free assembly of monospheres from a colloid solution was reviewed. The difference introduced into the procedure was the extremely slow quasi-equilibrium evaporation technique. Moisture was removed from the solution in a controlled humidity environment that allowed the water to evaporate extremely slowly. The result was a better short and long-range order in the final sample.

J. Ballato et al. used silica nanospheres (refractive index $\sim 1.446$ ) instead of polystyrene to create a PBG structure at the optical wavelengths ${ }^{55}$. The spheres were allowed to settle under undisturbed conditions in one case, and in another case were centrifuged at $1800 \mathrm{rpm}$. The resulting structures and their respective transmission spectra are shown in figure 2.23. Silica is transparent in the visible range, which suggests that the large transmission decrease that occurred in each case is not related to absorption of the material. The disordered, centrifuged samples created a much larger band gap. Ballato compared this result to x-ray diffraction peaks of amorphous materials, which are wider for materials with no long-range order. 

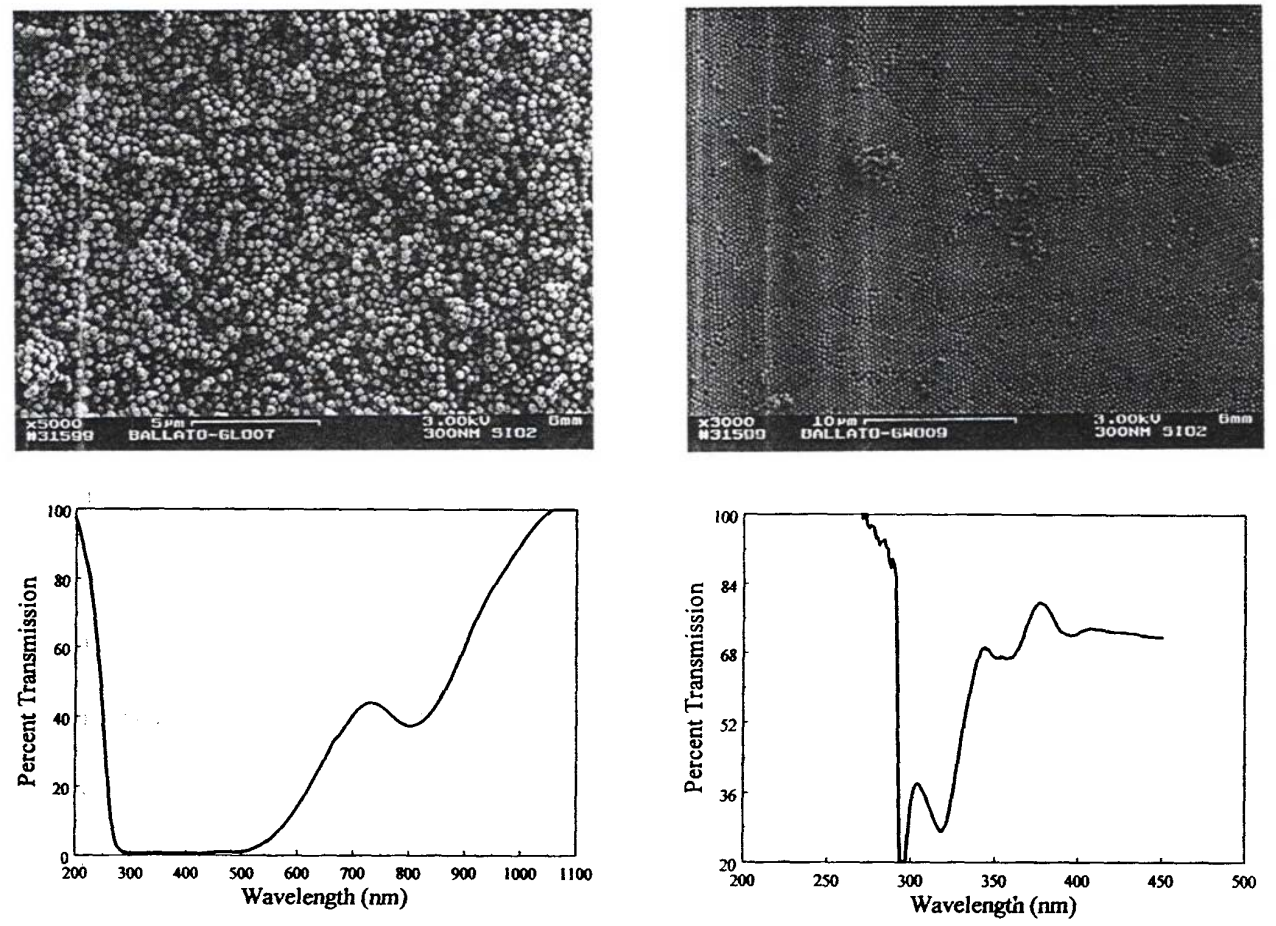

Figure 2.23 - Left side corresponds to silica spheres that were centrifuged at $1800 \mathrm{rpm}$ and the right side corresponds to spheres allowed to sediment undisturbed ${ }^{55}$

Younan Xia et al. used a different procedure to create this same PBG structure from polystyrene spheres with grain sizes larger than one square $\mathrm{cm}^{59}$. A picture that explains the procedure is shown in figure 2.24. They used a method of confined assembly. A cell was constructed from two glass substrates. A square frame was patterned with small channels meant to capture the polystyrene balls and allow the solvent to flow out. They could vary the thickness of the patterned film from 0.5 to $100 \mu \mathrm{m}$, which allowed for the versatility in fabricating samples anywhere from $50 \mathrm{~nm}$ to $50 \mu \mathrm{m}$. A small hole was etched into the top substrate and a glass tube ( $\sim 6 \mathrm{~mm}$ in diameter) was attached with an epoxy. When a solution of nano particles was introduced into the cell, a positive pressure was applied to the tube in order to force the solvent out and the system was constantly sonicated throughout the procedure. The final result was a crystalline structure that formed at the bottom of the cell with very few defects and a large grain size. The group has demonstrated good SEM images of each size of nano-sphere that displayed the excellent order and large grain sizes. 


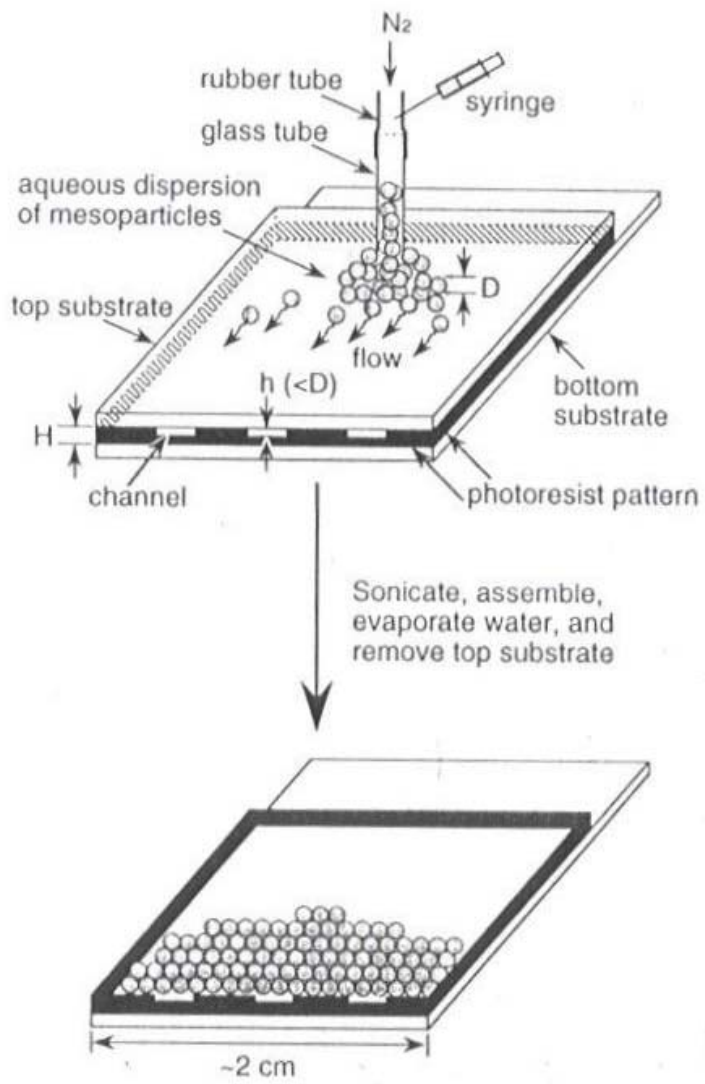

Figure 2.24 - Schematic drawing of the procedure used to order polystyrene spheres over extremely large grain sizes ${ }^{59}$

Y. A. Vlasov et al. analyzed single domains of colloidal photonic crystals in $2000^{60}$. They observed reflection patterns in single grains and also infiltrated the titania structure with CdSe semiconductor quantum dots to provide a method of analyzing the PBG by looking at the spontaneous emission plots. An inverted microscope with a low numerical aperture was used to limit the collection angle to a small cone around the normal incident light. With this setup they could select single crystal domains for analysis, and a CCD array was used to detect the intensity of spontaneous emission. They made measurements on a grain with a (1 111 ) orientation and the reflectance and emission plots from the single grain were compared to macroscopic results. The single grain exhibited a wider PBG in both cases, but further studies were needed to examine all other orientations to be able to announce a full omni-directional PBG. 


\section{Applications and Devices}

\section{Optical Waveguides}

The potential of optical circuitry had been discussed before photonic band gap materials were discovered and developed. There are numerous advantages to using light to operate devices which traditionally use electricity, and PBG structures could provide the

means to do so ${ }^{61-72}$. R. D. Meade et al. was the first group to seriously discuss the possibility of a photonic crystal optical waveguide and microcavity in practical applications ${ }^{61}$. They stated that the current distance necessary to curve light by total internal reflection (TIR) with an optical fiber was around $10 \mathrm{~mm}$, which was much too large to practically develop an optoelectronic integrated circuit (OEIC). The group proposed a 2-D periodic structure to be used as a smaller scale waveguide. They modeled the 2-D case and calculated the band structure for a triangular plane lattice of air atoms as shown in figure 2.25. The proposed 2-D structure still relied on TIR for reflection in the third direction. The group suggested that a three-dimensional photonic crystal with a line defect as the waveguided path was desired but too difficult to create.

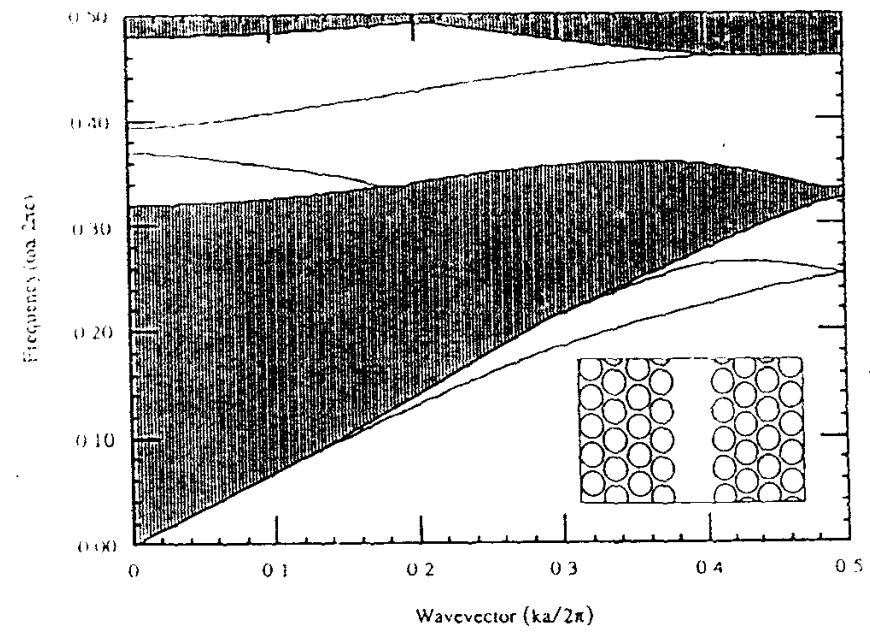

Figure 2.25 - The band structure for a 2-D triangular lattice of air atoms ${ }^{61}$ 
T. A. Birks et al. have been working on producing a photonic crystal fiber that forces electromagnetic radiation to propagate in a defect air cavity in the fiber structure ${ }^{63,64,66,68}$. They created the fiber entirely from fused silica. It was drawn in a manner that left a hexagonal honeycomb pattern of air holes that ran the length of the fiber. The first fiber the group produced contained a silica defect in the center so that the surrounding fiber containing the pattern of air holes had a lower index of refraction. Therefore, this first sample operated completely on total internal reflection, but the photonic band gap properties of the cladding forced the fiber to guide only a single mode of electromagnetic radiation, which the sample could do from $337 \mathrm{~nm}$ to $1550 \mathrm{~nm}$. A schematic drawing of the fiber is shown in figure 2.26. The group eventually used a similar method to draw a photonic crystal fiber that contained an air defect in the center instead of a silica defect by placing a hollow silica fiber in the center. A micrograph of the final cross-section of the drawn fiber is shown in figure 2.27. This new fiber was found to propagate light through photonic effects rather than total internal reflection. The fiber in later experiments only transmitted $35 \%$ of a laser beam incident at one end of a $40 \mathrm{~mm}$ fiber, however most of the losses were attributed to input coupling efficiency. The group observed that several modes of light were transmitted.

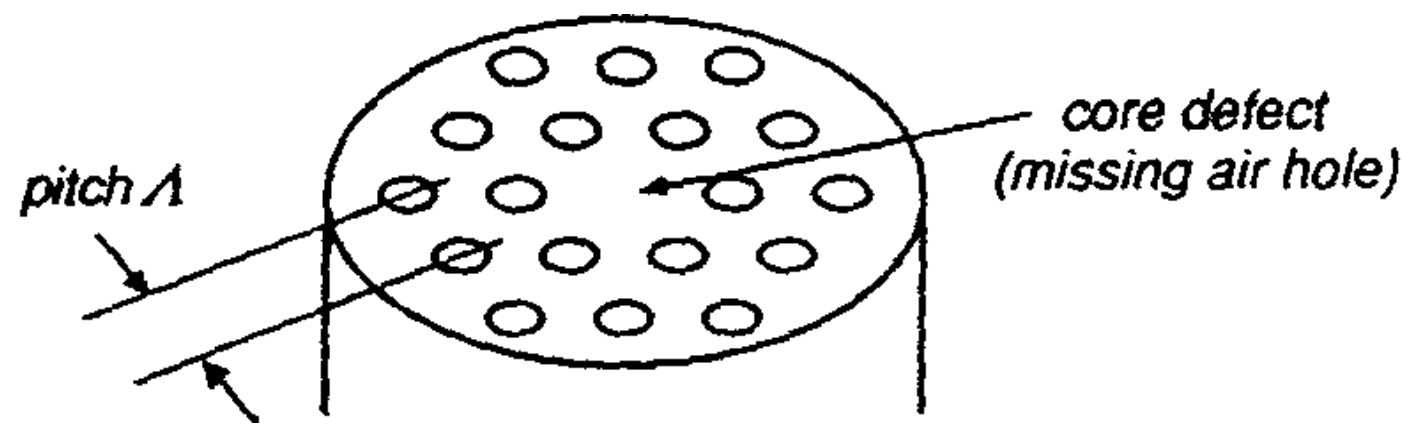

Figure 2.26 - A drawing of a photonic crystal fiber. Its photonic properties are derived from the missing air hole in the center ${ }^{64}$ 


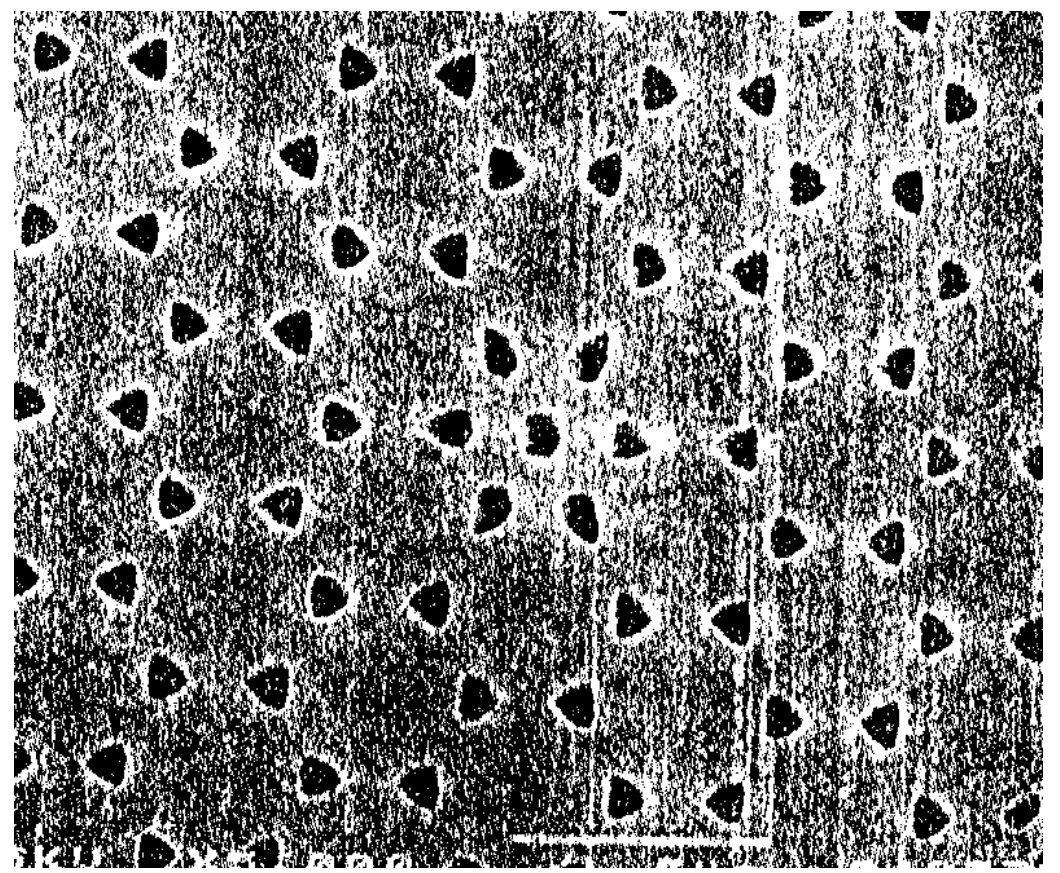

Figure 2.27 - Cross-section of a photonic crystal fiber containing an air defect in the center ${ }^{66}$

S.-Y. Lin et al. was the first to develop a PBG waveguide at the near infrared frequencies that would turn a sharp 90 degree corner at nearly perfect transmission efficiencies $^{65}$. The researchers created the structure with a 2-D square array of alumina rods with a dielectric constant of 8.9 and a radius of one-fifth of the lattice parameter. A line defect was introduced into the structure by removing a row of the rods to provide a propagation path for the determined frequency mode. A schematic diagram of the structure is shown in figure 2.28. The group tested the transmission of the straight PBG waveguide and 2 configurations of waveguides that forced the radiation to turn corners. In this situation, the light propagated through an air defect rather than an absorbing medium, which increased transmission efficiency by a factor of ten. They also demonstrated that light can be turned 90 degrees over the distance of one wavelength, which was the smallest radius achieved in a dielectric waveguide with $100 \%$ transmission. 


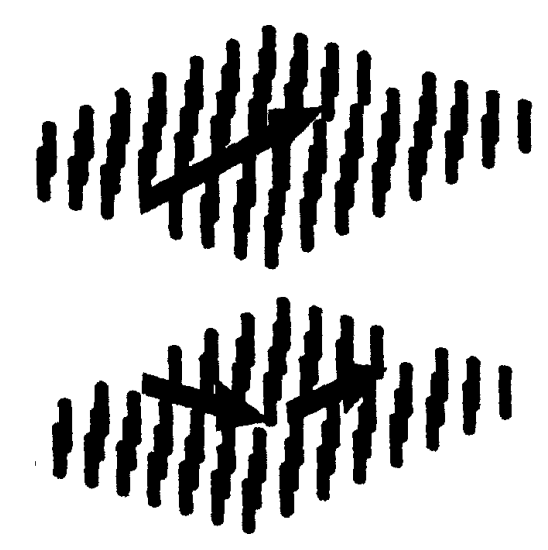

Figure 2.28 - PBG waveguide made of alumina rods that effectively transmits near-infrared frequencies at low loss ${ }^{65}$

The next step in the advance of optical circuits was the ability to cross the optical transmission lines without lasing or distortion losses of the signal. This advance was researched by S. G. Johnson et al. ${ }^{67}$ They modeled a perpendicular waveguide crossing that achieved nearly $100 \%$ throughput with cross talk on the order of $10^{-9}$. The conditions for this optimal performance are outlined as follows:

- Each waveguide must have a mirror symmetry plane through its axis and perpendicular to the other waveguide and have a single guided mode in the frequency range of interest. This mode will be either even or odd with respect to the mirror plane.

- The center of the intersection must be occupied by a resonant cavity that is symmetric with respect to the mirror planes of both waveguides.

- Two resonant modes must exist in the cavity, each of which is even with respect to one waveguide's mirror plane and odd with respect to the other. These modes should be the only resonant modes in the frequency range of interest.

For their first set of experiments, the group used PBG waveguides of four different structural variations. The throughput and crosstalk plots are shown in figure 2.29. As shown in the diagrams, the introduction of the air holes increased the throughput from $75 \%$ to $90 \%$ and decreased the crosstalk from 7 to 0.08 . 


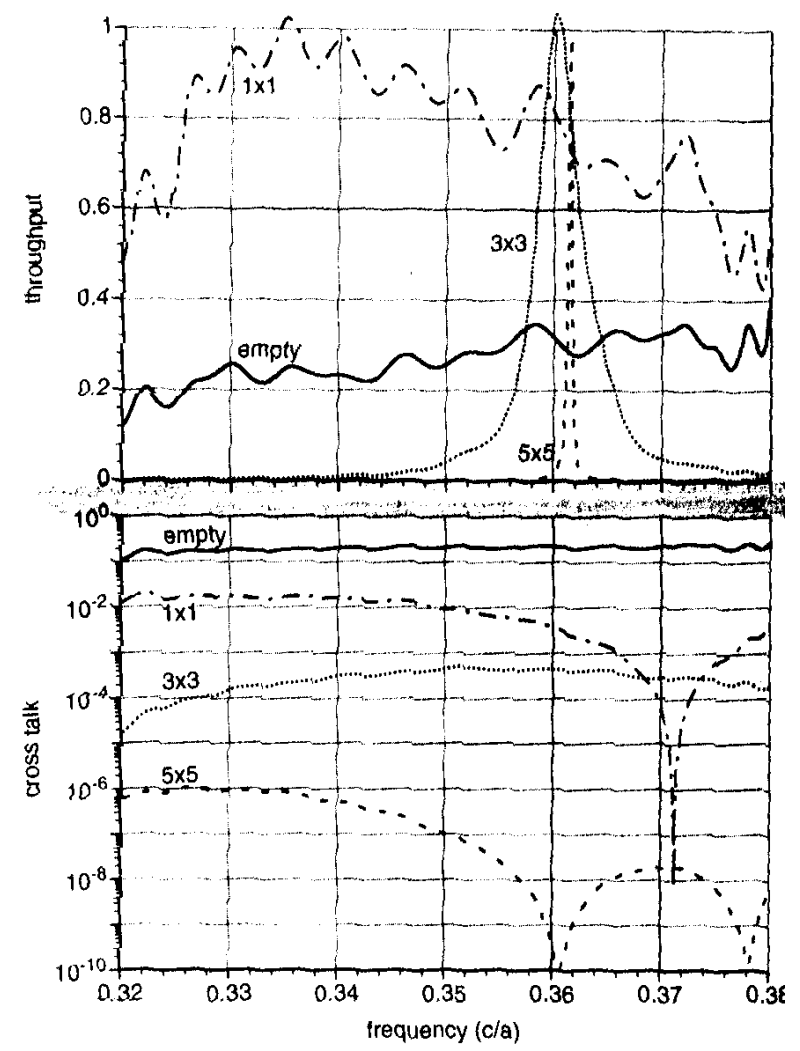

Figure 2.29 - Throughput and crosstalk diagrams corresponding to the amount of photonic crystal within the waveguide intersection ${ }^{67}$

C. Jin et al. proposed a photonic crystal fiber (PCF) utilizing a photonic quasi-crystal rather than a long-range ordered structure ${ }^{70}$. The group created a 2-D octagonal quasicrystal constructed with a $23 \times 23$ array of alumina cylinders embedded in a Styrofoam template as shown schematically in figure 2.30. They found that that the straight and zigzag waveguides that had been tested exhibited very high transmission efficiency for wavelengths within the band gap. The actual band gap of the tested structure fell between 8.9 and $10.9 \mathrm{GHz}$. They also found that the incident angle of the radiation on the structure had little effect on the percentage of transmitted frequencies. The transmission plot demonstrating the structure's PBG is shown in figure 2.31 . 


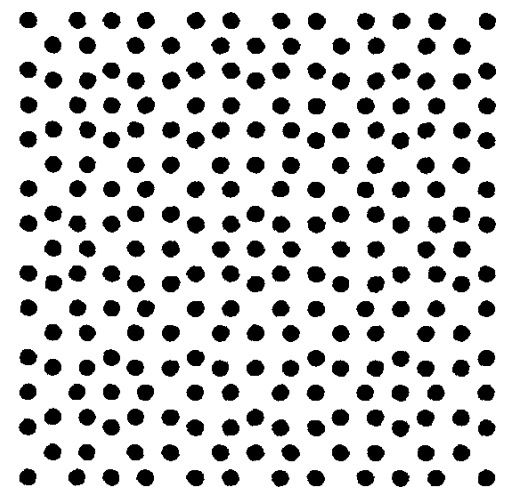

Figure 2.30 - Schematic top view of the alumina rod structure ${ }^{70}$

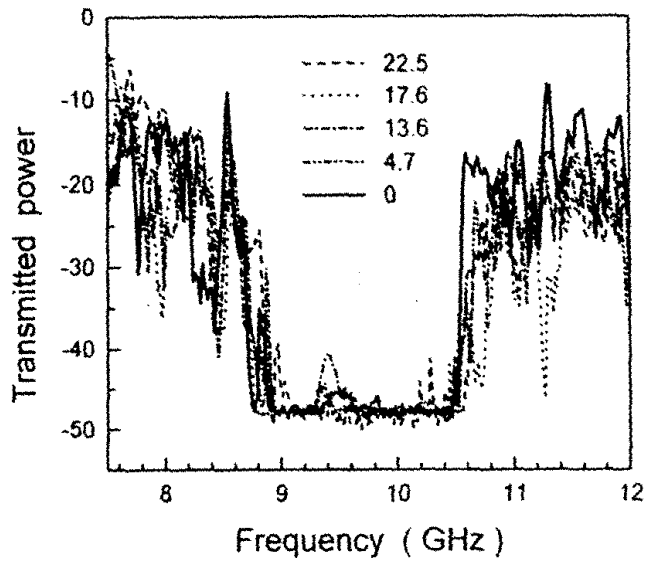

Figure 2.31 - Transmitted signal of the waveguided structure in arbitrary units. The different curves represent the different paths shown and numbered next to the transmission diagram ${ }^{70}$

A. Chutinan and S. Noda proposed the first three dimensional photonic crystal that could be used in a practical optical circuit ${ }^{72}$ as shown in figure 2.32 . The group used the previously described layered bar method to produce the waveguide. They simply removed one of the bars in the layered structure to allow a guided mode to propagate along that defect path. In order to change the direction of the light, the group first tried to remove portions of the bars in the same layer 90 degrees with respect to the original defect channel. This proved to be inefficient because of poor mode coupling between the two states. They did, however, succeed by removing a second channel perpendicular to the first, but in an adjacent layer. The radiation transmitted smoothly between the layers with an efficiency of over $95 \%$, which 
was considerably better than conventional techniques with the same radius of curvature. The group then calculated the necessary minimum number of layers to efficiently confine the light to be twelve: five below, two defect layers, and five above. As the fabrication technology progresses, this process can be used to develop small optic circuits with low losses.

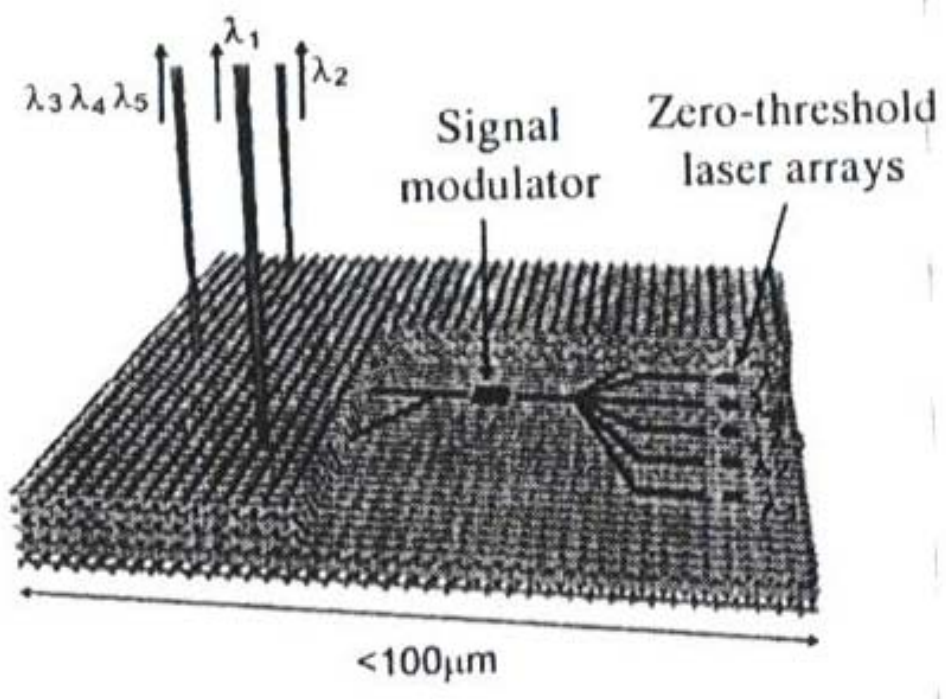

Figure 2.32 - a hypothetical schematic of optical circuitry containing zero-threshold laser arrays, signal filters and optical waveguides ${ }^{72}$

The same group used the same production methods to fabricate a waveguide structure small enough to operate at communication wavelengths around $1300 \mathrm{~nm}^{73}$. The SEM image of the final waveguide structure with a 90 degree bend is shown in figure 2.33 on the following page. The sample was the first 3-D waveguide created with a bend that was able to transmit light. It had a transmission of greater than $95 \%$ over a wide frequency range from 0.553 to $0.605[\mathrm{c} / \mathrm{a}]$.

Masatoshi Tokushima et al. have fabricated a 2-D waveguide that successfully propagates wavelengths around $1550 \mathrm{~nm}$ around a sharp bend of 120 degrees $^{74}$. An SEM image of the sample is shown in figure 2.34. They started with a silicon-on-insulator wafer with 2 microns of silicon on top of 2 microns of $\mathrm{SiO}_{2}$. Another thin $150 \mathrm{~nm}$ layer of $\mathrm{SiO}_{2}$ was formed on the top of the silicon through oxidation. Triangular lattice holes with a pitch 
of $800 \mathrm{~nm}$, as shown in figure 2.34, were patterned and etched on the $\mathrm{SiO}_{2}$ layer with standard photolithography techniques. The layers were then successively etched to create the final pattern including a waveguide. Each edge of the waveguide was coupled to a taperedhemispherical-end fiber, which were used for the input and detection testing of the waveguide. It was ensured that no EM radiation could couple between the fibers, except through the guided path. The measurements resulted in a transmitted signal with a FWHM of around $4 \mu \mathrm{m}$.

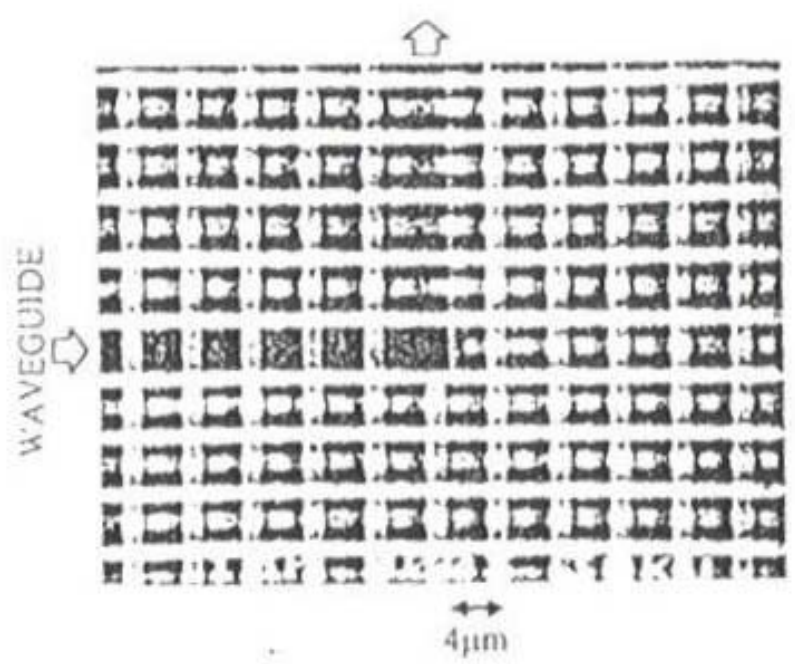

Figure 2.33 - SEM image of a waveguide structure with a bar period of $700 \mathrm{~nm}$ containing a defect meant to propagate light through a 90 degree corner $^{73}$

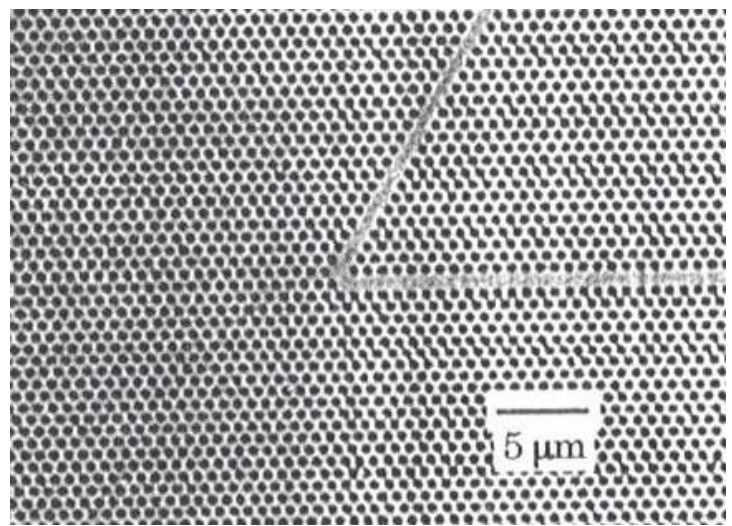

Figure 2.34 - An SEM image of a 2-D sample with a waveguide path undergoing a 120degree bend $d^{2.114}$ 


\section{Lasers and light emitting devices}

Ever since the original discovery of the properties of photonic band gap materials, there has been considerable research directed towards the development of smaller, more efficient LED's and lasers ${ }^{76-89}$.

The first discoveries dealing with laser emission at zero threshold were centered around microcavities. The microcavities that were developed inhibited spontaneous emission and produced high stimulated-emission gain at low excitation levels. This was done at an extremely small scale ${ }^{91,76-79}$. Lasers had been proven to work at an excitation threshold of $\sim 50 \mathrm{pJ}$ when excited by a femtosecond source. The addition of a 2-dimensional photonic crystal to the microcavity proved to increase the gain of the laser even further ${ }^{84}$. In 1999, R. $\mathrm{K}$. Lee et al. showed that the 2-D crystal doubled the gain of the microcavity laser by the inhibition of spontaneous emission in the lasing plane.

The idea of the microcavity spawned the photonic band edge laser, ${ }^{80,92}$ which made use of PBG properties to increase the gain of the laser four-fold in vertical cavity surfaceemitting lasers (VCSELs). One-dimensional periodic layers of gain media and dielectric material of 20 periods were tested and the result was the amplification of the frequencies at the low end of the prohibited gap. This was caused by the reflection inside the structure between the dielectric layers (inside the gain media) that stimulated photons at energies just outside the band gap. This extra time that the photons existed inside the gain media contributed to the total increase in the gain when the frequency outside the band gap is finally transmitted.

Y. A. Vlasov and his research group in Strasbourg Cedex, France, were the first to observe the effects of a pumped semiconductor infiltrated within a PBG matrix ${ }^{81}$. They used synthetic opal structures fabricated by a sol-gel synthesis of a monodispersed suspension of silica spheres, which they then infiltrated with vapor flows of cadmium and sulfide. The result was a photonic crystal with a pseudo-gap surrounding nanocrystals of the semiconductor $\mathrm{CdS}$. The crystal was then pumped by a XeCl excimer laser. The team found that the interference of the photonic crystal resulted in enhancement of the long-wavelength portion of the gain at the low-frequency edge of the photonic stop band. The lasing action of 
the crystal was observed to increase exponentially with threshold pumping and it didn't require any external mirrors or cavities.

M. Meier et al. at Bell Laboratories in New Jersey experimented with the application of organic gain media into a periodic structure of small holes etched into silica ${ }^{83}$. When photoexcited with a pulsed nitrogen laser of $337 \mathrm{~nm}$, the structure emitted two sharp peaks: a transverse electric (TE) mode at $580 \mathrm{~nm}$ and a transverse magnetic (TM) mode at $596 \mathrm{~nm}$. The structure was found to only have a pseudo photonic band gap, but it was still proposed that the prohibition of photons in certain directions gave rise to the laser oscillations.

Several papers have been published that discuss two-dimensional PBG defect lasers and surface-emitting lasers constructed by several different production methods ${ }^{85-90}$. The production techniques have ranged from photolithography and etching methods to elastomeric stamping of a sol-gel nanoparticle glass to forming an organic photonic crystal by replica molding. Each of these procedures has been used to produce a triagonal or hexagonal array of air holes in a dielectric gain media. In each case, the efficiency of the device was drastically increased by the 2-dimensional PBG structure in place. The output spectrum generally showed a large peak at the band gap and the device experienced low threshold pumping values. These developments were predicted to lead to new laser and light-emitting diode devices that require less power, emit less heat, and provide more light that previous designs.

Mirrorless lasing was achieved in 2000 by Peidong Yang et al. with mesostructured waveguides ${ }^{93}$. They used a micromolding in capillaries and microtransfer molding techniques to fabricate mesoporous silica bars doped with Rhodamine 6G. The line patterns were pumped by a $532 \mathrm{~nm}$ frequency-doubled neodymium-yttrium-aluminum-garnet laser, and the photoluminescence spectrum was detected from the ends of the doped bars. The experimental setup is shown in figure 86. A low threshold pumping energy of $10 \mathrm{~kW} / \mathrm{cm}^{2}$ was needed to produce amplified spontaneous emission from the ends of the patterned bars, which did not utilize a PBG effect. The group used a thermoelectrically cooled CCD detector to analyze the photoluminescence spectra. They found that for higher pumping intensities, the photoluminescence intensity grew superlinearly and the spectral width decreased to a minimum of $7 \mathrm{~nm}$. The group concluded that the study could be furthered by 
examining different host materials for the laser dye or by experimenting with different laser dyes to look for different optical results. Even though the experiment did not make use of a PBG effect, the process could be used in a PBG structure to amplify the observed photoluminescence.

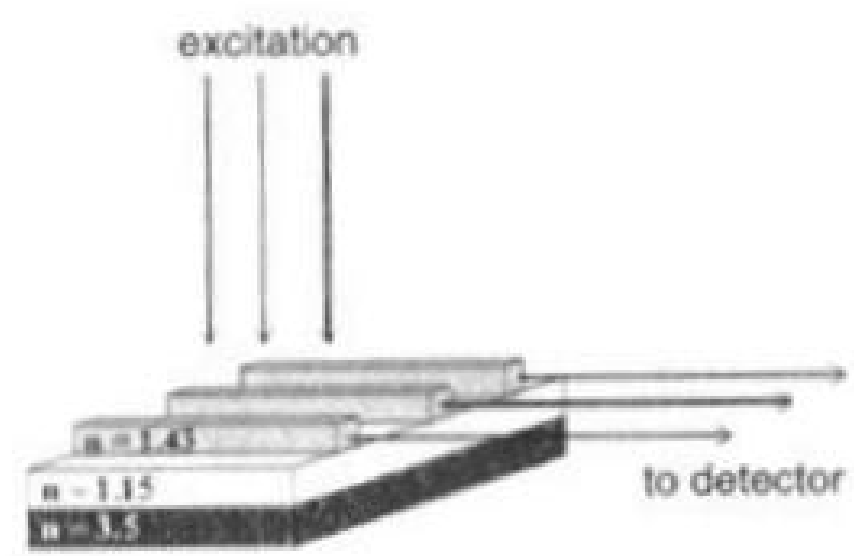

Figure 2.35- Experimental setup demonstrating mirrorless lasing ${ }^{93}$

\section{Sensors}

Photonic band gap materials have also proven useful in the area of detectors. ${ }^{94-96} \mathrm{~A}$ detector placed in a defect mode of a three-dimensional PBG structure will become a resonant cavity enhanced photodetector. The electromagnetic radiation of a certain wavelength can be separated in this manner and can arrive at the detector without external noise. Optical temperature sensors have also been proposed by John Ballato at Clemson. $\mathrm{He}$ used the silica structure discussed previously to infiltrate with 1-methylnaphthalene (MN), a material that has a negative index of refraction dependence with temperature. As the temperature of the photonic crystal changes, so does the transmission through it as a result of a change in index of refraction contrast between the $\mathrm{MN}$ and the silica. The dependence is fairly linear, and the sensor is projected to have several applications from optical switching to thermometers. Similar sensor mechanisms are also possible by utilizing the same transmission dependence on certain properties as suggested by A. Z. Genack and N. Garcia ${ }^{97}$. They mention electronic sensing applications as a result of displacement of a piezoelectric 
matrix that could alter the periodicity of the PBG, and therefore, the transmission. The authors also discuss the possibilities of frequency identification because of the ability of a photonic crystal to identify a frequency of a tunable source from its transmitted characteristic peaks.

The vast application possibilities for photonic band gap materials cannot be completely covered in this proposal. As the current major areas of interest have been discussed, a few remaining applications will be simply mentioned ${ }^{98-104}$. PBG's have been used as substrates for microwave antennas to improve their efficiency. Without a PBG substrate, much of the power of the antenna is trapped within the high refractive index substrate because of TIR. The PBG substrate forces the emitted radiation to propagate into the air. Another more obvious application that has been discussed is the use of a metallic/dielectric band gap structure to use as a frequency filter in the infrared frequencies. This could possibly be used in current circuitry to sort signals. An interesting possibility for PBG structures was suggested by D. F. Sievenpiper et al. ${ }^{101}$ They discussed the use of photonic crystals for optical image processing. The structure they developed can perform several Boolean logic functions at one time rather than the less efficient circuitry used today.

\section{Fabrication Methods}

The method in which the PBG structures are created, for the research purposes of this proposal, is called micro transfer molding ( $\mu \mathrm{TM})$. A polydimethylsiloxane (PDMS) elastomer mold was infiltrated with an epoxy to apply to a substrate iteratively to produce a 3-D epoxy bar structure. Some of the first work in this area involved the usage of the PDMS mold as a stamp to create gold patterns on a substrate called micro contact printing $(\mu \mathrm{CP})^{143}$. A. Kumar et al. used a PDMS mold formed from a patterned silicon wafer. They coated the mold with a hexadecanethiol solution, which was then stamped onto a silicon substrate covered by a thin gold layer. The substrate was then etched, leaving behind only the stamped gold regions. This process is shown in figure 2.36 . 


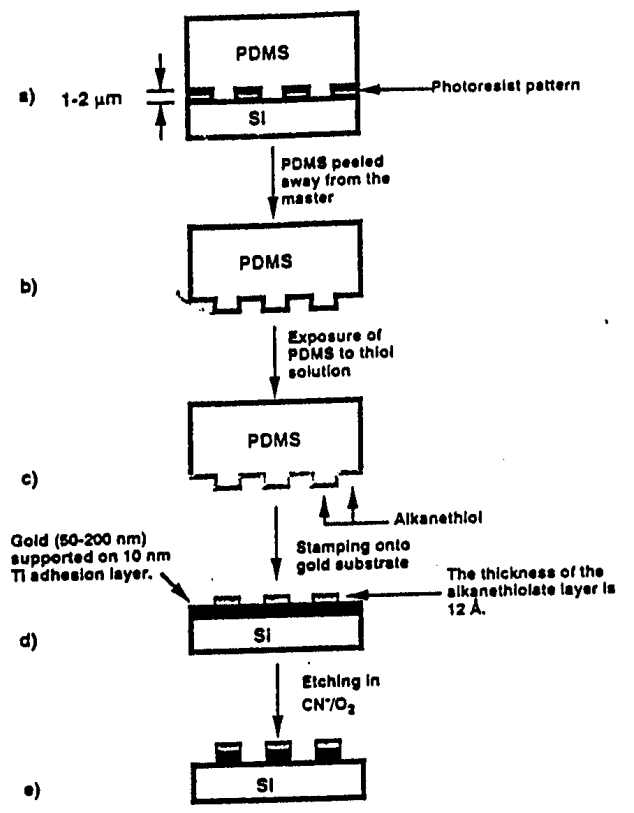

Figure $2.36-\mu \mathrm{CP}$ Process used to stamp patterns onto a gold layer in order to make small features $^{143}$

Feature sizes from 1-100 $\mu \mathrm{m}$ were created using this technique. The advantage of this method over conventional photolithography techniques was the ease and speed at which features could be produced. Multiple impressions could be made by the same mold without additional photolithography steps. The disadvantage of the process was the problem of the sharpness of the desired design. Lines and features may become blurred as the ink is applied unevenly.

From this $\mu \mathrm{CP}$ stamping method, a micro transfer molding ( $\mu \mathrm{TM}$ ) procedure was developed which made the production of three-dimensional structures possible. X.-M. Zhao et. $\mathrm{al}^{145}$ began using the PDMS molds to infiltrate with a liquid precursor by capillary action. The mold could then be placed on a substrate and peeled away after the setting of the precursor, leaving behind only the polymer. This procedure was effective on smooth or rough surfaces, which made it possible to stack layers of certain designs, developing a 3-D structure. This process is shown in figure 2.37. 


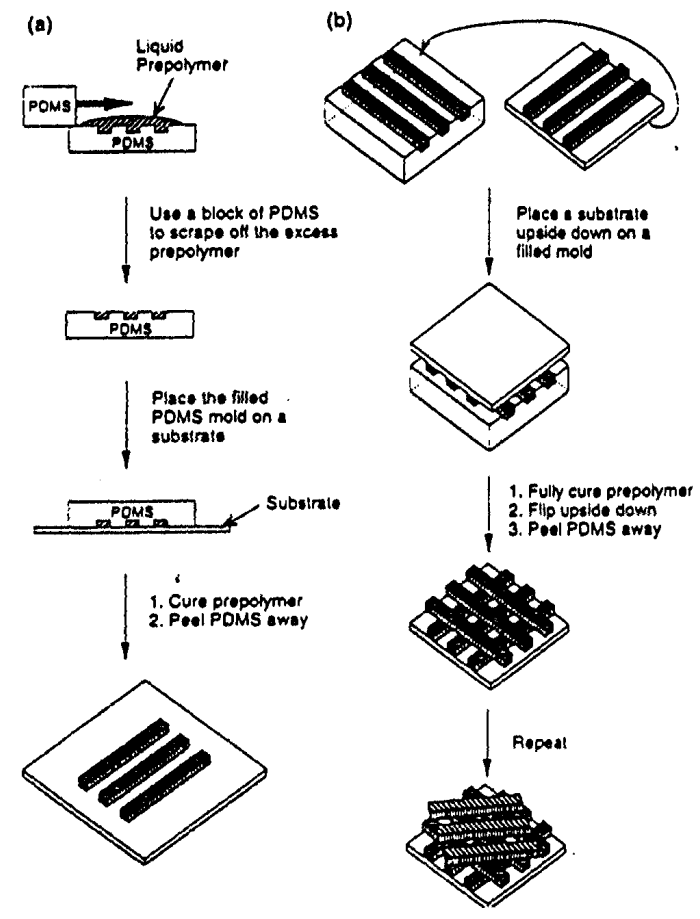

Figure 2.37 - The $\mu \mathrm{TM}$ procedure used to stack epoxy bars, utilizing the PDMS elastomer $\operatorname{mold}^{145}$

The group infiltrated the mold by placing a drop of the prepolymer over the patterned area and removing the excess by blowing nitrogen or using another flat piece of PDMS to scrape it away. The whole process took less than five minutes. As shown in the figure, the method was a simple, efficient method that could be used to build a 3-D PBG structure. A small layer of the polymer was detected on the substrate after application because of poor excess control, but did not appear to affect the $2^{\text {nd }}$ or $3^{\text {rd }}$ layers significantly.

The microtransfer molding techinique was adopted by the Iowa State University research group, who had been developing 3-D PBG structures by using other ceramic processing methods. The team infiltrated the polymer 3-D structure with titania by means of slurry infiltration or sol-gel techniques ${ }^{146}$. This method made possible the production of much smaller photonic crystals than previously could be made. After slurry or sol-gel infiltration and firing, a titania bar structure remained that produced a three-dimensional photonic band gap in the near infrared frequencies. 


\section{Laser Dyes with Scatterers}

Organic dye lasers have been used for many years as a tunable source of high intensity and short wavelength laser luminescence ${ }^{105}$. There are four principal types of organic dye lasers that are characterized by the energy source used to initiate the laser action. They are the flashlamp pumped, nitrogen laser pumped, neodymium laser pumped, and argon ion laser pumped systems. These lasers can be tuned to emit different wavelengths by changing the concentration of the dye.

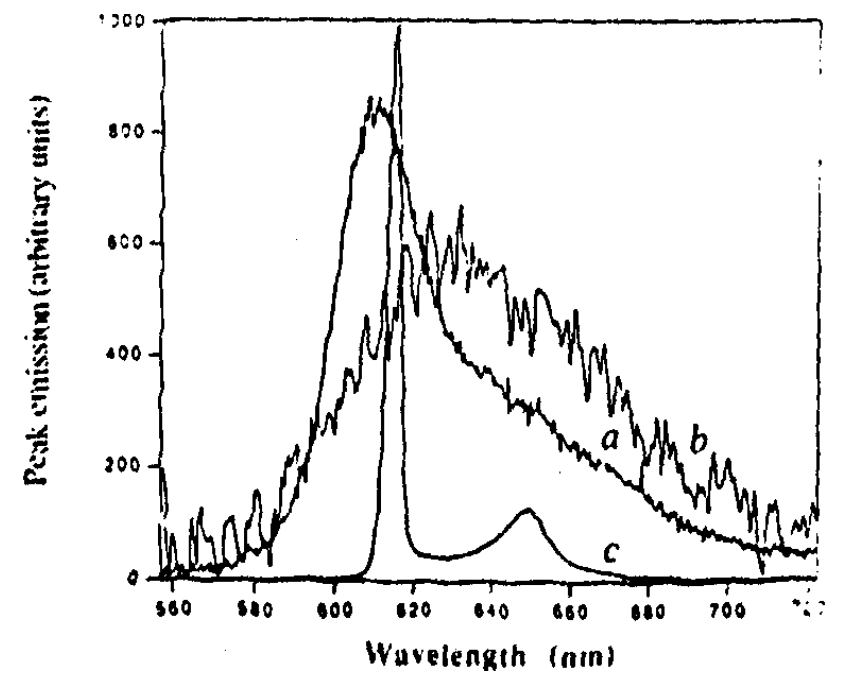

Figure 2.38 - Emission spectrum of a $2.5 \times 10^{-3} \mathrm{M}$ solution of rhodaminne 640 perchlorate in methanol pumped by 3-mJ (7-ns) pulses for (a) and (c), and $2.2 \mu \mathrm{J}$ (7-ns) pulses for (b) at $532 \mathrm{~nm}$. Solutions (b) and (c) also contain $2.8 \times 10^{10} \mathrm{~cm}^{-3} \mathrm{TiO}_{2}$ nanoparticles. The amplitude of (b) is scaled up 10 while the amplitude of (c) is scaled down 20 to fit on the same plot ${ }^{105}$

A recent area of interest in the dye laser field is the introduction of a scattering media within the dye $\mathrm{e}^{108-119}$. Several authors have found that scattering particles dispersed within a polymer gain media will increase intensity while decreasing the threshold and linewidth of the laser-action emission. Figure 2.38 demonstrates the ability to increase intensity and decrease the linewidth of a laser dye by the addition of scattering particles. It was suggested 
that the scattering media introduced into the laser dye would suppress the spontaneous emission and enhance the stimulated emission of the laser, which was a desired effect. The concentration of the laser dye material and scattering media within the dye were found to have a large effect on the luminescence spectrum of the laser. Therefore, the ideal concentrations were investigated and found to be a $2.5 \times 10^{-3} \mathrm{M}$ solution of the dye with a density of $\sim 10^{11}$ scattering nanoparticles per $\mathrm{cm}^{3}$. Scattering particles also reduced the threshold power needed to initiate lasing in each dye laser, which was thought to be a result of an increased laser beam path through the media as the beam is reflected back and forth. This is similar to the band edge laser discussed earlier in this paper, operating on photonic band effects instead of scattering particles.

Opal structures were also infiltrated with laser dyes in an attempt to achieve similar lasing results as the scattering media ${ }^{120-123}$. Silica spheres were generally used to create the structures to be infiltrated with a solution of rhodamine $6 \mathrm{G}$ or other dyes. The tests also resulted in the linewidth narrowing of the spectral emission of the samples when pumped by another laser source. This was also attributed to amplified spontaneous emission (ASE) because of the internal reflection from the opal structure and its PBG effects.

In 1998, E. P. Petrov et al. demonstrated the effect of a PBG structure on the emission of a laser dye $\mathrm{e}^{124}$. They used an artificial opal structure of 200-300 nm silica spheres, which was infiltrated with the laser dye 1,8-naphthoylene-1 ',2`-benzimidazole $(7 \mathrm{H}$ benzimidazo[2,1-a]benz[de]isoquinolin-7-1) (NBIA) in poly methyl methacrylate (PMMA). This was compared to a thick polymer film of the NBIA in PMMA. The opal sample used demonstrated a pronounced stop band in the $500 \mathrm{~nm}$ region, which lies in the fluorescence emission band of NBIA. The sample was excited by a TEA $\mathrm{N}_{2}$ laser with a pulse duration of $500 \mathrm{ps}$ and a wavelength of $337.1 \mathrm{~nm}$. The fluorescence measurements were collected in the direction normal to the surface within a solid angle of $0.09 \pi$. The result of the experiment was that the PBG structure inhibited spontaneous emission at the stop band wavelength and also shifted electron decay times both ahead and behind the control sample decay times. The spontaneous emission plots for both samples are shown in figure 2.39. 


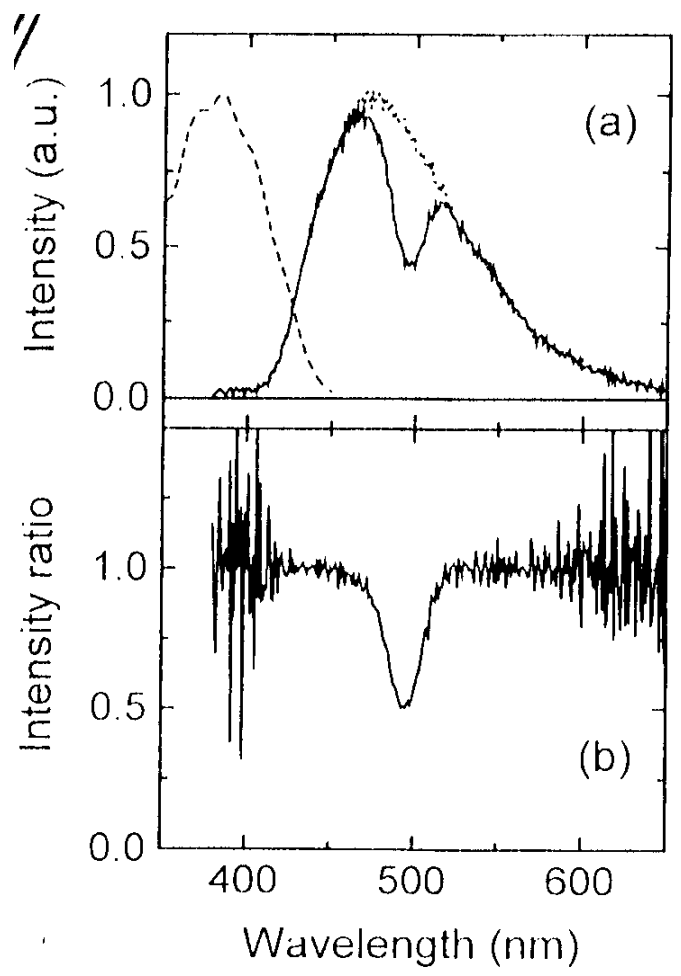

Figure 2.39 - (a) Photoluminescence spectra for $(-)$ the NBIA dye polymer infiltrated into the photonic opal crystal and ( --- ) The control NBIA dye polymer alone. The dashed peak to the left is the pumping laser spectrum (b) The ratio of intensity between PBG infiltration and control samples ${ }^{124}$

\section{PPV Polymer Luminescence}

Poly(p-phenylene vinylene) (PPV) was one of the first polymers to be used as the light emitting layer in a light-emitting diode (LED) The original efficiency measurements of the semi-conducting polymer were around $0.01 \%$ of photons emitted per injected electron ${ }^{125}$. Since the discovery, variables such as setting time, temperature, amount of conjugation of the polymer, addition of an electron transport layer, electrode material selection, and the introduction of disorder into the polymer have been optimized to develop a gain layer with efficiencies around $2 \%$ of photons emitted per injected electron ${ }^{125-142}$. 


\section{CHAPTER 3: EXPERIMENTAL APPROACH}

\section{Mold Fabrication}

Photonic band gap structures can be produced in many ways. Our research is focused on the building of the PBG structure with epoxy bars. The first step in this procedure is creating an epoxy bar structure in which titania can later be infiltrated. This procedure is outlined below step by step and is depicted in figure 3.1 .

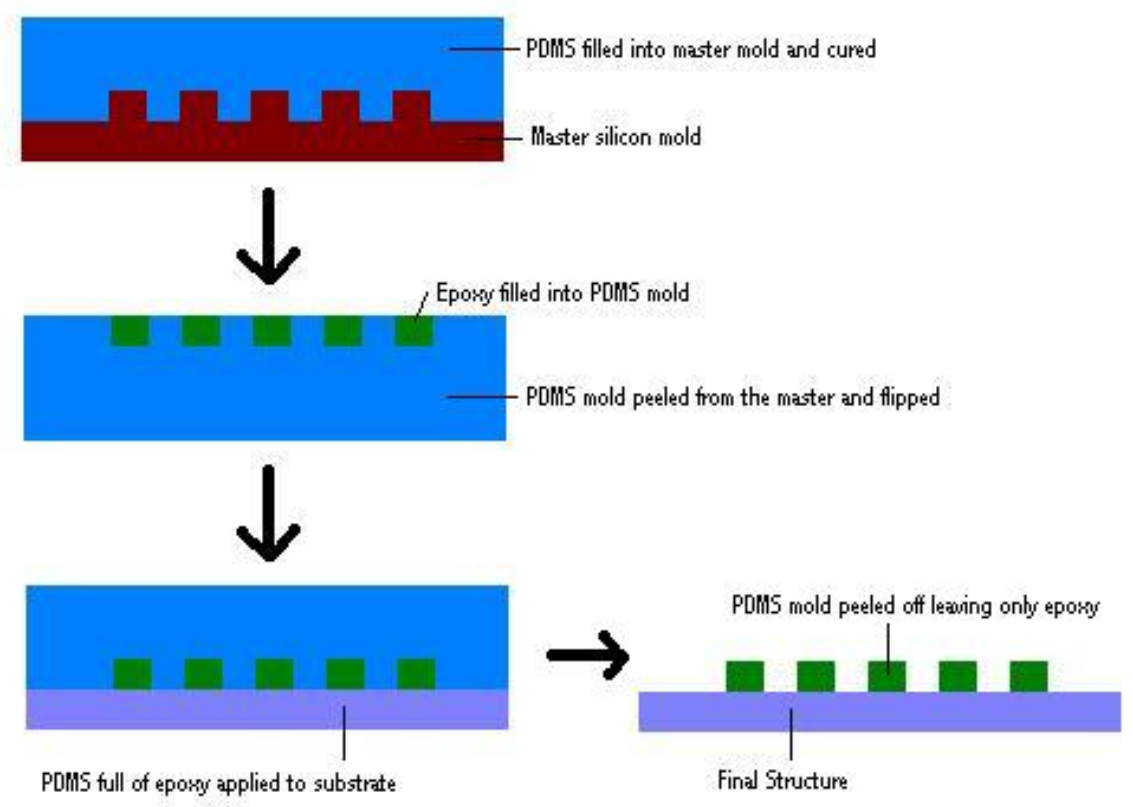

Figure 3.1 - Process used to build epoxy structures

The first stage of the process was creating a positive image of the final desired structure. The $0.4 \mu \mathrm{m}$ periodicity samples were made at Sandia National Laboratory with this use of electron beam lithography on a silicon wafer. The $1.0 \mathrm{~mm}$ and $2.5 \mathrm{~mm}$ periodicity samples were fabricated using standard photolithography techniques on a silicon wafer at the National Science Foundation laboratory at Iowa State University.

The next stage of the process was creating a negative of the final structure, using Dow Corning - Sylgard(R) 184 Silicone Elastomer Curing Ag (PDMS), which was poured into the 
patterned silicon wafer. The PDMS was made with a mixture of ten parts resin to one part hardener and was poured into a metal ring around the positive structure on the silicon wafer. The ring was held in place by the PDMS. The sample was left on a flat surface for an hour to allow for the escape of bubbles and then it was placed in a furnace at $75^{\circ} \mathrm{C}$ for five to six hours to set. When the PDMS was left in the furnace longer than a day or two, samples were more difficult to infiltrate because of different surface interaction with the epoxy - large amounts of the epoxy would stick in place on the PDMS, prohibiting the back and forth motion necessary to fill the grooves. After the PDMS rubber was set, it was cut out of the metal ring with a razor blade and labeled with the date and a letter corresponding to the silicon mold on which it was formed. The negative of the mold was placed in the furnace face up on a glass plate at $65^{\circ} \mathrm{C}$.

The next stage of the process involved infiltrating the PDMS negative mold with epoxy. Tra-Bond 2115 and Tra-Bond F113 epoxies manufactured by Tra-Con were used for the infiltration; the only difference between the two was the viscosity $-250 \mathrm{cps}$ for the 2115 and $180 \mathrm{cps}$ for the F113. The epoxy was mixed at a ratio of 2.7-2.8 parts resin to 1 part hardener. There were three methods used to create the $1^{\text {st }}$ layer of the structure. Two of these methods allowed for the application of the layer onto any type of substrate and the other built the layer on an epoxy substrate of the same material of the bars. The first method of creating a one-layer sample was pulling the PDMS from the furnace and placing an excess amount of epoxy on the center of the negative mold. The PDMS was then immediately placed face down on a glass slide, ensuring that the entire mold was in contact with the slide and that no bubbles appeared in the patterned area. After curing for two or three days, the PDMS mold was slowly pulled from the glass slide in the direction of the bars, leaving the epoxy behind on the glass. The slide was finally placed in a dessicator. Another method of making the first layer involved placing a washer over the sample area on the PDMS and then filling the washer with epoxy. When cured, the washer was pulled from the PDMS, leaving a $1^{\text {st }}$ layer of epoxy bars on an epoxy substrate inside the metal washer. These first two methods could only be used to create a one-layer sample. A more complicated procedure was necessary for subsequent layers and a first layer without an epoxy film underneath it. Using a small metal wire with a hardened ball of epoxy at the tip, the fresh epoxy was 
applied in a slow sweeping motion back and forth across the pattern perpendicular to the grooves. The number of times that the epoxy was swept over the pattern was varied in order to find an optimal procedure to fill the mold completely without overfilling. Variations of this procedure are discussed in the results section.

\begin{abstract}
Alignment
In order to align the epoxy bars to make two or more layer structures, a mechanism was designed to make use of the diffraction patterns exhibited by the structure when exposed to a laser beam. The design allowed for translational motion on three axes and rotational motion in a plane perpendicular to the normal of the patterns. In this manner, the two patterns could be lined up exactly to create the correct geometry for the final structure. The procedure for using the laser alignment is outlined below. Currently, the system is only capable of $2^{\text {nd }}$ layer alignment.

After the PDMS mold was filled with epoxy, it was set out for two to six hours to allow it to partially cure until it became very viscous. The edges of the mold were cut to make removal from the alignment device easier, and the mold was placed face down in the circular opening at the top of the aligner. A previously made one-layer structure was placed face up on a cone attached to the three-dimensional translation mechanism, and the two structures were brought close to contact by using four set screws on the side of the aligner. The two patterns were aligned on top of each other with the use of the side-to-side and front to back motion of the translation device. A $670 \mathrm{~nm}$ diode laser was then positioned above the two patterns and shone through them to create a diffraction pattern on a screen below. A grid on the screen was used to align the patterns 90 degrees from each other by rotating the PDMS mold until the first and second order diffraction dots were perpendicular to each other in each direction. When the samples were aligned, they were placed into contact using the third direction of motion of the translational device and removed through the top of the laser alignment system. The patterns were checked to ensure that full contact was achieved across the entire sample and they were set to cure for three days before peeling off the PDMS mold, leaving behind a two-layer structure of epoxy.
\end{abstract}


Alignment was also achieved in the NSF laboratory with the use of a mask alignment device. This equipment made it possible to view both the $1^{\text {st }}$ layer sample and the PDMS containing epoxy superimposed on one another before contact was made. The PDMS mold was placed on the vacuum assisted chuck upside-down, and the $1^{\text {st }}$ layer sample was placed on the bottom plate. A microscope was used to focus on both of the layers as they were moved closer towards contact with the use of a vertically translational mechanism. When the layers were close enough, they could both be focused at the same time and aligned before final contact.

\section{Slurry Infiltration}

The slurry infiltration procedure was performed by Henry Kang, who provided the following procedure information. A five to forty weight percent colloidal suspension of titania nanoparticles was made with DI water. The suspension was centrifuged at 3000 $25000 \mathrm{rpm}$ to remove large particles and agglomerates so the resulting suspension would contain only small particles $(<1 \mu \mathrm{m})$ that could infiltrate into the cavities of the epoxy mold. The particle weight percent of the centrifuged suspension was usually reduced significantly and could not be used to infiltrate the epoxy mold efficiently. Thus, the centrifuged suspension needed to be concentrated again by slowly evaporating the excess DI water in a temperature-controlled oven. The suitable particle weight percent for infiltration was determined to be around $0.05 \%$, whereas two drops of the suspension sufficiently filled one layer of the epoxy mold. The $\mathrm{pH}$ of the suspension was controlled to be between $7 \sim 11$ using ion exchange resins. Small amount of dispersants were added to help to prevent the reagglomeration, and binders were added to increase the mechanical strength of the green state.

A round metal washer with an area slightly bigger than the epoxy pattern was placed around the epoxy pattern with a magnet holding it on the other side. Two to three drops of the centrifuged and re-concentrated titania suspension were dropped into the washer, and the drops were dried at temperature around $25 \sim 35{ }^{\circ} \mathrm{C}$ for $30 \sim 60$ minutes. The above process was repeated for infiltrating multiple layers. After drying of the infiltrated epoxy mold, isostatic pressing was used to further densify the material. Finally, the sample was fired to 550 degrees Celsius with a ramp-up time of 8 hours and a soak time of 5 hours to remove the 
epoxy mold and to sinter the titania structure. The remaining titania bar structure was the inverse of the epoxy bar structure.

\section{Sol-gel Infiltration}

The following sol-gel procedure was also performed by Henry Kang. The sol (precursor) used for infiltration contained nanoparticles dispersed in a solvent (e.g. isopropanol, ethanol). Titanium diisopropoxide bis (2,4-pentadionate) (TDBP) and titanium (IV) isopropoxide (TIPP) were the two precursors primarily used in the procedure. TDBP has a relatively slow gelation rate and could be handled in air, while TIPP is extremely sensitive to moisture and needed to be handled in a dry nitrogen glove box. The precursors were introduced to the epoxy mold by spin coating. Before spin coating, the precursors were either diluted with appropriate alcohols (usually 2-proponol) or kept at the same concentration as received. Two to three drops of the solutions were dropped on the top of the epoxy pattern and the sample was immediately spun at a speed of 2000 to $4000 \mathrm{rpm}$ for one minute. Generally, the slower the spinning speed, the more the precursor material was filled in the epoxy mold. Thus, the spinning speed was set depending on the characteristics of the epoxy mold. For instance, one would want to set a high spinning speed to coat a one-layer mold and set a lower spinning speed to coat a multiple-layer mold. After spin coating, the sample was dried in air for a couple hours or until the gelation reaction was completed. The drying was also attempted at an elevated temperature of $100^{\circ} \mathrm{C} \sim 110^{\circ} \mathrm{C}$. After drying, the sample was fired to $550^{\circ} \mathrm{C}$ with a ramp-up time of 8 hours and a soak time of 5 hours to remove the epoxy mold and to convert the precursor into titania. The remaining titania bar structure was the inverse of the epoxy bar structure.

\section{Characterization Methods}

Several methods were used to determine the quality of the PBG samples. The simplest method was examining the samples under an optical microscope. The desired resolution dictated the equipment that was needed. Quick checks on the samples were 
performed with an Olympus 071 optical microscope, and more in depth analysis was done on either a Jeol JSM - 6100 Scanning Electron Microscope (SEM) or an R J Lee Instruments Personal Scanning Electron Microscope (PSEM). The use of these high-resolution machines however, required the samples to be coated with a conductive layer, which rendered them useless for further application.

The quality of the layered bar structure was also analyzed in a physics laser laboratory with the use of reflective diffraction detection. A $457 \mathrm{~nm}$ wavelength laser was aimed through a small pinhole to create a small spot size on the sample. The reflected diffraction pattern was collected in a hemisphere screen and recorded by a CCD camera. The experimental setup is shown in figure 3.2. This method determined the defect level of the sample by analyzing the intensity of radiation in between the principle diffraction dots.

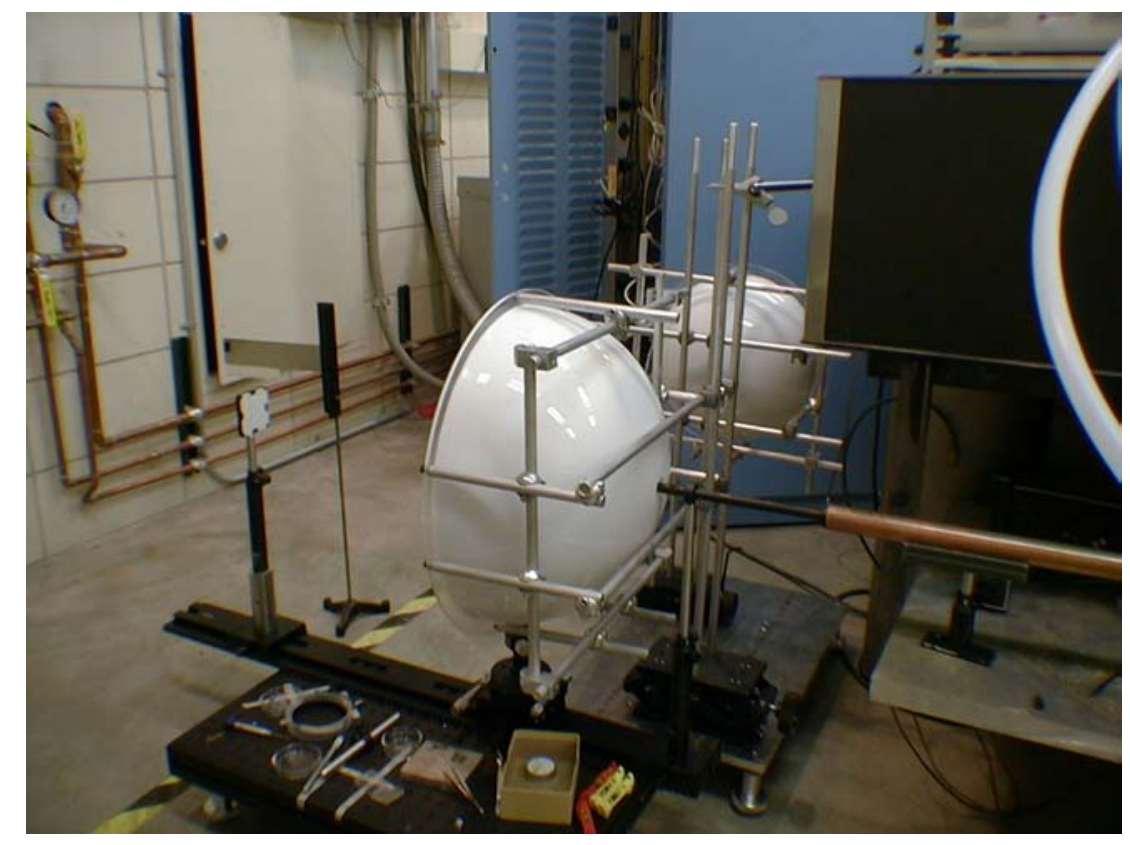

Figure 3.2 - Experimental setup used to make reflected diffraction pattern measurements 


\section{CHAPTER 4: RESULTS AND ACCOMPLISHMENTS}

\section{Experimental Equipment}

Research to date utilizing the proposed procedure has resulted in the fabrication of consistently good two-layer PBG samples with a periodicity of $2.5 \mu \mathrm{m}$ and $1.0 \mu \mathrm{m}$, and the preliminary experimentation with the fabrication procedure of the $0.4 \mu \mathrm{m}$ sample. The first step towards the production process of these samples was designing and building a clean box in order to isolate the samples from the dust and other airborne contamination in the laboratory. To do this, $3 / 8$ inch plexi-glass was used to make a 6 ' long by $2^{1} /{ }^{\prime}$ ' wide by 3 ' high box with fan towers in the top to blow air through dust filters. The air from the fans was meant to provide a positive flow of clean air through the box so that when the door in the front is open for working purposes, only clean air was allowed into the work area. A picture of the clean box is shown below as figure 4.1 .

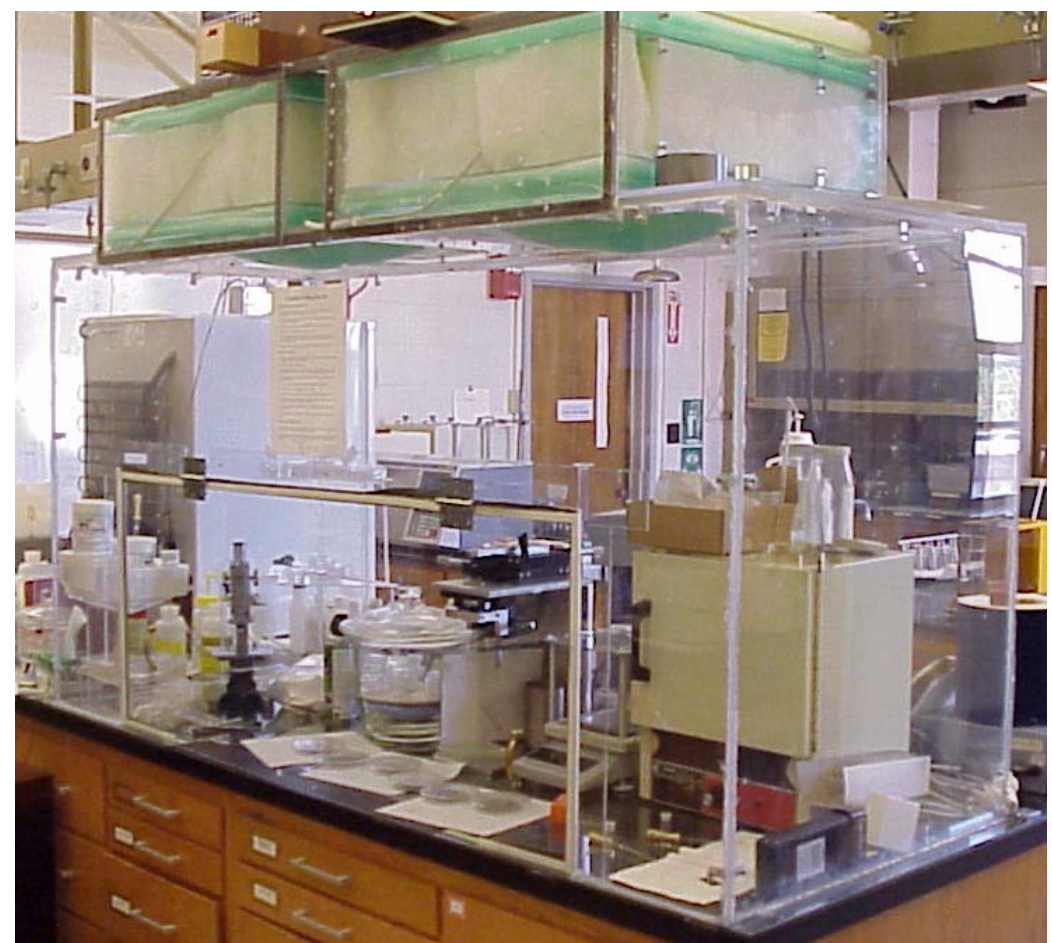

Figure 4.1 - Clean box built to house the fabrication process of epoxy molds 
It was also necessary to develop a method to align the layered structures 90 degrees from one another. The alignment system discussed previously was designed, which takes advantage of the diffraction properties of the layered samples. The alignment mechanism was designed to operate with rotational and translational microscope stages and machined by the metals development shop. A picture of the system is shown below as figure 4.2. The laser alignment system could be used to align the 2.5 and $1.0 \mu \mathrm{m}$ samples, but the diffraction dots were not visible through the $0.4 \mu \mathrm{m}$ samples because of the reduced bar spacing to the point that the $1^{\text {st }}$ order dots were diffracted at an angle greater than 90 degrees. It would be possible, however, to use a higher frequency laser for the 0.4 micron samples in order to be able to see the diffraction pattern. Alignment was generally successful for two-layer samples, but many there were many problems with aligning the first and third layers offset half of period from each other.

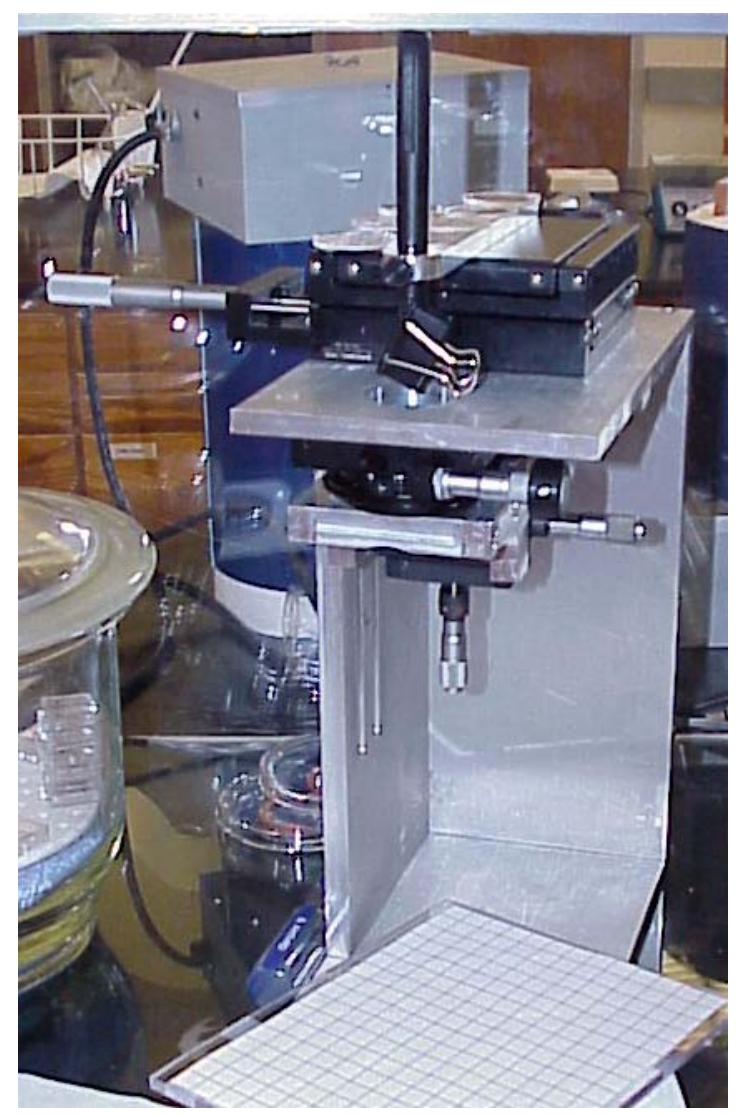

Figure 4.2 - Laser alignment system designed for the 2.5 and $1.0 \mu \mathrm{m}$ samples 


\section{Epoxy Infiltration}

\section{One Layer Methods}

Two procedures were mentioned earlier that dealt only with creating a $1^{\text {st }}$ layer sample: applying excess epoxy to the PDMS mold and then pressing it onto a substrate and filling a washer with epoxy on top of the PDMS mold. Both of these procedures resulted in a perfect, consistent $1^{\text {st }}$ layer. The problem with these methods was that neither of them resulted in an epoxy structure built directly upon a given substrate that would hold an infiltrated sample. For example, the one drop method would leave a small film between the bars and the substrate and the washer method resulted in a free standing structure with only epoxy for a substrate. The epoxy substrate was thought to be a good way to make several layer samples by infiltrating a two-layer structure on a glass substrate and a two-layer structure on the epoxy substrate to finally place both of them into contact and fire to obtain a four-layer titania network. This method however, was unsuccessful, and it was thought that large mass of the epoxy substrate was too much material to be evacuated from around the titania bars. The burning out of the epoxy seemed to destroy the top layers of the sample, leaving only remnants of the titania on the final substrate. After several experiments, it was found that the thin layer of epoxy left after the one-drop method really did not have any effect on the final fired sample. Therefore, it was considered the preferred method in fabricating one-layer samples.

\section{Multi-layer Methods}

The fabrication of samples comprising more than one layer proved to be the most difficult part of this project and became the main focus of this paper. The main problem in the mold production was that it was almost impossible to reproduce a previous procedure or even to achieve consistent results for samples being made at the same time. Several of the variables involved in the process are summarized in table 4.1. The table also lists an estimated rating of the importance of each variable and the ability to control that variable. 
The table is arranged by the strength of the effect that each variable contributes to the final mold, which is ranked on a scale from one to ten, one having very little effect and ten having a large influence on the quality of the mold. The "Ability to Control" column is also rated on a scale from one to ten, one being extremely difficult to control and ten being very easy to control. It is apparent from the table that the more important variables are the ones that are the most difficult to control, coupled with the fact that it was only possible to produce two to four molds a day, made it very difficult to set up any kind of design of experiments (DOE). Each variable is discussed here in more detail based on laboratory experience.

Table 4.1 - Variables involved in the mold production procedure

\begin{tabular}{|c|c|c|}
\hline Variable & Effect on Final Mold & Ability to Control \\
\hline & $\mathbf{1}$ (little) - 10 (large) & $\mathbf{1}$ (difficult) - 10 (easy) \\
\hline Viscosity of Epoxy (Two Choices) & 1 & 10 \\
\hline PDMS Furnace Time & 4 & 10 \\
\hline Particle Contamination & 4 & 8 \\
\hline PDMS Resin to Hardener Ratio & 4 & 10 \\
\hline Time Between Furnace Holds & 5 & 9 \\
\hline Time Between Application of Newly Produced Layer & 6 & 10 \\
\hline Sweep Speed & 7 & 6 \\
\hline Number of Sweeps Between Furnace Holds & 7 & 10 \\
\hline Furnace Temperature & 7 & 9 \\
\hline Ambient Temperature & 8 & 2 \\
\hline Humidity & 8 & 2 \\
\hline Number of Total Sweeps & 9 & 10 \\
\hline Epoxy resin to hardener ratio & 9 & 6 \\
\hline Temperature Change of Sample During Epoxy Application & 10 & 4 \\
\hline
\end{tabular}

The PDMS was easy to control and didn't have much effect on the epoxy structure. The resin and hardener ratio was easy to regulate because the amounts were large enough to accurately measure, and the furnace time frame needed to set the PDMS could range anywhere from four hours to two days. Before four hours, the PDMS would not be completely set and after two days of being in the furnace, it was more difficult to infiltrate the PDMS with epoxy. When infiltrating an affected sample, the epoxy would stick in place, becoming much less fluid than usual. In this state, it was impossible to remove the epoxy from the pattern, which ruined the entire sample. A possible explanation is that the length of furnace time affected the surface energy of the PDMS material because of bond changes. 
The extended high temperatures could have also caused alterations in the rubber material or structure, leading to porosity or defects that would attract and hold epoxy in place. Temperature was also a factor during the infiltration of PDMS with epoxy - liquids were observed to wet the PDMS more as temperature increased. This property was useful in increasing the capillary action needed to fill the grooves in the PDMS with epoxy.

The resin to hardener ratio for the epoxy was extremely difficult to regulate because of the small amounts involved. Only about $30 \mathrm{mg}$ of epoxy was needed for the procedure, which was difficult to measure on the available scale in the lab. Factors such as air pressure from the tower fans and general vibrations in the lab caused noise in the reading from the scale around $+/-8 \mathrm{mg}$, which made accurate mixtures difficult to make. Generally, it was possible to tell whether or not the epoxy mixture was resin dominated or hardener dominated. The effects of a poor mixture included increased curing time and odd overfilling tendencies during cooling, which could be responsible for the variance observed in final molds with respect to the more controllable variables.

The ambient temperature and humidity of the laboratory were the most difficult parameters to control in the process. The Gilman facility used was not well suited for climate control, and therefore the conditions in the laboratory continually varied. Samples tended to be of higher quality in low humidity and ambient temperatures around $65^{\circ} \mathrm{F}$. High humidity also caused problems for the adhesion of the structure to the glass substrate. The sample would lift off of the glass as subsequent layers were added if subjected to high humidity. To solve this problem a sample was either kept in a dessicator or was left adhered to the PDMS until it was necessary to remove.

Most of the critical parts of the procedure involved the temperatures, times, and number of sweeps across the sample during the application process. The amount of epoxy that remained within the grooves was strongly dependent on procedure. The final objective in making the layer was finding the optimal amount of infiltration so that the grooves were completely filled; yet not overfilled. This was a delicate balance as it was necessary to find the correct combination of all of the variables that worked together to output the exact amount of epoxy necessary to just fill the mold. From experience, it seemed that the most important variable to consider was the temperature of the PDMS during and just after the 
infiltration step. Temperature affected many aspects of the experiment. First of all, the viscosity of the epoxy decreased with increasing temperature, and the cure time drastically decreased with increased temperature. Also, the ability of the epoxy to wet the PDMS seemed to increase with the increasing temperature. For these reasons, a furnace temperature between $60-70{ }^{\circ} \mathrm{C}$ was used to heat the samples between infiltrations. While the epoxy was in the furnace, it would partially cure, and the PDMS would also be heated so that the new epoxy would be extremely fluid while infiltrating the next round. Several iterations of this process were necessary, which is because the epoxy would shrink considerably while curing. The ability to keep the PDMS hot throughout the infiltration was varied by setting it on a larger/smaller plate that would retain more/less thermal energy during the steps of the procedure outside of the furnace. It was suggested that a hot plate or some sort of heating device could be used to maintain a higher mold temperature. However, if the sweeping of the epoxy across the mold were finished while everything was hot, the epoxy did not stay in the grooves. It is thought that it is necessary that the epoxy cool during application to promote adhesion because of the higher viscosity of the epoxy at a lower temperature. For this reason, epoxy was applied PDMS on the hot glass until the final pass across the sample area, at which point the PDMS was transferred to a cold glass plate to cool it rapidly.

The parameters that could be controlled the best and also had a large effect on the final structure involved the sweeping of the epoxy over the pattern. The rates both side-toside and forward and back affected the ability of the epoxy to fill the grooves. The number of sweeps across the pattern each step and the number of steps affected the total amount of epoxy that finally remained in the mold. As stated earlier however, the temperature of the epoxy was a large consideration that could totally change the necessary total number of sweeps to fill the mold. Figures 4.3 - 4.8 show PSEM images of several 2.5 micron samples and their respective sweeping procedures. Figures $4.9-4.13$ show several 1.0 micron samples and their respective sweeping procedures. Figures $4.14-4.14$ show a few 0.4 micron samples and their respective procedures. Most of the samples were two-layers because of the problem with alignment, but one four layer sample is also shown. A sweep is defined as a pass back and forth across the sample area. 


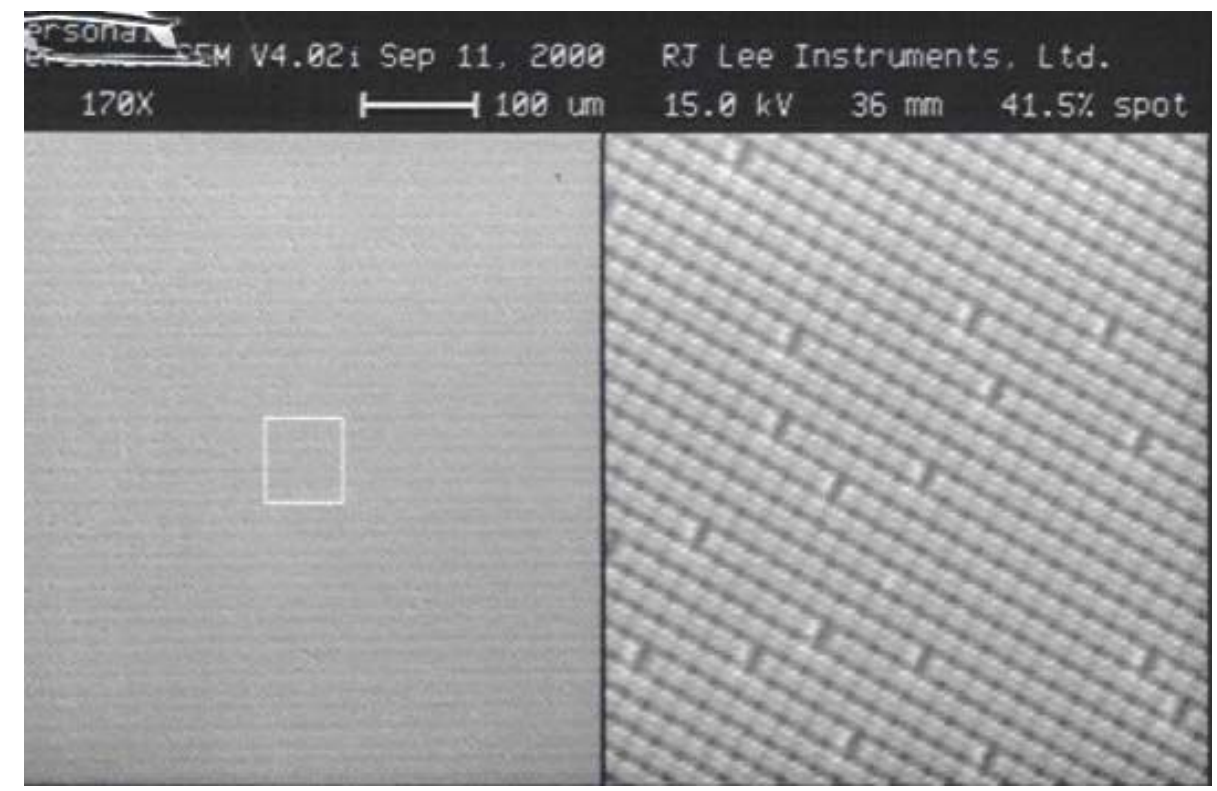

Figure 4.3 - Two-layer 2.5 micron epoxy sample made on 9-6-2000. Epoxy was applied with sweeps of $4.5,3.5,2.5$, and 1.5 times with approximately 5-10 minutes in the furnace in between applications. The sample was left in ambient air for 3.5 hours then 3.5, 1.5 sweeps were applied.

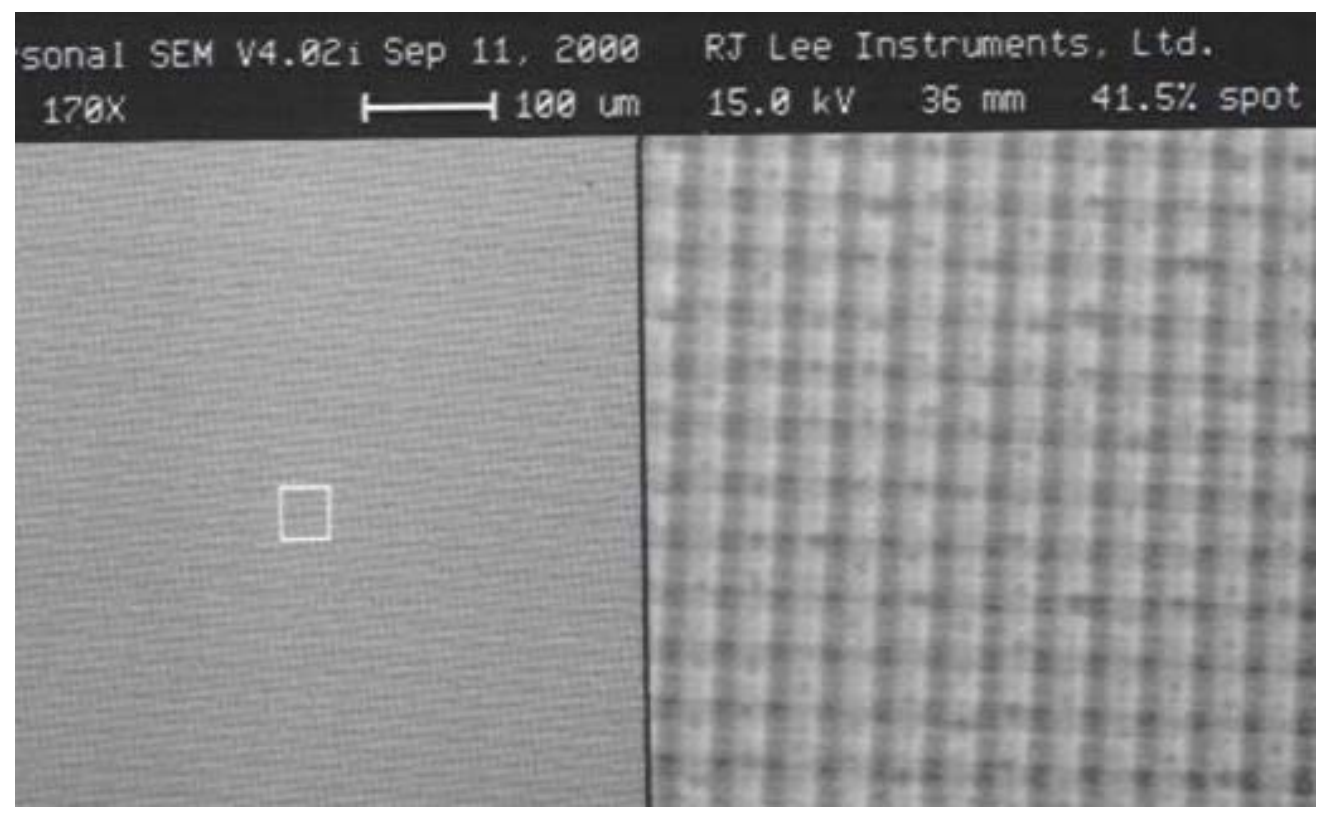

Figure 4.4 - Two-layer 2.5 micron epoxy sample made on 9-7-2000. Epoxy was applied with sweeps of 7.5 and 2.5 times with 5-10 minutes in the furnace between applications 


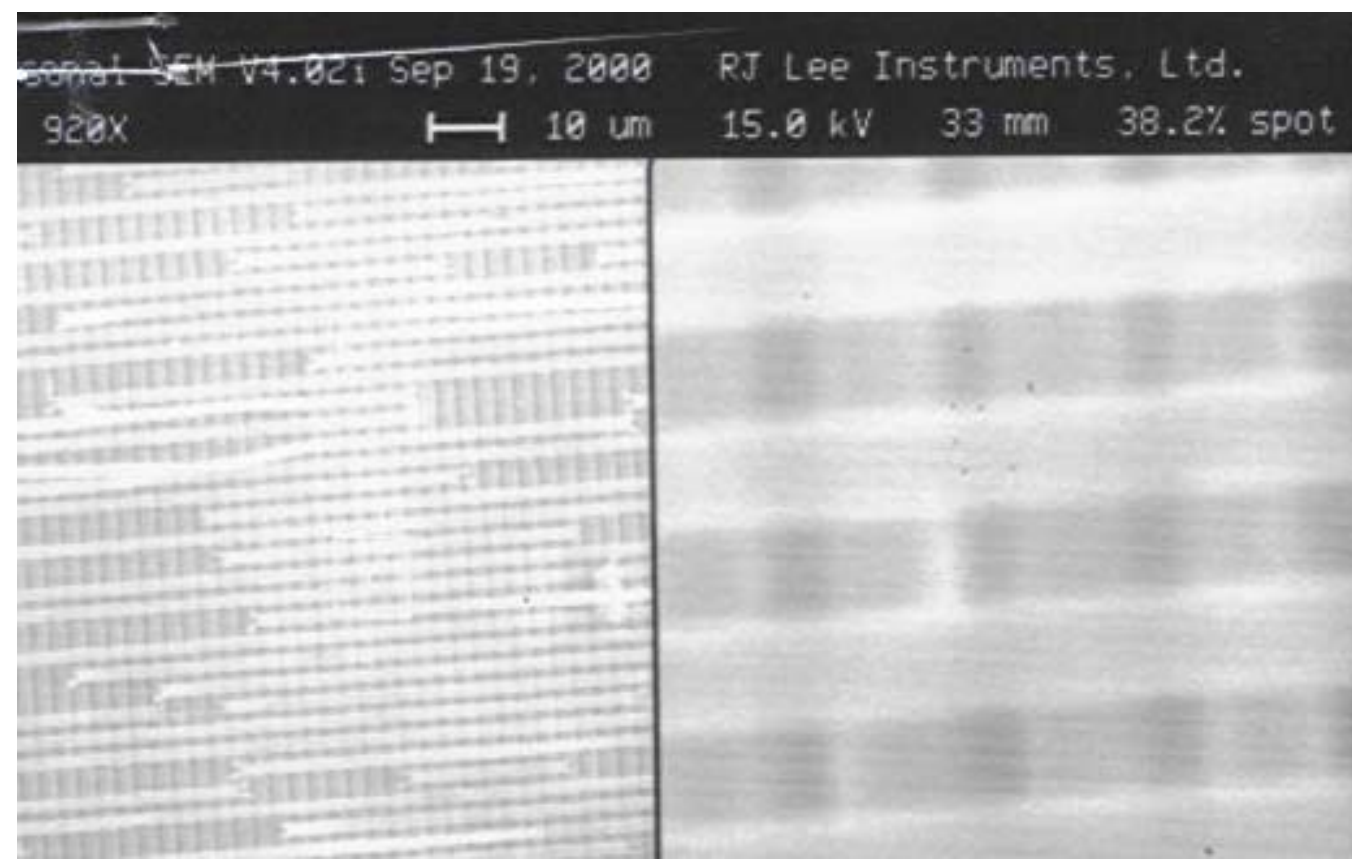

Figure 4.5 - Two-layer 2.5 micron epoxy sample made on 9-15-2000. Epoxy was applied with sweeps of 6,6 , and 6 times with 5-10 minutes in the furnace between applications.

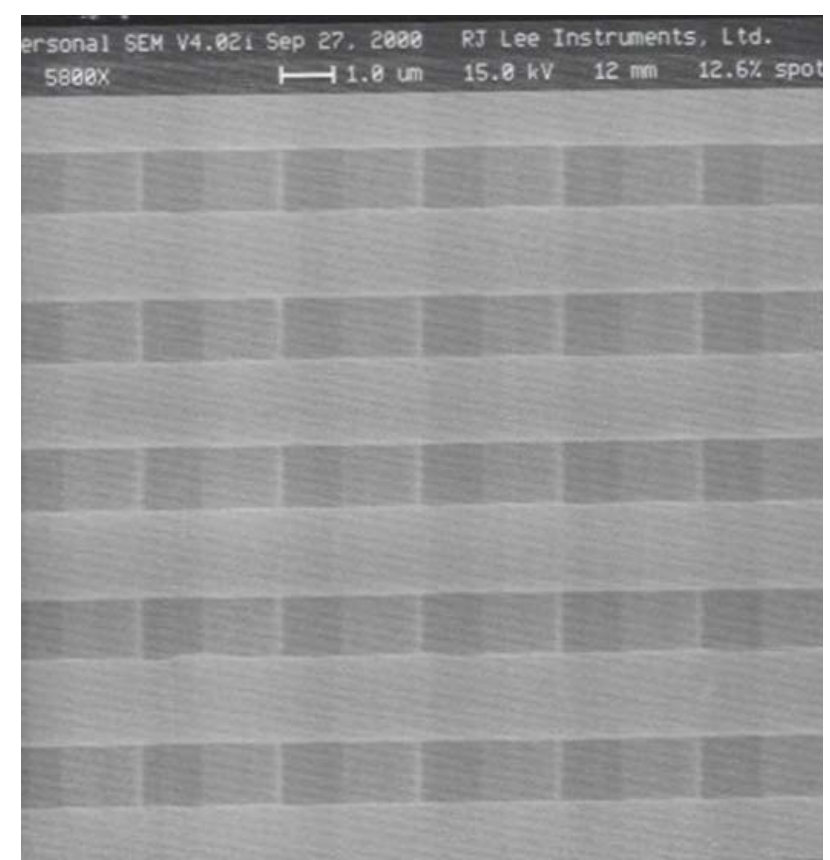

Figure 4.6 - Two-layer 2.5 micron epoxy sample made on 9-22-2000. Epoxy was applied with sweeps of 4, 3, 2, 1 times. New epoxy was mixed and applied again with sweeps of 4, 3, 2, 1 times. The sample was in the furnace 5-10 minutes between applications and slightly longer during the mixing of the second epoxy. 


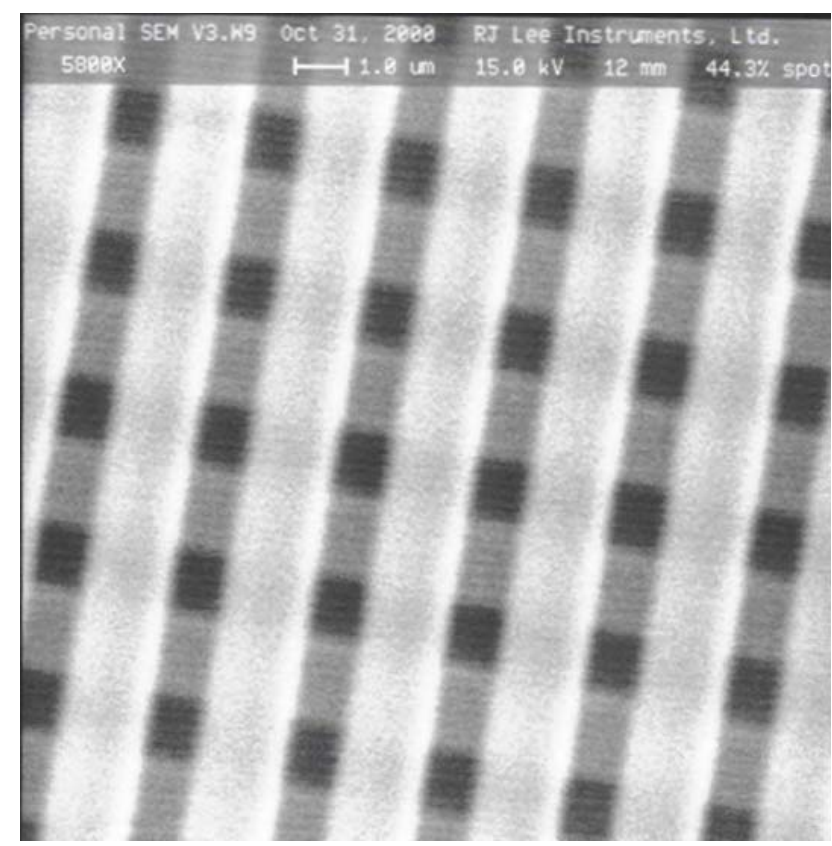

Figure 4.7 - Two-layer 2.5 micron epoxy sample made on 10-18-2000. Epoxy applied 4.5, 3.5, 2.5, and 1.5 times, was mixed again and applied 4.5, 3.5, 2.5, and 1.5 times again. Furnace times of 5-10 minutes.

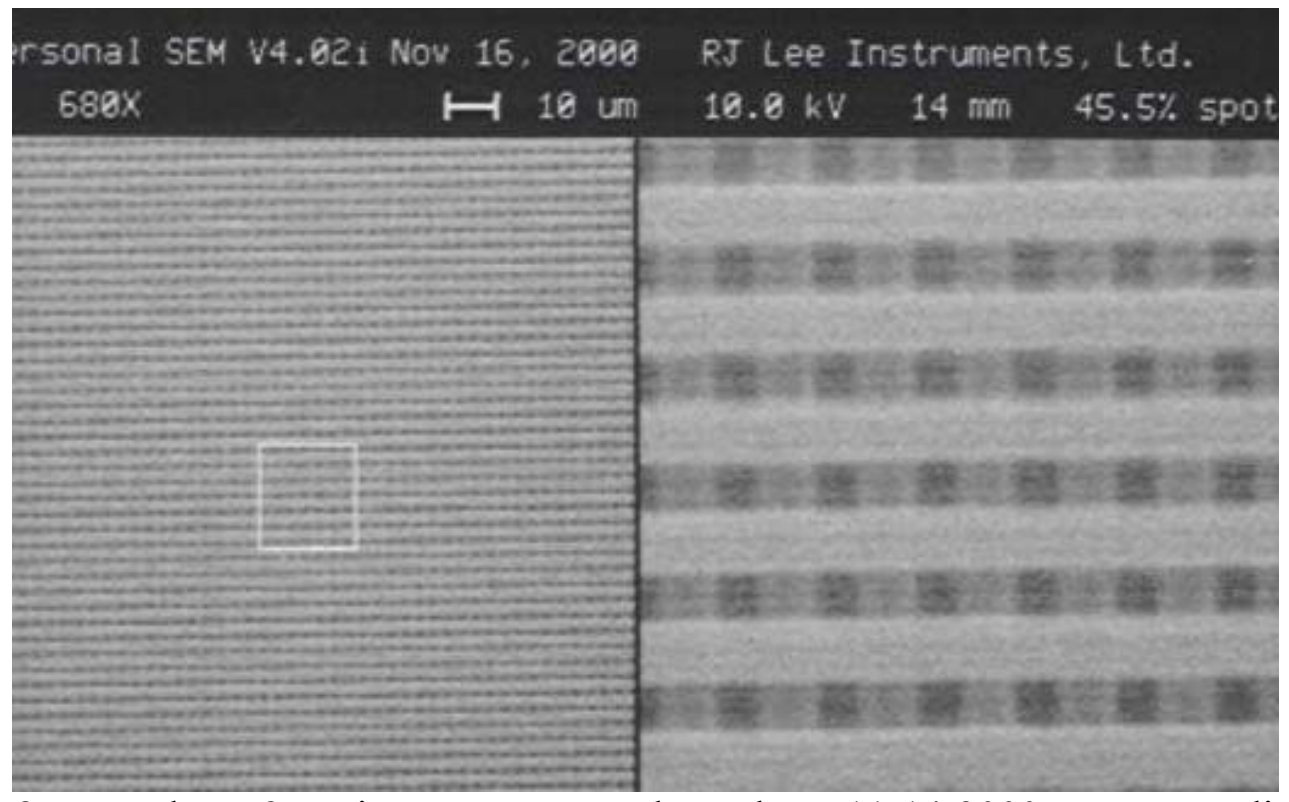

Figure 4.8 - Two-layer 2.5 micron epoxy sample made on 11-14-2000. Epoxy applied 4.5, 3.5, and 2.5 times, was mixed and applied again 3.5 and 2.5 times slowly. Furnace times 510 minutes between applications. 


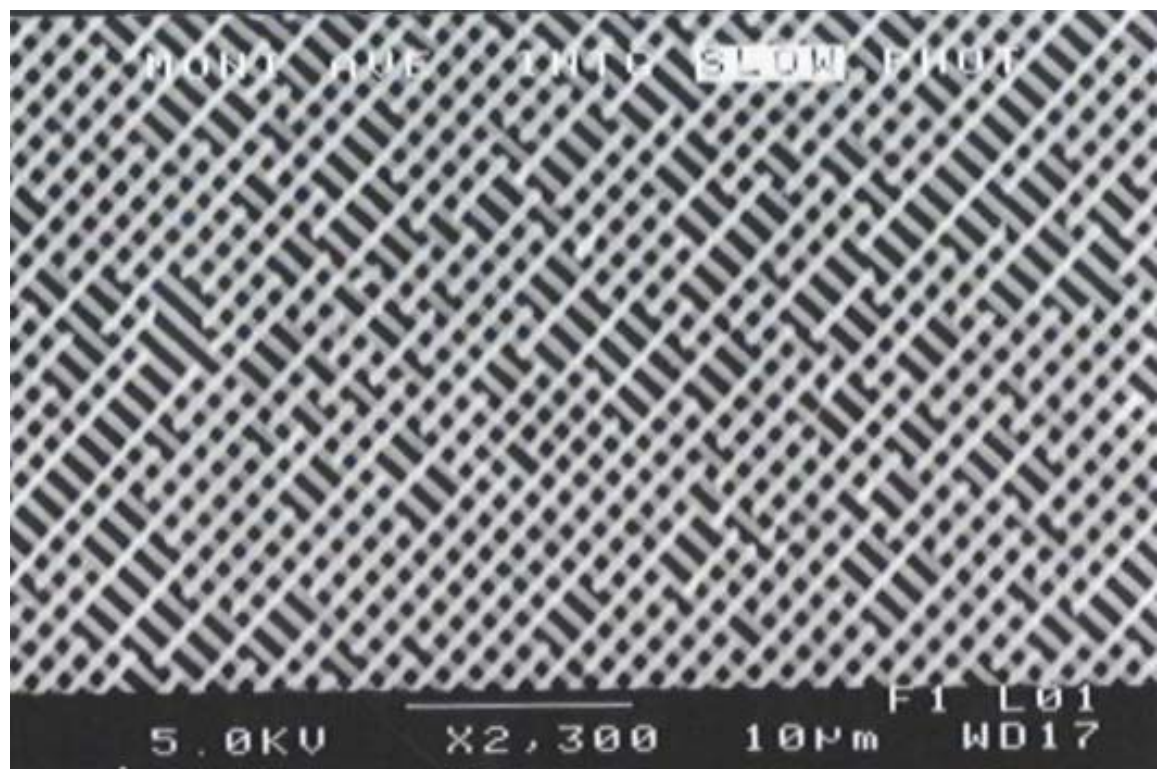

Figure 4.9 - Two-layer 1.0 micron epoxy sample made on 1-30-2001. Epoxy applied 4, 4, 2 times with 5 minutes in the furnace between each application.

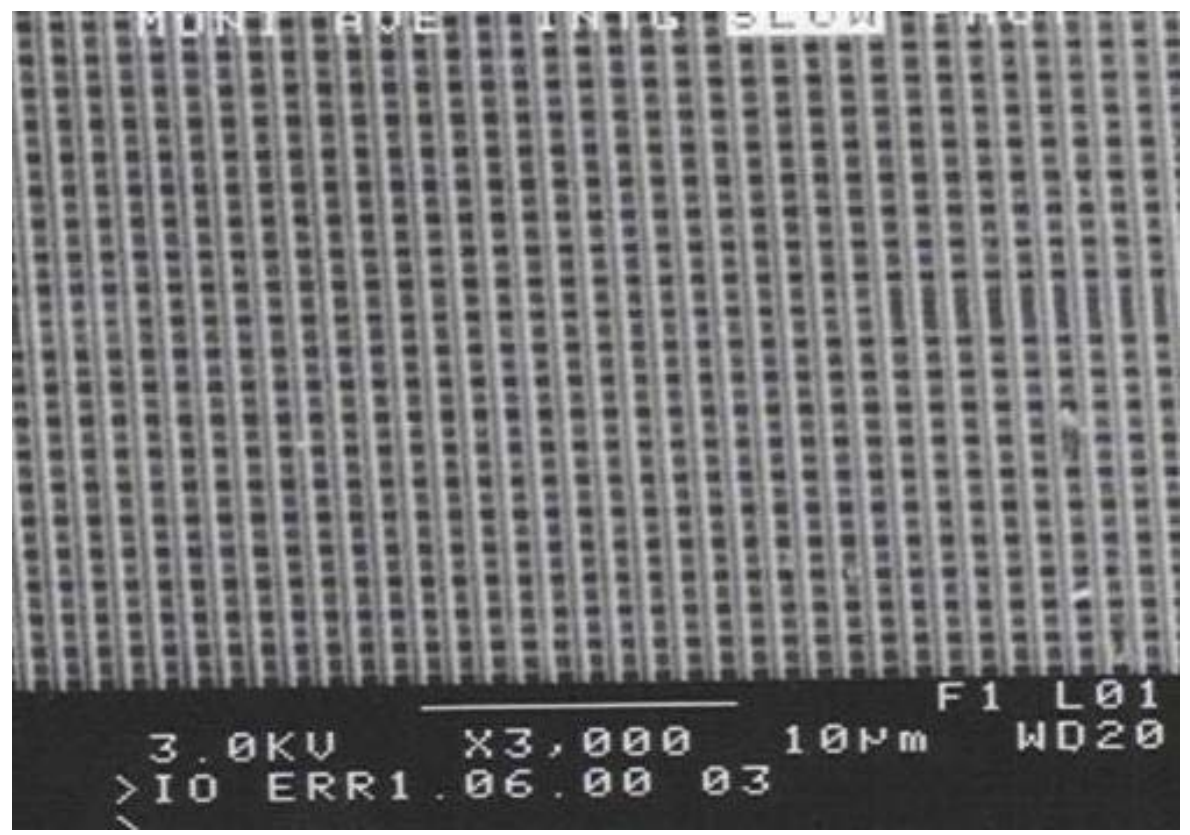

Figure 4.10 - Two-layer 1.0 micron epoxy sample made on 2-6-2001 using a lower viscosity epoxy. It was applied 4 and 4 times, then new epoxy was mixed and applied 4 and 3 times then mixed again and applied 3 times across. Furnace times were around 5 minutes. 


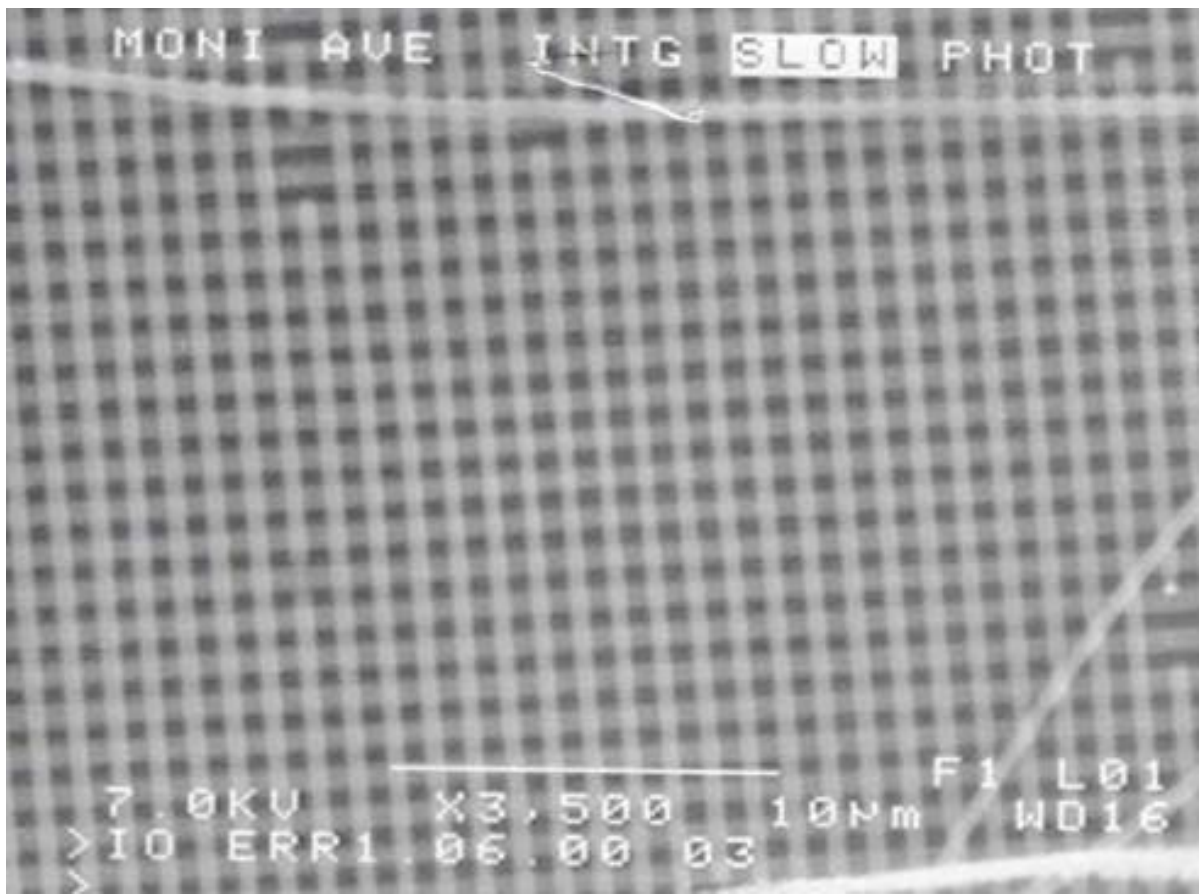

Figure 4.11 - Two-layer 1.0 micron epoxy sample made on 2-11-2001. Epoxy applied 4, 4, and 3 times then 4 and 4 times after mixing new epoxy.

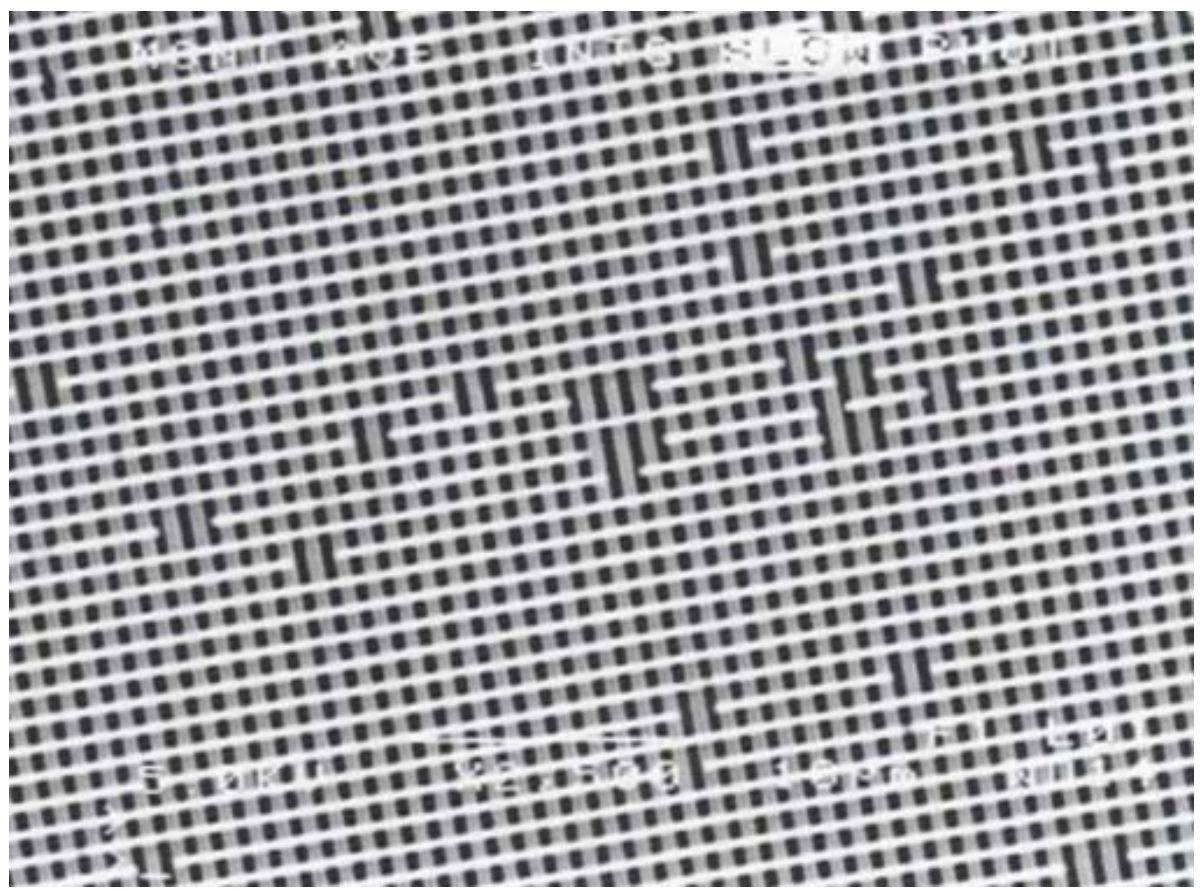

Figure 4.12 - Two-layer 1.0 micron epoxy sample made on 2-12-2001 with higher viscosity

epoxy. Epoxy applied 4, 4, and 4 times then 4 and 4 times after mixing a new batch.

Furnace times of 5 minutes between applications. 


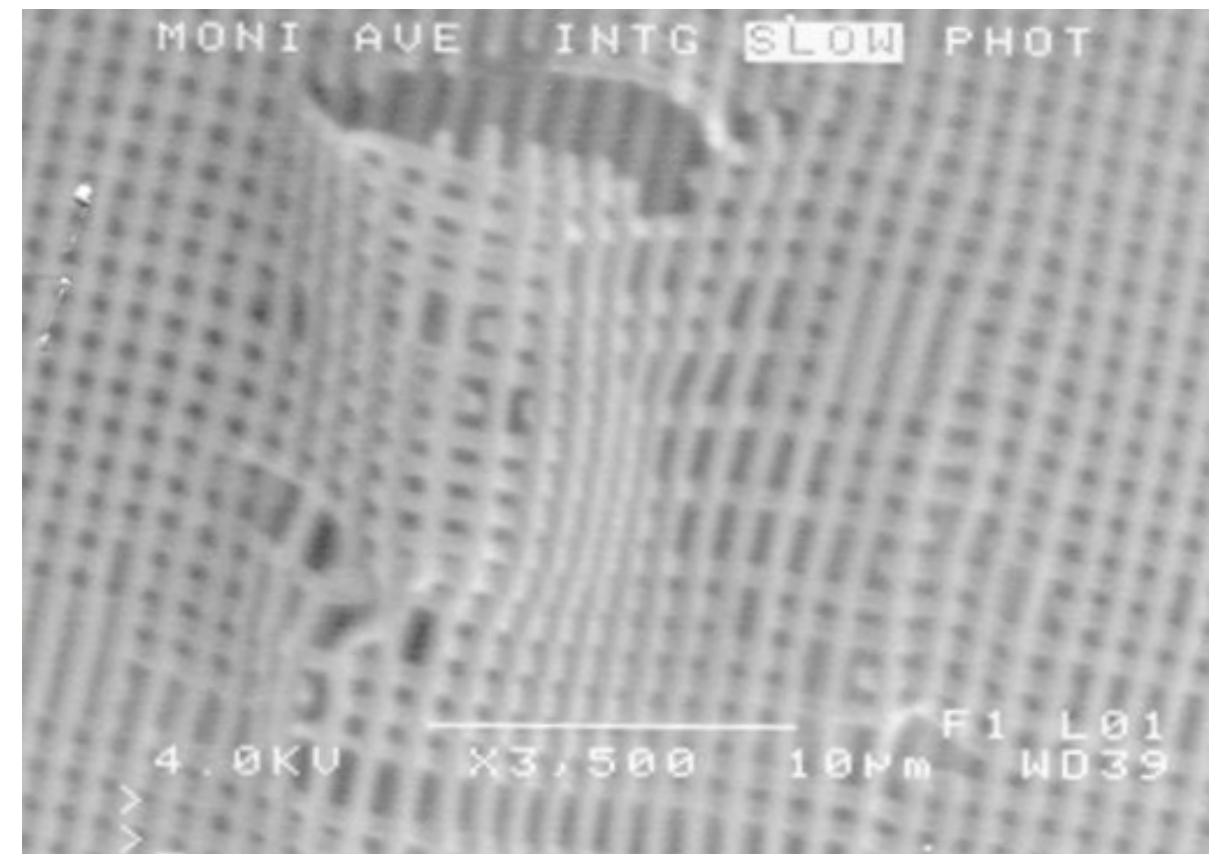

Figure 4.13 - Four-layer 1.0 micron epoxy sample (defect region shown for visibility of lower layers) $4^{\text {th }}$ layer made by applications of 4 and 3 then 4 and 3 after a second mix.

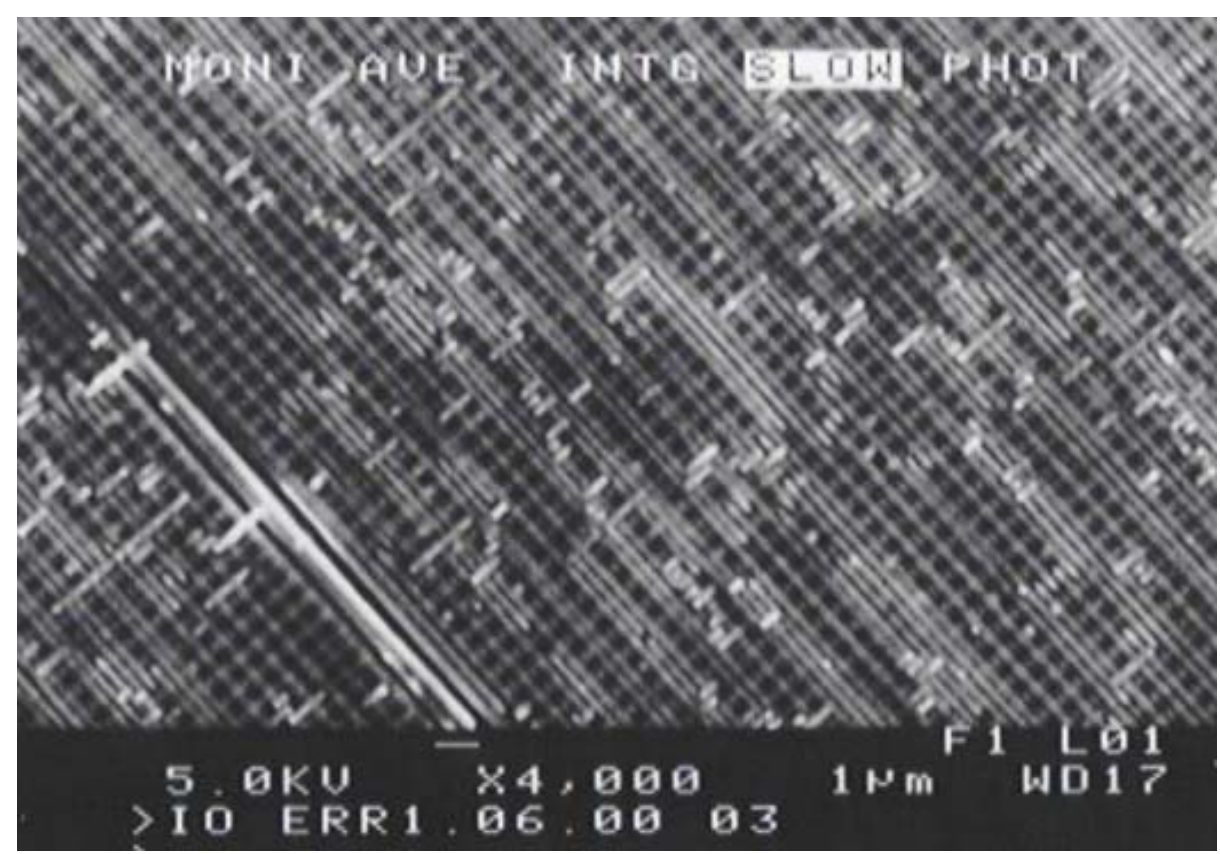

Figure 4.14 - Attempted two-layer 0.4 micron epoxy sample made on 1-30-2001. Epoxy applied 3.5, 3.5, and 2.5 times across the sample. Furnace times of 5 minutes in between applications. 


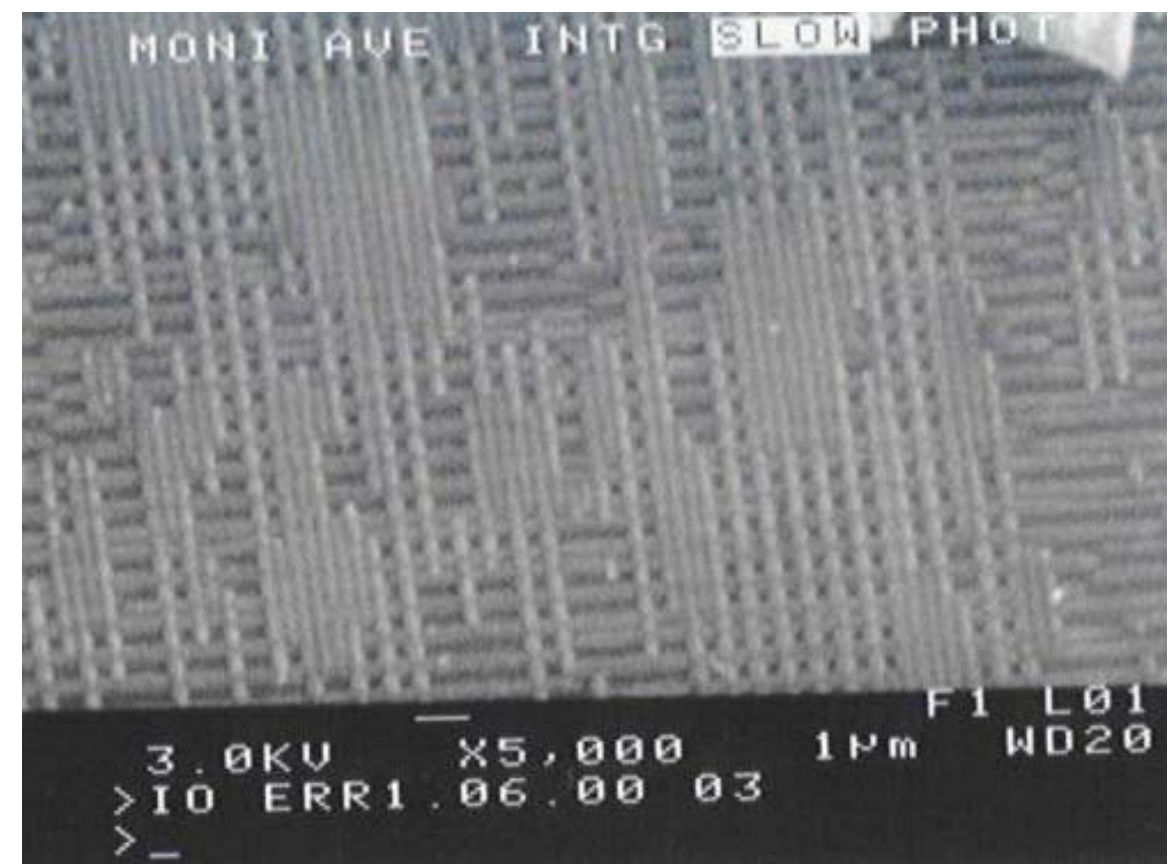

Figure 4.15 - Attempted two-layer 0.4 micron epoxy sample made on 2-6-2001. Epoxy applied 3.5 and 2.5 then mixed then 3.5 and 2.5 then mixed then 3.5 times across. Furnace times of 5 minutes in between applications.

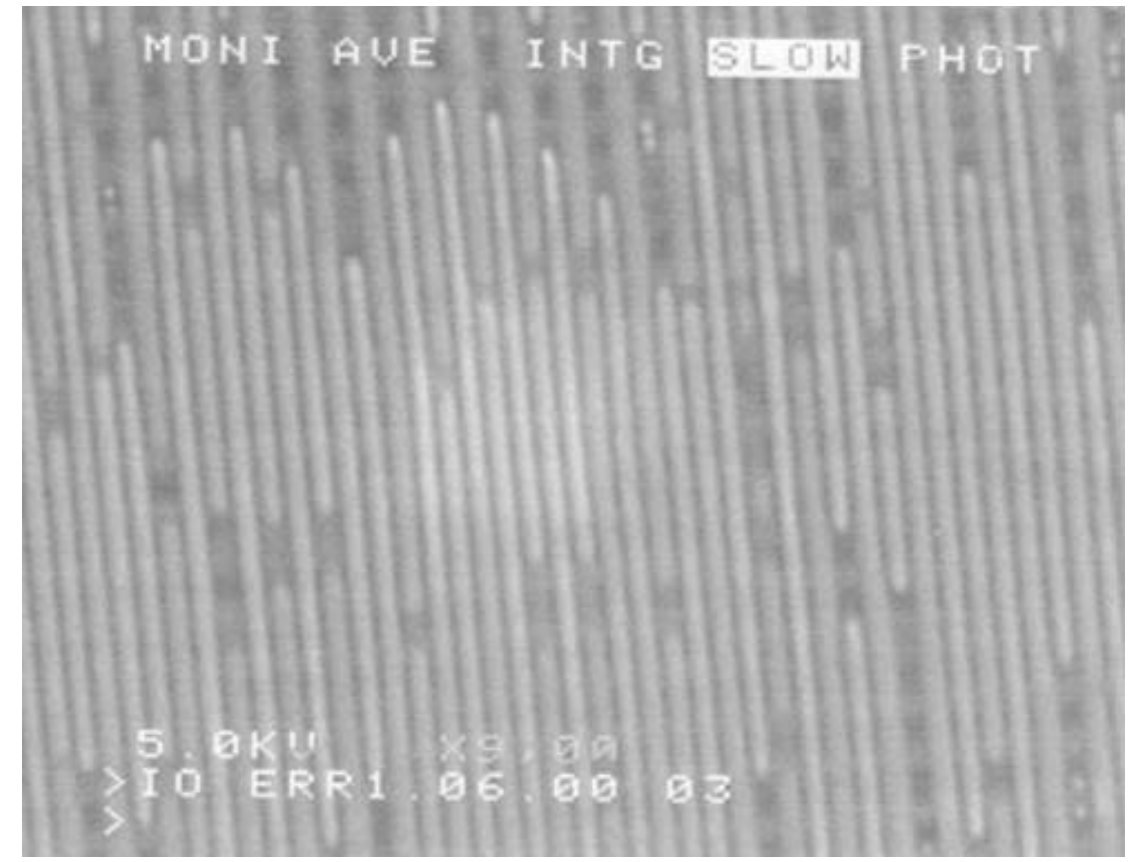

Figure 4.16 - Two-layer 0.4 micron epoxy sample made on 2-12-2001 with the higher viscosity epoxy. Epoxy applied 4, 4, 4, 4, and 4 times before another epoxy mixture was made and then 4, 4, and 4 times more. Furnace times of 5 minutes again. 
The procedure for making samples was generally modified each time, depending on previous outcomes and the current progress as monitored with a 10x optical microscope inside the clean box. Since there were so many uncontrolled variables involved, it was difficult to state a specific ideal experimental procedure. For the 2.5 micron samples, the most successful process used two mixes of epoxy. The first mixture was used to sweep back and forth across the pattern $4,3,2$, and 1 time with 5 minutes of furnace time in between the infiltrations. At this point, the epoxy was generally too viscous to be useful because of curing effects, and a second batch was mixed. The second epoxy mixture was used for about 4 , then 3 sweeps across the pattern. For the 1.0 micron samples, the most successful process only required one epoxy mixture. It was used to sweep across the sample 4, 4, then 3 times across the pattern with 5 minute furnace holds in between the infiltrations. It was still necessary to monitor the progress of the epoxy-filling ratio at every step of the procedure. One could determine how well the grooves were filling and modify the process as necessary. Usually, after the infiltrated sample had been cooled for 5-10 minutes, the epoxy would ball up as if the sample is overfilled. This effect would diminish and disappear as the epoxy cured.

Overall, the 1.0 micron samples were much easier to infiltrate than the 2.5 micron structures. This was likely to be caused by the increased capillary action in the smaller channels and also the smaller amount of epoxy necessary to fill the molds, which lessened the effect of expanding and contracting that took place during heating, cooling and curing. Also, fewer sweeps were necessary to fill the 1.0 micron sample The 0.4 samples were not at all successful, which is most likely attributed to a defective master silicon mold. The 0.4 samples have seemed to get progressively worse each time as well, which suggests that the use of the master mold causes contamination that affects subsequent molds. The PDMS molds were viewed during the infiltration process and they appeared to be overfilled and underfilled at the same time, meaning the epoxy would ball up on the sample. This was common for the other samples only if the grooves were already completely full, but the 0.4 micron mold would exhibit this non-wetting property even though the grooves contained no epoxy. 


\begin{abstract}
Alignment
Two alignment procedures were explained earlier in this paper: laser alignment and optical alignment with the use of a mask aligner. It was noted that the laser alignment system was capable of only second layer alignment, but third and subsequent layer alignment was possible if half period spacing between even and odd layers was not a consideration. The optical system was able to accommodate the half period spacing requirement but not completely. There was a problem with the matching between layers already applied to the substrate and the matching grooves in the PDMS mold. Because the PDMS was flexible, it did not exhibit the same periodicity or bar spacing all the time, which could have been caused by the deformation of the mold during alignment. For example, a pattern that was aligned perfectly on one edge with the mask aligner would be out of alignment as it was shifted towards the middle. Therefore, there were regions of good alignment and regions of bad alignment. Thicker PDMS molds were used in an attempt to alleviate this effect, but it could not be completely eliminated.
\end{abstract}

\title{
Slurry Infiltration
}

Henry Kang, who is the source for the following information, did most of the work on the slurry and sol gel infiltration of the samples. Several modifications have been considered in the attempt to produce a titania slurry that would be best suitable for infiltrating the epoxy bar structure. These included varying the $\mathrm{pH}$, adding dispersants or adding binders into the slurry. So far these modifications have not seemed to help produce a better titinia bar structure at the end. The most successful two-layer titania structure produced used a plain centrifuged suspension without any additives, and the sample was isostatic pressed prior to the firing. The SEM images of the structure are shown below in figure 4.17. Infiltrating a four-layer epoxy mold was also attempted and the resulting titania structure is shown in Figure 4.18. Notice the titania bars in the four-layer structure appear to be wider (indicating excess amount of material), have rough edges, and are slightly folding inward. Also, the bottom layers seem to have less material than the top layers. Although it is not clear what 
caused the imperfect structure, capillary actions between the mold and the slurry droplets, and air trapped within the molds could all be the potential factors that prevent the slurry from infiltrating downward. This resulted in less material in the bottom layers and excess amount of material on the top layers. In the drying step, re-agglomeration could have occurred and induced the formation of particles with dimensions larger than the feature size of the epoxy mold. The large particles would be trapped at the top without being able infiltrate to the bottom layers of epoxy, which could have resulted in an excess amount of material at the top layer.
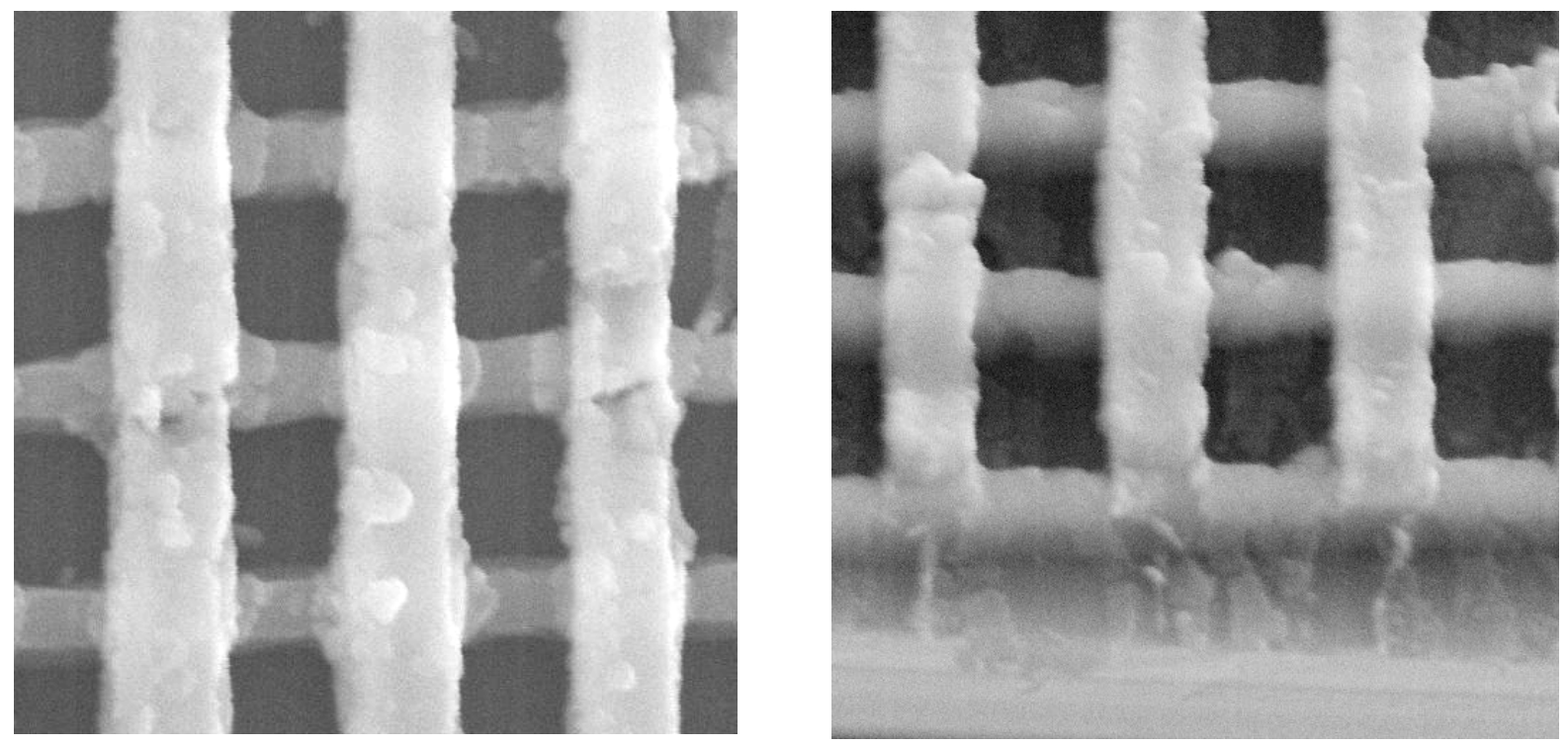

Figure 4.17 - Two-layer slurry-infiltrated titania structure. The structure has a periodicity of $2.5 \mu \mathrm{m}$. The image on the right is the tilted view of the structure.

So far the titania slurries were only used to infiltrate epoxy molds with periodicity of 2.5 micron (which have a gap width of about 1.5 micron). In order to be able to infiltrate the epoxy molds with periodicity of 1 micron (which have a bar spacing of about 0.6 micron) or smaller, the smaller size of the titania particles as well as the stability of the particles in the solution becomes more critical. 


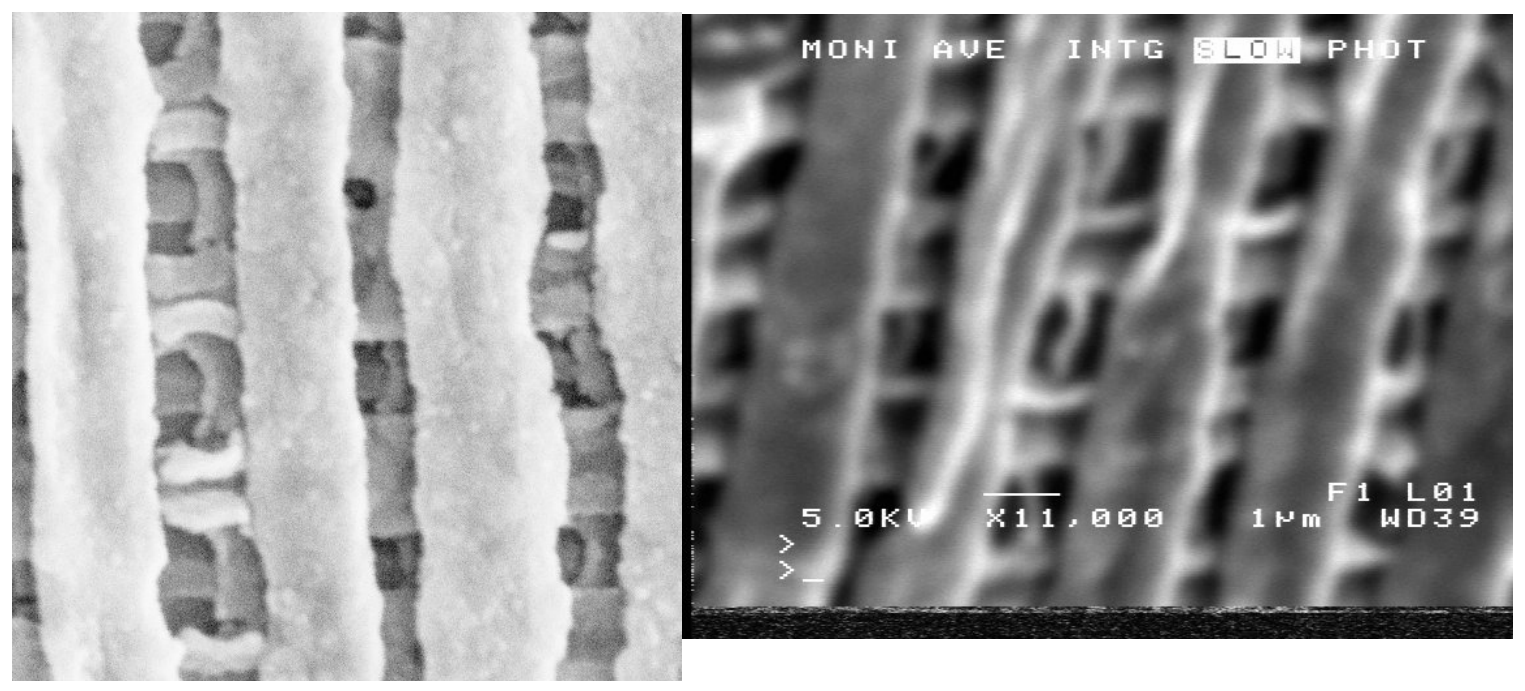

Figure 4.18 - Four-layer slurry-infiltrated titania structure. The structure has a periodicity of $2.5 \mu \mathrm{m}$. The image on the right is the tilted view of the structure.

\section{Sol Gel Infiltration}

Henry Kang provided the following results. TDBP was diluted with isopropanol from its original weight percent of $75 \%$ to $25 \%$ for infiltrating two-layer epoxy molds. A two-layer epoxy mold with periodicity of 2.5 micron was spin coated with diluted TDBP at $4000 \mathrm{rpm}$ for 1 minute, then placed in a humidity chamber for $\sim 24$ hours to promote the gelation reaction. The process was repeated to ensure the epoxy mold was properly filled. The resulting structure after calcination is shown in Figure 4.19. Compared to samples infiltrated by titania slurries, the samples resulted from the sol-gel processing better represented the inverse structure of the epoxy molds. However, the titania bars at the top layer tended to sag downward in between each two supporting bars of the bottom layer, as shown in Figure 4.19. The main cause of this phenomenon has not been well understood, however, it is possible to improve the sagging by applying more precursor materials. A fourlayer epoxy mold was spin coated with TDBP as received (75 wt\%) at $5000 \mathrm{rpm}$ for a minute. The infiltrated sample was then dried in air for about three days. The resulting structure after heat treatment is shown below in Figure 4.20. Again, the four-layer sample prepared by sol-gel processing exhibited a better structure than the ones prepared by titania slurry. However, the sagging phenomenon described above was still observed. 

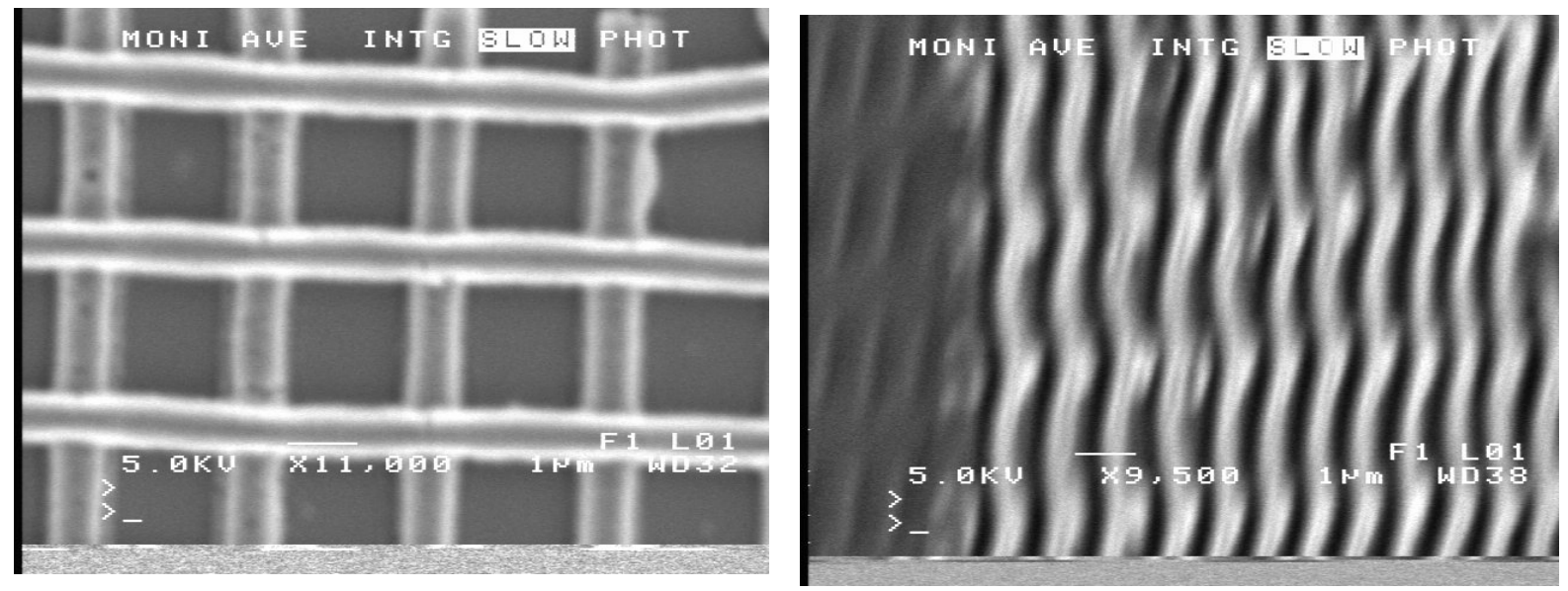

Figure 4.19 - Two-layer TDBP infiltrated titania structure. The structure has a periodicity of $2.5 \mu \mathrm{m}$. The image on the right is the tilted view of the structure taken at a tilt angle of $\sim 80^{\circ}$. Notice the sagging phenomenon of the titania bars.
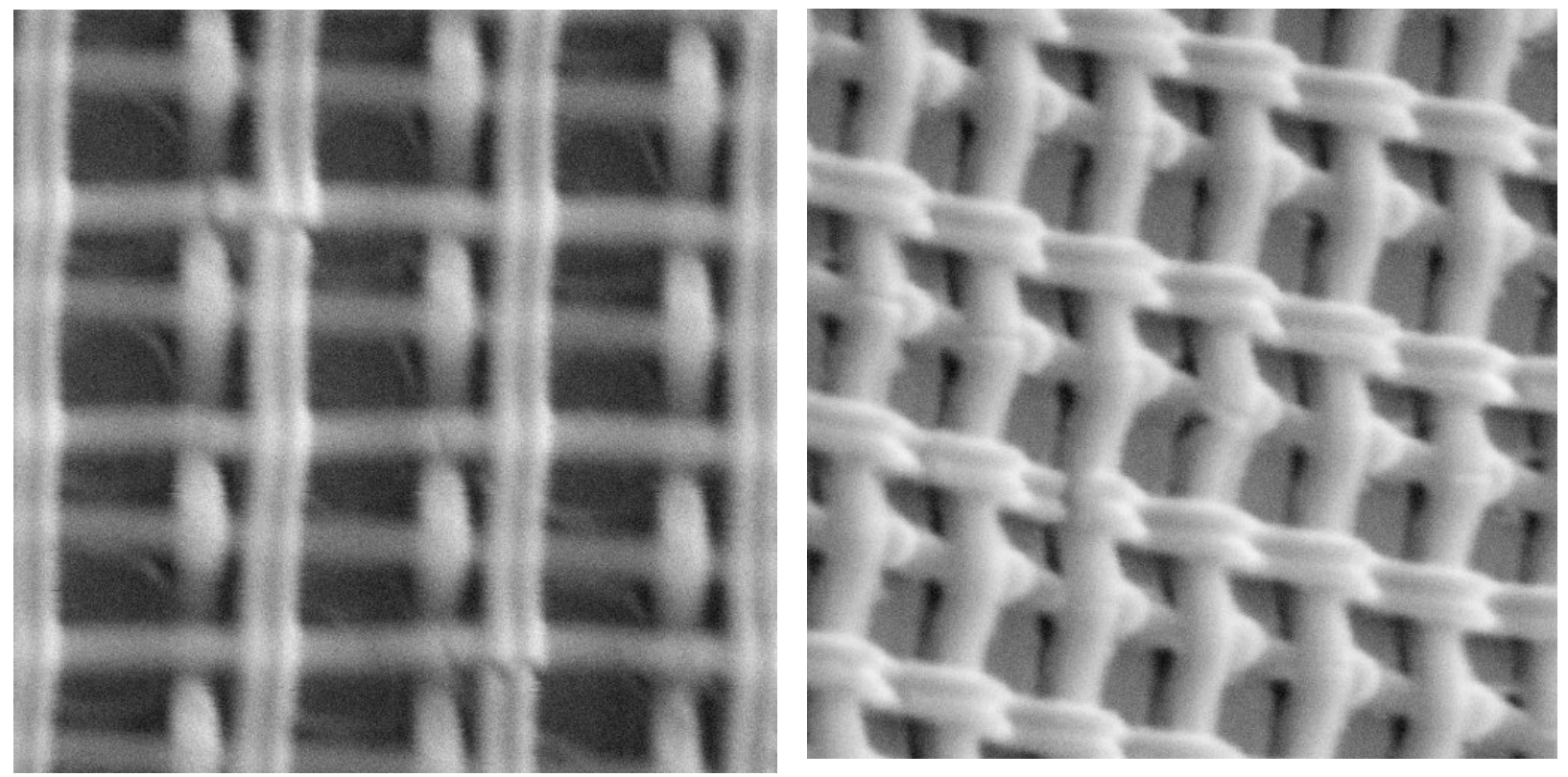

Figure 4.20 - Four-layer TDBP infiltrated titania structure. The structure has a periodicity of $2.5 \mu \mathrm{m}$. The image on the right is the tilted view of the structure taken at a tilt angle of $\sim 60^{\circ}$. Notice the sagging phenomenon of the titania bars. 
An attempt to solve the sagging problem observed in the 2.5 micron sample was to use TIPP instead of TDBP. The preliminary study indicated that the TIPP precursor would leave a larger amount of titania, which could lead to less sagging after heat treatment. However, 2.5 micron samples prepared by TIPP precursor generally exhibited a broken network.

A trial of spin coating a two-layer 1.0 micron periodicity sample with TIPP resulted in a good structure. The spin coating process was performed in a dry argon glove box with a spinning speed of $2000 \mathrm{rpm}$ and a spinning time of one minute. The sample was left in the glove box for a day and then was exposed to air to allow TIPP to gel. The resulting structure after heat treatment is shown in Figure 4.21. Also, the sagging phenomenon was not as evident in the 1.0 micron sample. (Figure 4.22) This result may imply that the sagging will not be an issue for even smaller dimension of molds.
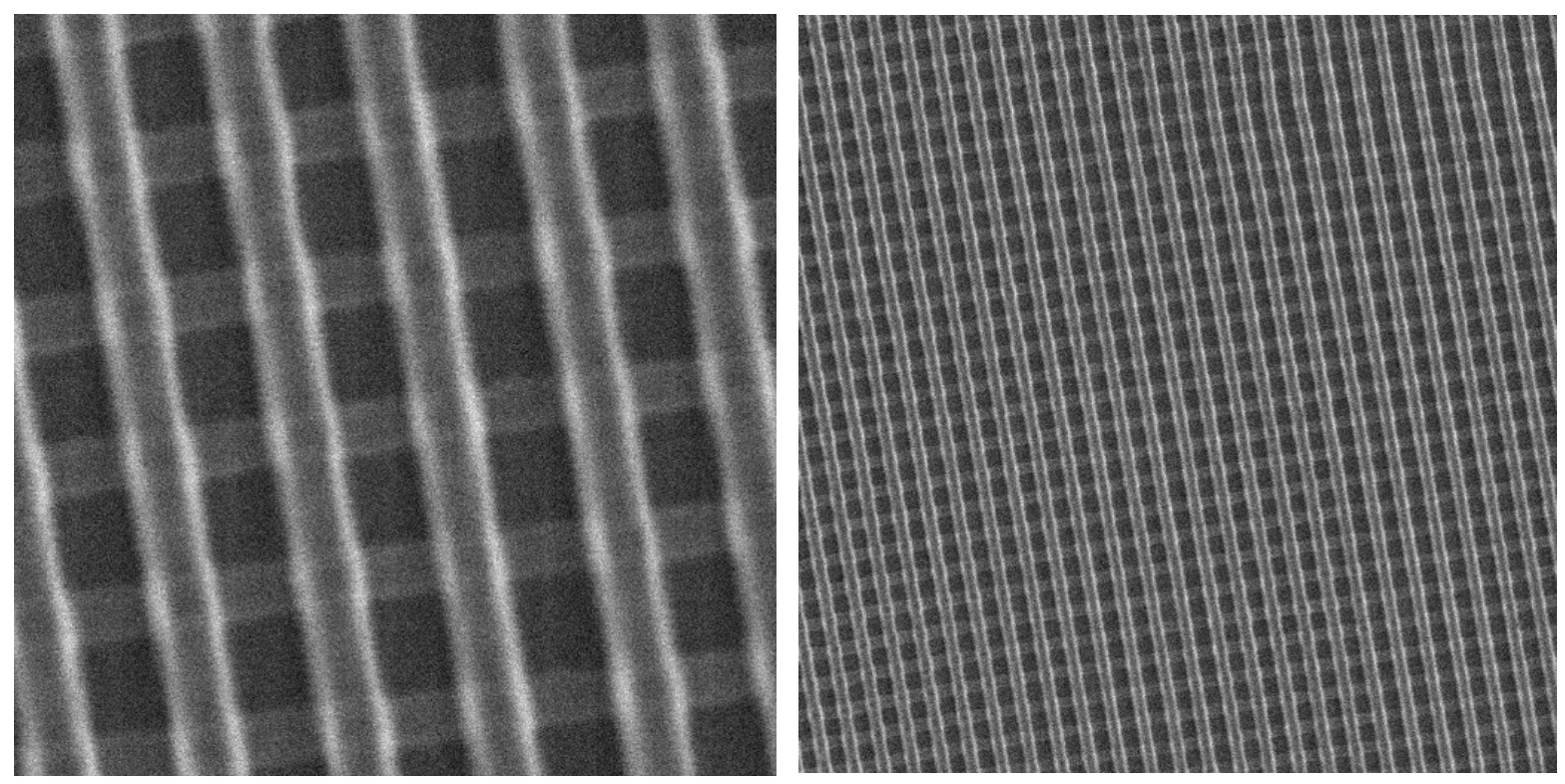

Figure 4.21 - Two-layer TIPP infiltrated titania structure. The structure has a periodicity of $1.0 \mu \mathrm{m}$. 


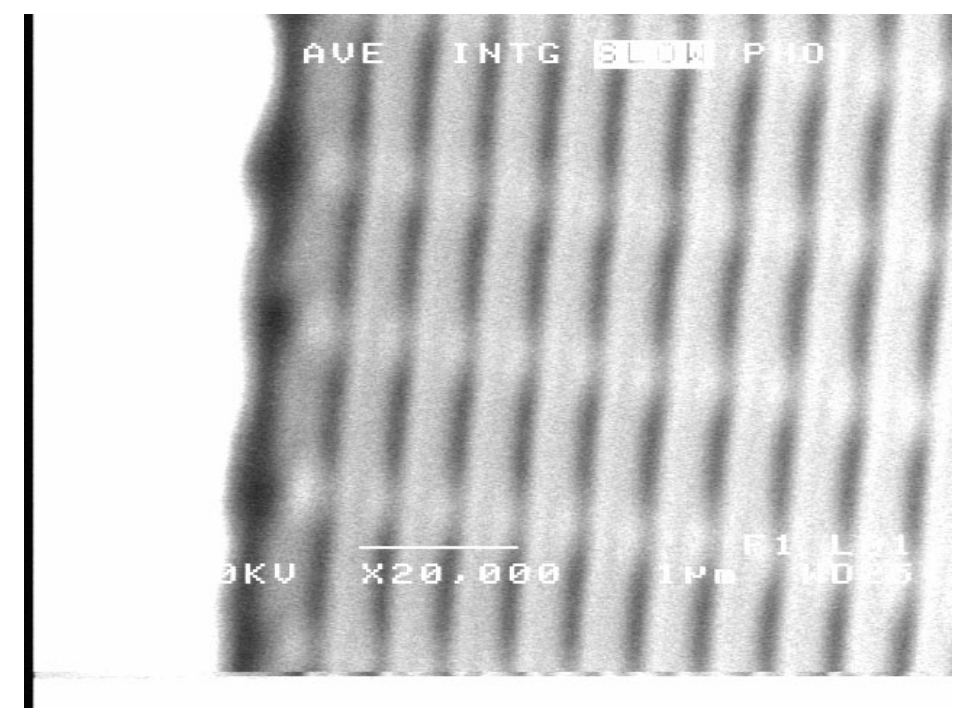

Figure 4.22 - The sagging phenomenon was not obvious in the sample with a periodicity of 1.0 micron. The image was taken at a tilted angle of $\sim 80^{\circ}$.

A four-layer 1.0 micron sample was also fabricated with similar exceptional results. It was made with the same procedure as the two-layer sample above, except for a different spin rate of $1000 \mathrm{rpm}$. The structure is shown in figure 4.23 .

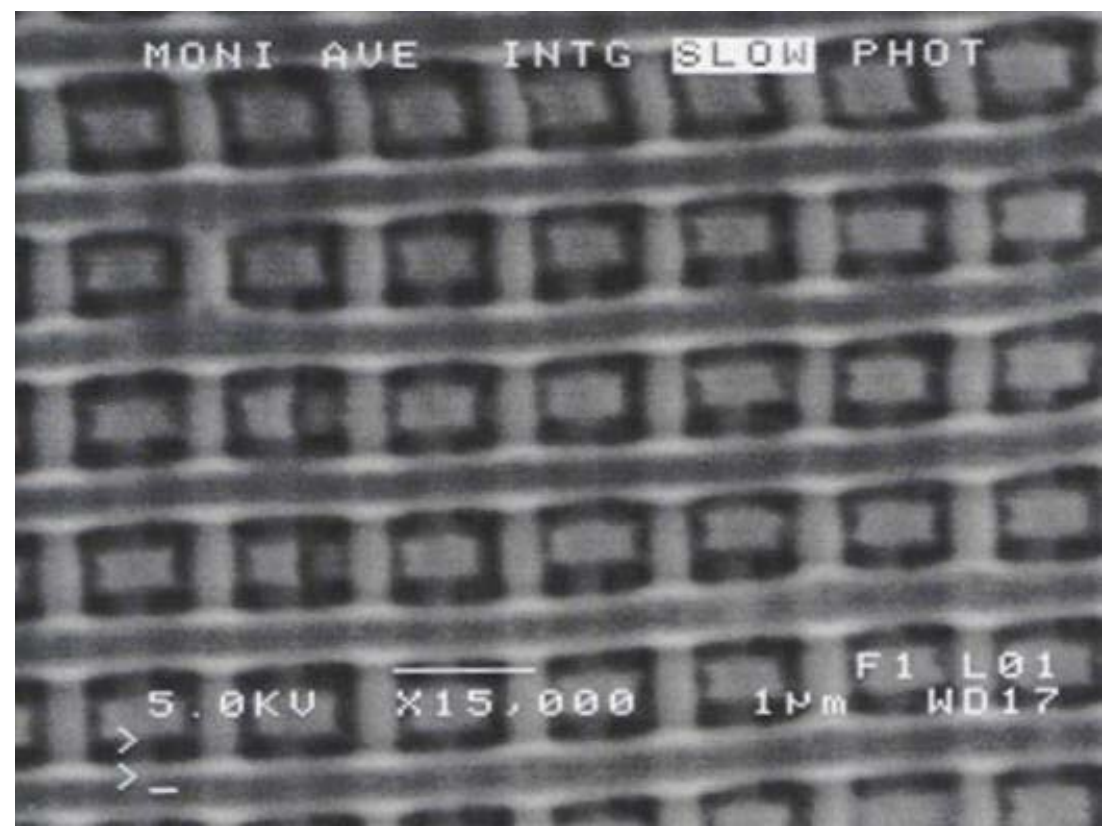

Figure 4.23 - A four-layer 1.0 micron sample infiltrated with a sol-gel process 
The slurry and sol-gel infiltrated titania samples are a direct result of the quality of the original epoxy mold used in their fabrication. Usually, the higher quality epoxy molds were used for infiltration purposes, which is a main reason for the success of the titania structures.

\section{Other Analysis Methods}

The PBG structure integrity was also tested with equipment built by Chan-Hwan Kim from the Iowa State physics department. The reflected diffraction pattern from the mold was captured by a CCD camera to be analyzed. The reflected pattern from a 2.5 micron sample is shown in figure 4.24. The sample is a two-layer structure, and one can see that the first layer is good but the second layer is bad. This is evident because the diffraction spots are clear and distinct in the direction perpendicular to the first layer (horizontally, and there are hazy lines in between the vertical diffraction spots that correspond to the second layer. This is because the defects cause several diffraction conditions to be satisfied, creating various intensities of the light along a line.

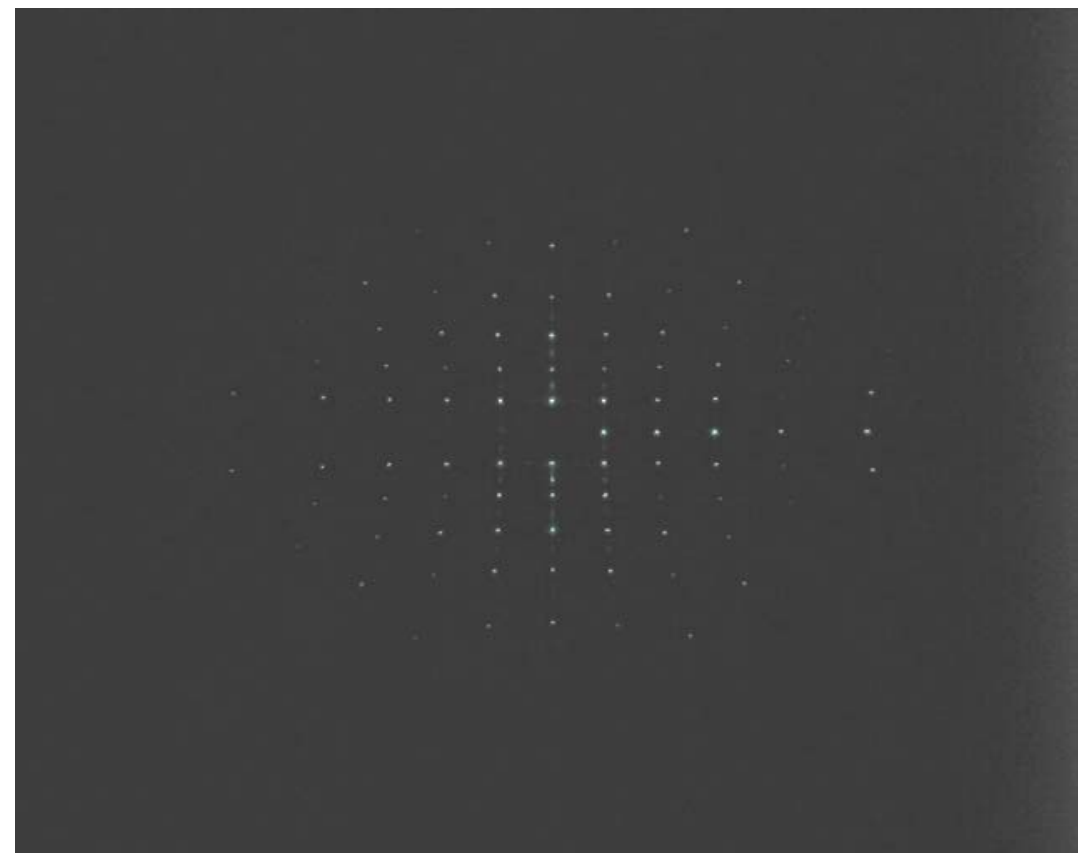

Figure 4.24 - The reflected diffraction pattern from a 2.5 micron epoxy bar sample illuminated with a $457 \mathrm{~nm}$ laser 


\section{Conclusions}

The main conclusion drawn from this research is that there is no ideal process that can be used consistently to make good epoxy layered structures with the current procedure. To be able to develop an optimal procedure, an entirely mechanized process would be necessary in a controlled environment. With such a system, a design of experiments could be performed to optimize all of the variables involved.

Without a mechanized procedure, it is necessary to discuss the effect of each variable, which has been summarized previously in table 4.1. The parameter that has the most effect on the final epoxy structure and is also one of the easier variables to control and quantify is the number of times the epoxy is swept back and forth across the mold. The phrase "Swept back and forth" signifies that the epoxy was moved from one end of the pattern in a side-toside motion and back. It was found, depending on several other variables, that the optimal number of sweeps was in the range of 9-20 sweeps for the 2.5 micron samples and in the range of 7-14 sweeps for the 1.0 micron samples. The best observed values for the other variables are summarized in table 4.7.

Table 4.7 - Optimal ranges for the variables in the infiltration process

\begin{tabular}{|c|c|}
\hline Variable & Optimal Range \\
\hline Viscosity of Epoxy (Two Choices) & 180 or $250 \mathrm{cps}$ \\
\hline PDMS Furnace Time & 5 hrs to 2 days \\
\hline Particle Contamination & none \\
\hline PDMS Resin to Hardener Ratio & 10 to 1 \\
\hline Time Between Furnace Holds & $5-10$ minutes \\
\hline Time Between Application of Newly Produced Layer & $3-4$ hours \\
\hline Sweep Speed & depends \\
\hline Number of Sweeps Between Furnace Holds & depends \\
\hline Furnace Temperature & $60-70$ oC \\
\hline Ambient Temperature & $65-75$ oC \\
\hline Humidity & low as possible \\
\hline Number of Total Sweeps & depends \\
\hline Epoxy resin to hardener ratio & $2.7-3.3$ to 1 \\
\hline Aemperature Change of Sample During Epoxy & Hot for infiltration but cold for \\
epoxy adhesion
\end{tabular}


Observations were also made concerning the dependence of various aspects of the infiltration process. Figures 4.25 through 4.27 show schematic drawings of the dependence of temperature on the viscosity of the epoxy, the curing time of the epoxy, and the expansion of the epoxy respectively. Figure 4.28 is a schematic drawing of dependence of curing on the volume of the epoxy.

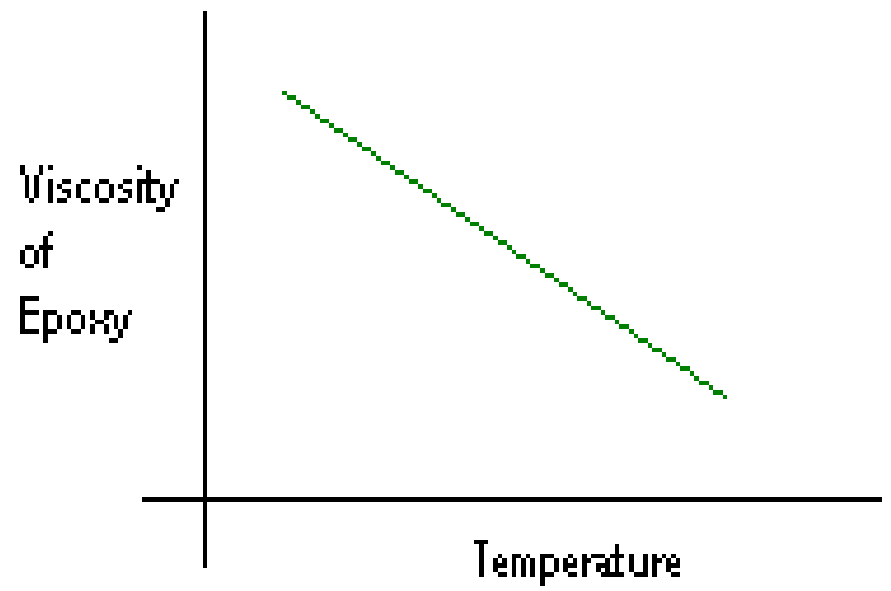

Figure 4.25 - Schematic sketch of the dependence of viscosity on temperature of epoxy

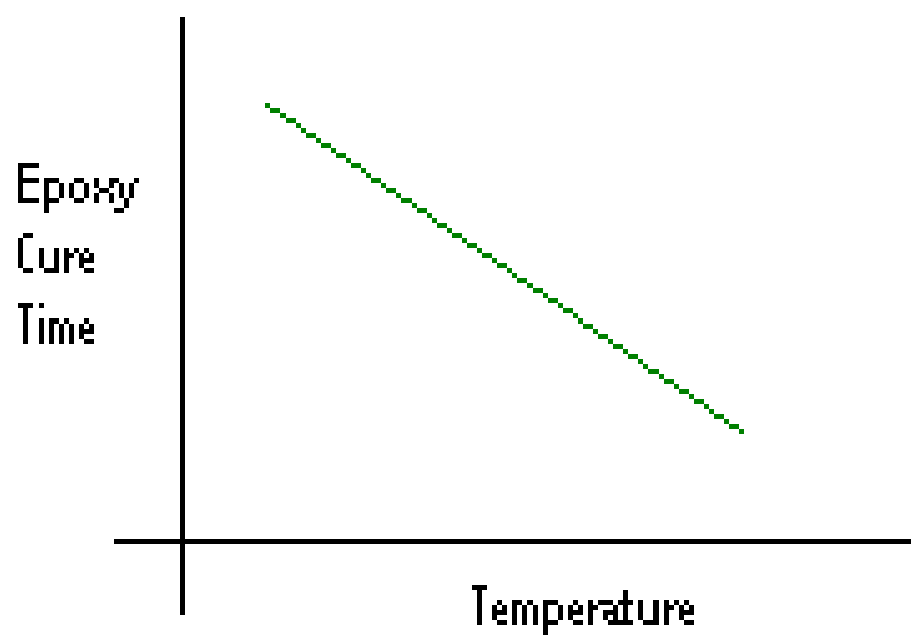

Figure 4.26 - Schematic sketch of the dependence of epoxy cure time on temperature 


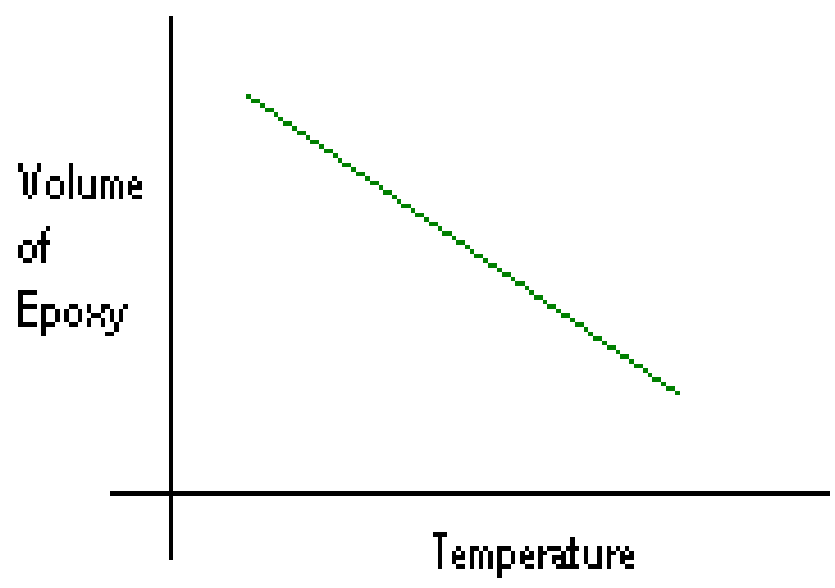

Figure 4.27 - Schematic sketch of thermal expansion characteristic of uncured epoxy

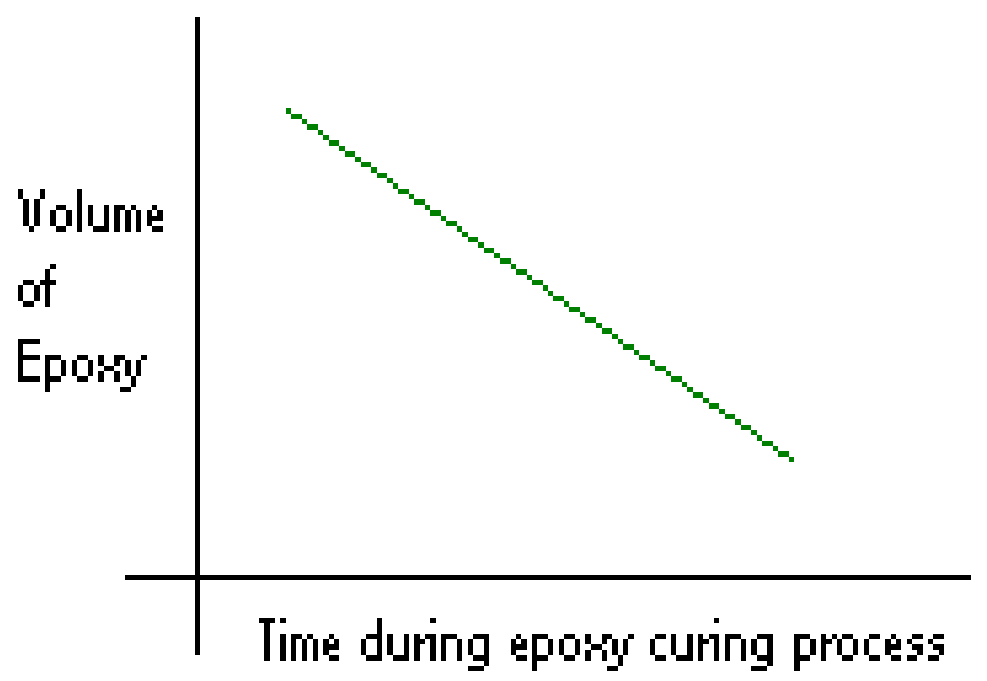

Figure 4.28 - Schematic sketch of the expansion characteristic of curing process

These rough graphs are used to explain why the infiltration process is difficult. The infiltration must take place in a hot environment, but the sample must be cooled so that the viscosity of the epoxy is high enough that it remains in the grooves. The epoxy appears to expand at this point, causing the mold to be overfilled. As the epoxy cures, it contracts, using the overfilled reserves then leaving defects if the volume of epoxy present is not adequate. Therefore, it is common for molds to be either overfilled or entirely filled with defects by the time they are needed for a subsequent layer application. 
It was also concluded that the alignment step of the process needs to be drastically improved before samples exhibit excellent third-layer alignment across their entire surface area. No available alignment system is capable of placing the third layer correctly, which is limited by the flexibility of the PDMS mold. The 2.5 micron samples were the only samples that could be used in the laser alignment fabricated for this research. A shorter wavelength laser would be necessary to make the system functional for the 1.0 micron samples.

A final conclusion drawn from the research is that the infiltration process generally became more simple and consistent as sample dimensions were reduced. This was not the case for the 0.4 micron samples, but these problems can be attributed to master mold defects. This trend is expected to continue for smaller and smaller mold dimensions until a critical groove size is reached. Titania samples fabricated from the slurry infiltration method will reach this critical dimension much earlier because of the large particle size of the slurry compared to the groove width. 


\section{CHAPTER 5: FUTURE WORK}

\section{Micro-Transfer Molding}

The original objective of this research was to develop a photonic band structure and analyze that structure with laser dye emission spectrum techniques. This was not achieved with the current procedure in this time frame, and there are several accomplishments that need to be realized before this objective is possible with the $\mu \mathrm{TM}$ technique:

1. A master mold with a periodicity around $300 \mathrm{~nm}$

2. A procedure to infiltrate channels of that size

3. A method that can be used to precisely align up to at least four layers

4. An infiltration process that works for that size.

The master mold problem cannot be solved with the semiconductor processing technology available at Iowa State University. It could be solved by ordering a silicon mold from a company with the necessary processing techniques. Wai Leung is currently working on this problem.

The ability to consistently infiltrate a smaller sample with the current epoxy infiltration process used should be easier because of the lessened necessary volume of epoxy necessary to fill the pattern and the increased capillary action of the narrower grooves. This was the trend in moving from the 2.5 micron sample to the 1.0 micron sample, but this trend was disrupted by the attempt at fabricating a 0.4 micron sample. The causes of the problems with this sample were most likely defects and imperfections in the silicon master mold. Even the one-drop $1^{\text {st }}$ layer method of infiltrating the 0.4 micron pattern, which had always been successful for other molds, produced extremely poor results. This suggests that the grooves in the PDMS were not continuous or even existent at certain places on the pattern. Whether or not this was a problem caused by the silicon pattern or the PDMS layer needs to be examined further. More in depth analysis could also be performed on the surface effects and capillary action forces that affect the ability to apply epoxy to the smaller grooves. It was 
also suggested that the mixing of the epoxy might not have been complete to the dimensions of the 0.4 micron molds. More investigation is necessary to determine if this is a factor in the infiltration process. Another suggestion made to improve infiltration is the use of a UV curable epoxy, which would eliminate all of the problems associated with the heating and cooling of the samples.

Alignment is a big concern as the bar periodicity is reduced to below 0.4 microns. One problem with the smaller patterns is that a diffraction pattern can no longer be used to align the samples since the condition for diffraction is no longer satisfied for wavelengths greater than the bar spacing. Proposed solutions to this problem include utilizing a UV radiation and detection system or including an alignment region outside of the smaller patterned area. The latter of these solutions would be more feasible. It would require that the alignment region be implemented into the silicon master design for the pattern. Wider spaced bars that correlated to the periodicity of the smaller region would be used to be able to align the $1^{\text {st }}$ and $2^{\text {nd }}$ layers easily. The alignment of the third layer becomes much more difficult. The previously mentioned error because of the flexibility of the PDMS molds makes this a very difficult step to accomplish. Without even addressing the PDMS problem, the laser alignment system currently used is not capable of third layer alignment. A more complicated design needs to be developed that will allow extremely close movement of the two layers without contact. If this is possible, lasers could be utilized to perform third and forth layer alignments. Experimentation with the optical alignment method could also result in success for the smaller periodicity samples.

Lastly, the titania infiltration process will need to be perfected for the smaller samples. Similarly to the epoxy infiltration, it is thought that the results will be much better for smaller and smaller bar sizes. The results from the 1.0 micron samples in this case were amazingly better than the previous results from the 2.5 micron samples. This trend is hoped to continue for the smaller samples. The problem still exists that the thickness of the sol-gel samples is minimal, and also the four layer samples underwent severe cracking because of the shrinkage during the firing process. These problems could be solved by a slurry and solgel concurrent infiltration. The slurry would provide a greater amount of material that would 
undergo less shrinkage during firing, and the sol-gel would infiltrate into the small dimensions not reached by the larger titania slurry particles.

\section{Laser Dye Analysis}

Laser dye luminescence was the original method for this research intended to analyze the PBG structures. There are several possible laser dyes that could be used to infiltrate the PBG samples in an attempt to discover low threshold, high intensity lasing properties. The future work intended, however, to fabricate an LED out of the final PBG structure, utilizing the polymer laser dye PPV. The PPV was to be used because of its property of electroluminescence. The proposed construction of the LED is shown in figure 5.1. The glass substrate is originally coated with an indium-tin oxide (ITO) electrode and a small portion is etched from one side. The 3-D PBG structure is built on top of the ITO and then the PPV polymer is spin coated on through the structure. After the PPV laser dye polymer is set, an aluminum electrode can then be applied to the top as shown in the figure, ensuring that the two electrodes do not form an electrical contact. Using this device, the PBG structure can be evaluated by its effect on the spectral intensities of the PPV. The normal luminescence spectrum for electrically pumped PPV ranges from around $500 \mathrm{~nm}$ to around $700 \mathrm{~nm}$ with a luminescence peak at around $550 \mathrm{~nm}$. The PBG structures inset into the LED devices constructed will cause a dip in that spectrum along with a photonic band edge lasing phenomenon. The spectrum is expected to display a high intensity, small linewidth peak at the photonic band edge because of the reflective effects occurring within the structure. A normal luminescence spectrum is expected to be emitted through the bottom of the LED structure, which also can be measured. A diagram showing the expected luminescence plots for each case is shown in figure 5.2. 


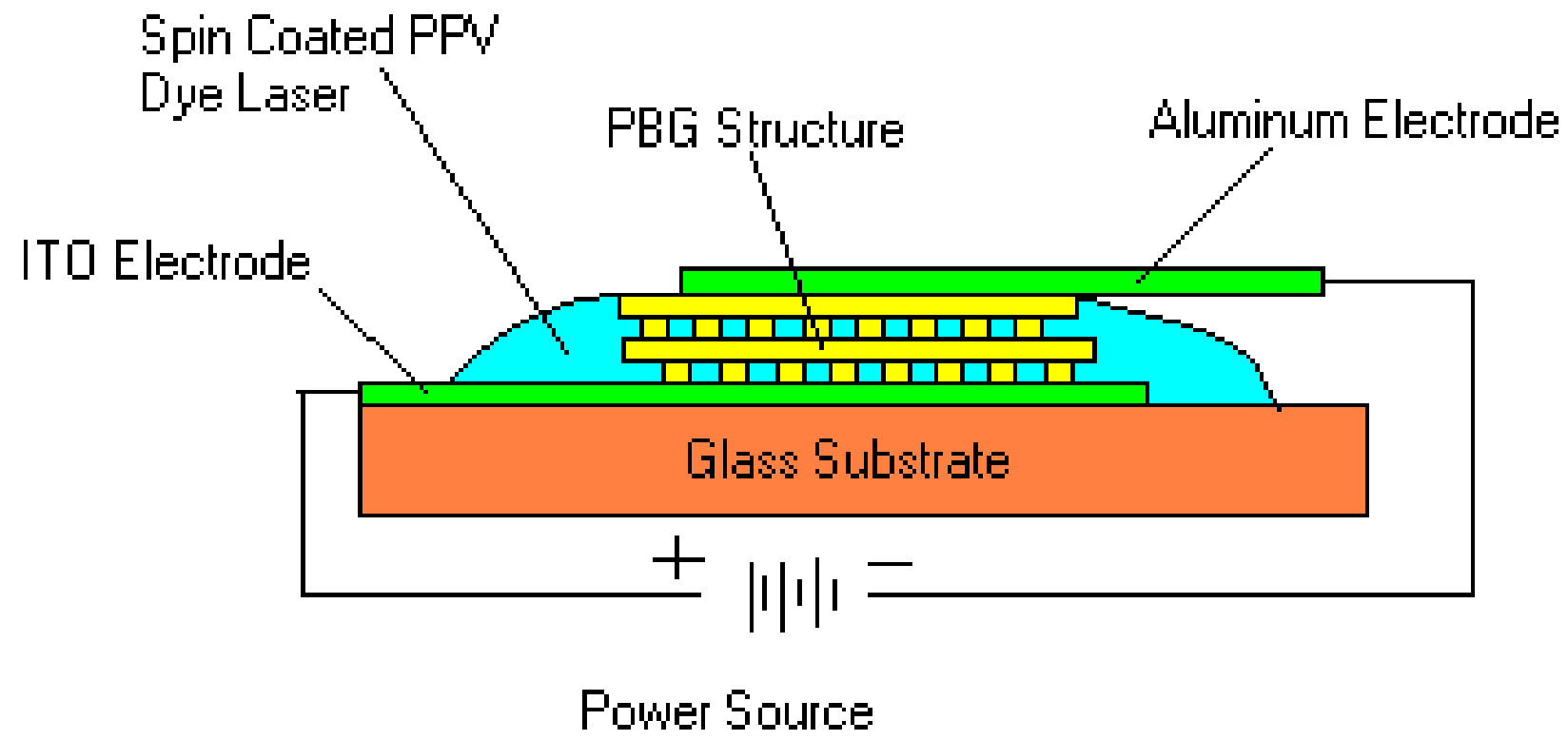

Figure 5.1 - LED Device construction
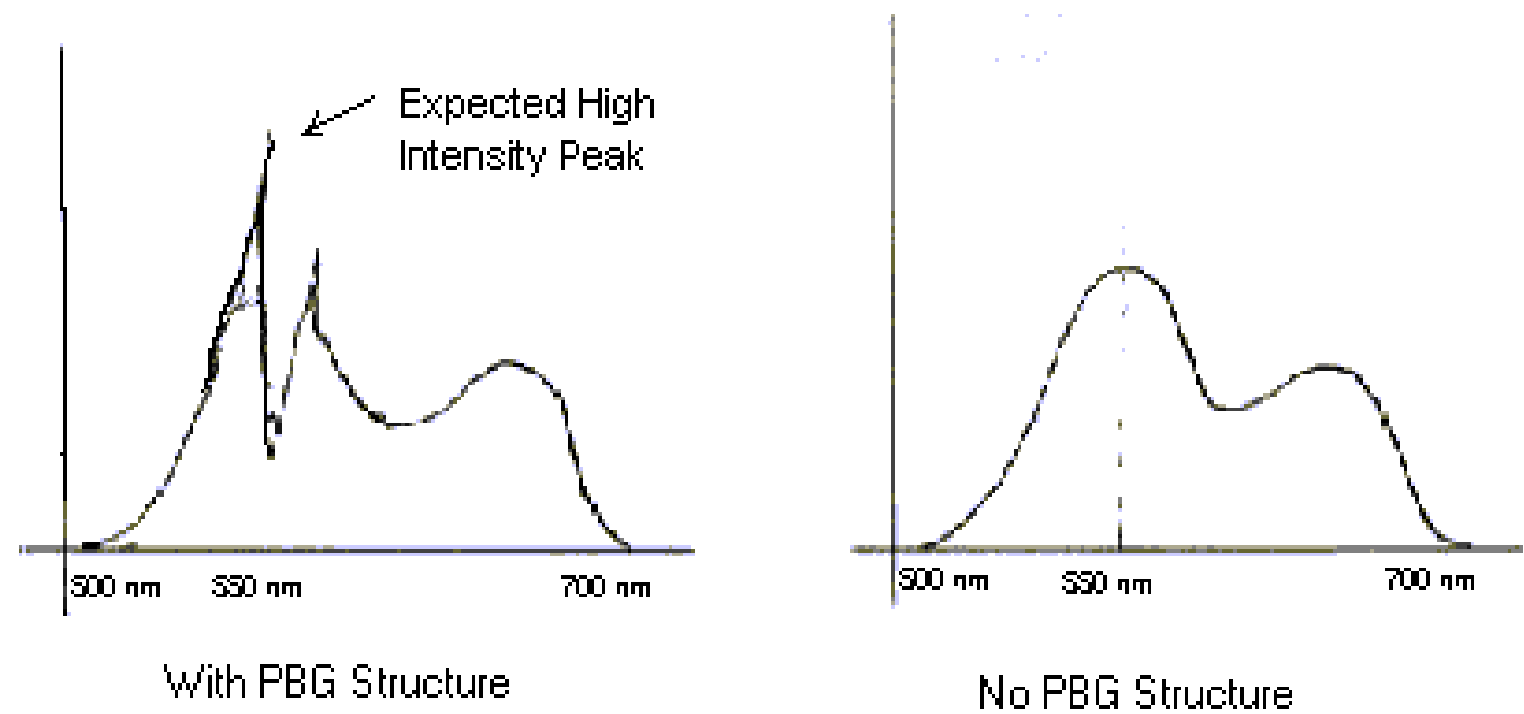

No PBG Structure

Figure 5.2 - The expected luminescence spectrum for emission through the PBG structure with a band gap around $550 \mathrm{~nm}$ and a common PPV spectrum of luminescence emission. 


\section{Summary of Future Work}

Below is a list summarizing the points made in the Future Work section of this paper.

- Order a new master silicon mold at a periodicity range less than 0.3 microns

- Develop a procedure that can be used to infiltrate the smaller grooves, which may involve researching surface energy effects and finding new materials

- Investigate the extent of the mixing of the epoxy

- Look into ultra-violet curing epoxies

- Design an alignment method capable of aligning the first and third layers - could optimize optical alignment procedure or design new laser alignment equipment

- Investigate the alignment problem caused by the PDMS mismatch

- Develop a procedure to infiltrate the smaller epoxy molds with sol-gel

- Design and use an electrically pumped LED to characterize the final structure 


\section{REFERENCES}

\section{General PBG}

1. E Yablonovitch. "Inhibited Spontaneous Emission in Solid-State Physics and Electronics.” Physical Review Letters. 58, 2059 (1987)

2. S. John. "Strong Localization of Photons in Certain Disordered Dielectric Superlattices.” Physical Review Letters. 58, 2486 (1987)

3. E. Yablonovitch and T. J. Gmitter. "Photonic Band Structure: The Face-CenteredCubic Case.” Physical Review Letters. 63, 1950 (1989)

4. K. M. Ho, C. T. Chan, and C. M. Soukoulis. Existence of a Photonic Gap in Periodic Dielectric Structures.” Physical Review Letters. 65, 3152 (1990)

5. E. Yablonovitch. "Photonic Band Structure." Analogies in Optics and Microelectronics. 117-133 (1990)

6. E. Yablonovitch and T. J. Gmitter. "Photonic Band Structure: The Face-CenteredCubic Case Employing Nonspherical Atoms.” Physical Review Letters. 67, 2295 (1991)

7. E. Yablonovitch. "Photonic Band-Gap Structures." Journal of the Optical Society of America B. 10, 283 (1993)

8. A. A. Maradudin and A. R. McGurn. "Photonic Band Structure of a Truncated, 2-D Periodic Dielectric Medium." Journal of the Optical Society of America B. 10, 307 (1993)

9. W. M. Robertson, G. Arjavalingam, R. D. Meade, K. D. Brommer, A. M. Rappe, and J. D. Joannopoulos. "Measurement of the Photon Dispersion Relation in 2-D Ordered Dielectric Arrays." Journal of the Optical Society of America B. 10, 322 (1993)

10. R. D. Meade, A. M. Rappe, K. D. Brommer, and J. D. Joannopoulos. "Nature of the Photonic Band Gap: Some Insights from a Field Analysis." Journal of the Optical Society of America B. 10, 328 (1993)

11. T. K. Gaylord, G. N. Henderson, and E. N. Glytsis. "Application of Electromagnetics Formalism to Quantum-Mechanical Electron-Wave Propagation in Semiconductors." Journal of the Optical Society of America B. 10, 333 (1993)

12. J. P. Dowling and C. M. Bowden. "Beat Radiation from Dipoles near a Photonic Band Edge." Journal of the Optical Society of America B. 10, 353 (1993) 
13. G. Taubes. "Photonic Crystal Made to Work at an Optical Wavelength." Science. 278, 1709 (1997)

14. H.-B. Lin, R. J. Tonucci, and A. J. Campillo. "2-D Photonic Band gap Optical Limiter in the Visible." Optics Letters. 23, 94 (1998)

15. S. Rowson, A. Chelnokov, J.-M. Lourtioz, and F. Carcenac. "Reflection and Transmission Characterization of a Hexagonal Photonic Crystal in the Mid Infrared." Journal of Applied Physics. 83, 5061 (1998)

16. J. C. Knight, J. Broeng, T. A. Birks, P. St. J. Russell. "Photonic Band Gap Guidance in Optical Fibers." Science 282, 1476 (1998)

17. K. Sakoda, M. Sasada, T. Fukushima, A. Yamanaka, N. Kawai, and K. Inoue.

"Detailed Analysis of Transmission Spectra and Bragg-reflection Spectra of a 2-D Photonic Crystal with a Lattice Constant of $1.15 \mu \mathrm{m}$." Journal of the Optical Society of America B. 16, 361 (1999)

18. A. Chutinan and S. Noda. "Effects of Structural Fluctuations on the Photonic Band gap During Fabrication of a Photonic Crystal: a Study of a Photonic Crystal with a Finite Number of Periods." Journal of the Optical Society of America B 16, 1398 (1999)

19. K. Sakoda, M. Sasada, T. Fukushima, A. Yamanaka, N. Kawai, and K. Inoue.

"Detailed Analysis of Transmission Spectra and Bragg-Reflection Spectra of a 2-D

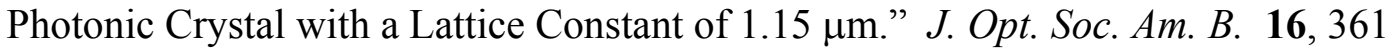
(1999)

20. S. W. Leonard, H. M. Van Driel, K. Busch, S. John, A. Birner, A.-P. Li, F. Muller, U. Gosele, and V. Lehmann. "Attenuation of Optical Transmission Within the Band Gap of Thin 2-D Macroporous Silicon Photonic Crystals." Applied Physics Letters. 75, 3063 (1999)

21. M. Doosje, B. J. Hoenders, and J. Knoester. "Photonic Band gap Optimization in Inverted fcc Photonic Crystals.” J. Opt. Soc. Am. B. 17, 600 (2000)

PBG-Layered Production Method

22. K. M. Ho, C. T. Chan, C. M. Soukoulis, R. Biswas, and M. Sigalas. "Photonic Band Gaps in 3-D: New Layer-By-Layer Periodic Structures." Solid State

Communications. 89, 413 (1994)

23. E. Ozbay, E. Michel, G. Tuttle, R. Biswas, M. Sigalas, and K.-M. Ho. "Micromachined millimeter-wave photonic band-gap crystals." Applied Physics Letters. 64, 2059 (1994) 
24. E. Ozbay, A. Abeyta, G. Tuttle, M. Tringides, R. Biswas, C. T. Chan, C. M. Soukoulis, and K.-M. Ho. "Measurement of a 3-D photonic band gap in a crystal structure made of dielectric rods." Physical Review B. 50, 1945 (1994)

25. S. Noda, N. Yamamoto, and A. Sasaki. "New Realization Method for 3-D Photonic Crystal in Optical Wavelength Region." Japanese Journal of Applied Physics. 35, L909 (1996)

26. E. Ozbay. "Layer-by-layer Photonic Crystals From Microwave to Far-infrared Frequencies." Journal of the Optical Society of America B. 13, 1945 (1996)

27. R. Biswas, E. Ozbay, and K.-M. Ho. "Photonic Band Gaps with Layer-by-Layer Double-Etched Structures." Journal of Applied Physics. 80, 6749 (1996)

28. M. C. Wanke, O. Lehmann, K. Muller, Q. Wen, and M. Stuke. "Laser Rapid Prototyping of Photonic Band Gap Microstructures." Science. 275, 1284 (1997)

29. N. Katsarakis, E. Chatzitheodoridis, G. Kiriakidis, M. M. Sigalas, C. M. Soukoulis, W. Y. Leung, and G. Tuttle. "Laser-machined Layer-by-layer Metallic Photonic Bandgap Structures." Applied Physics Letters. 74, 3263 (1999)

30. S. Noda, N. Yamamoto, M. Imada, H. Kobayashi, and M. Okano. "Alignment and Stacking of Semiconductor Photonic Band gaps by Wafer-Fusion.” Journal of Lightwave Technology. 17, 1948 (1999)

31. S. Y. Lin, J. G. Fleming, D. L. Hetherington, B. K. Smith, R. Biswas, K. M. Ho, M. M. Sigalas, W. Zubrzycki, S. R. Kurtz, and Jim Bur. "A Three-Dimensional Photonic Crystal Operating at Infrared Wavelengths." Nature. 394, 251 (1999)

32. A. Feigel, Z. Kotler, B. Sfez, A. Arsh, M. Klebanov, and V. Lyubin. "Chalcogenide Glass-based Three-Dimensional Photonic Crystals." Applied Physics Letters. 77, 3221 (2000)

PBG-Graphite Structure

33. F. Gadot, A. Chelnokov, A. De Lustrac, P. Crozat, and J.-M. Lourtioz. "Experimental Demonstration of Complete Photonic Band Gap in Graphite Structure." Applied Physics Letters. 71, 1780 (1997)

34. A. A. Zakhidov, R. H. Baughman, Z. Iqbal, C. Cui, I. Khayrullin, S. O. Dantas, J. Marti, and V. G. Ralchenko. "Carbon Structures with 3-D Periodicity at Optical Wavelengths." Science. 282, 897 (1998) 
PBG-Lithography Production

35. P. L. Gourley, J. R. Wendt, G. A. Vawter, T. M. Brennan, and B. E. Hammons. "Optical Properties of 2-D Photonic Lattices Fabricated as Honeycomb Nanostructures in Compound Semiconductors." Applied Physics Letters. Sandia National Lab (1993)

36. V. V. Poborchii, T. Tada, and T Kanayama. "A Visible-Near Infrared Range Photonic Crystal Made up of Si Nanopillars.” Applied Physics Letters. 75, 3276 (1999)

37. M. Golosovsky, Y. Saado, and D. Davidov. "Self-assembly of Floating Magnetic Particles into Ordered Structures: A Promising Route for the Fabrication of Tuanble Photonic Band Gap Materials.” Applied Physics Letters. 75, 4168 (1999)

38. M. Campbell, D. N. Sharp, M. T. Harrison, R. G. Denning, and A. J. Turberfield. "Fabrication of Photonic Crystals for the Visible Spectrum by Holographic Lithography." Nature. 404, 53 (2000)

PBG-Simple Cubic Lattice Structures

39. H. S. Sozuer, and J. W. Haus. "Photonic Bands: Simple-Cubic Lattice." Journal of the Optical Society of America B. 10, 296 (1993)

40. M. Wada, Y. Doi, K. Inoue, J. W. Haus, and Z. Yuan. "A simple-cubic Photonic Lattice in Silicon." Applied Physics Letters. 70, 2966 (1997)

41. L. Zavieh, and T. S. Mayer. "Demonstration of a 3D Simple-cubic Infrared Photonic Crystal.” Applied Physics Letters. 75, 2533 (1999)

PBG-Fluorescence

42. T. W. Mossberg and M. Lewenstein. "Radiative Properties of Strongly Driven Atoms in the Presence of Photonic Bands and Gaps." Journal of the Optical Society of America B. 10, 340 (1993)

43. B. Y. Tong, P. K. John, Y. Zhu, Y. S. Liu, S. K. Wong, and W. R. Ware.

"Fluorescence-Lifetime Measurements in Monodispersed Suspensions of Polystyrene Particles." Journal of the Optical Society of America B. 10, 356 (1993)

PBG-Defect Modes

44. K. M. Leung. "Defect Modes in Photonic Band Structures: A Green's Function Approach Using Vector Wannier Functions." Journal of the Optical Society of America B. 10, 303 (1993) 
45. D. R. Smith, R. Dalichaouch, N. Kroll, S. Schultz, S. L. McCall, and P. M. Platzman. "Photonic Band Structure and Defects in one and two Dimensions." Journal of the Optical Society of America B. 10, 314 (1993)

46. G. Kurizki, B. Sherman, and A. Kadyshevitch. "Quantum Electrodynamics in Photonic Band Gaps: Atomic-Beam Interaction with a Defect Mode." Journal of the Optical Society of America B. 10, 346 (1993)

PBG-Opal Structures

47. S. G. Romanov, N. P. Johnson, A. V. Fokin, V. Y. Butko, H. M. Yates, M. E. Pemble, and C. M. S. Torres. "Enhancement of the Photonic Gap of Opal-based 3-D Gratings." Applied Physics Letters 70, 2091 (1997)

48. K. Yoshino, Y. Shimoda, Y. Kawagishi, K. Nakayama, and M. Ozaki. "Temperature Tuning of the Stop Band in Transmission Spectra of Liquid-crystal Infiltrated Synthetic Opal as Tunable Photonic Crystal." Applied Physics Letters. 75, 932 (1999)

PBG-Metallic Waveguides

49. W. Y. Leung, G. Tuttle, M. M. Sigalas, R. Biswas, K.-M. Ho, and C. M. Soukoulis. "Optimizing the Q Value in 3-D Metallic Photonic Band Gap Crystals." Journal of Applied Physics. 84, 4091 (1998)

50. C. Jin, B. Cheng, B. Man, D. Zhang, S. Ban, B. Sun, L. Li, X. Zhang, and Z. Zhang. "Two-dimensional Metallodielectric Photonic Crystal with a Large Band Gap." Applied Physics Letters. 75, 1201 (1999)

PBG-Magnetic Particle Introduction

51. M. Golosovsky, Y. Saado, and D. Davidov. "Self-Assembly of Floating Magnetic Particles into Ordered Structures: A Promising Route for the Fabrication of Tunable Photonic Band Gap Materials." Applied Physics Letters. 75, 4168 (1999)

PBG-Colloidal Systems

52. I. Inanc Tarhan and George H. Watson. "Photonic Band Structure of FCC Colloidal Crystals.” Physical Review Letters. 76, 315 (1996)

53. J. Wijnhoven and W. L. Vos. "Preparation of Photonic Crystals Made of Air Spheres in Titania." Science. 281, 802 (1998) 
54. G. Subramania, K. Constant, R. Biswas, M. M. Sigalas, and K.-M. Ho. “Optical Photonic Crystals Fabricated from Colloidal Systems." Applied Physics Letters. 74, 3933 (1999)

55. J. Ballato and E. Gulliver. "Photonic Band Engineering Through Tailored Microstructural Order.” Applied Physics Letters. 75, 1497 (1999)

56. P. V. Braun, R. W. Zehner, C. A. White, M. K. Weldon, C. Kloc, S. S. Patel, and P. Wiltzius. "Formation and Optical Spectroscopy of High Dielectric Contrast 3D Photonic Crystals." Submitted to Nature (1999)

57. G. Subramanian, V. N. Manoharan, J. D. Thorne, and D. J. Pine. “Ordered Macroporous Materials by Colloidal Assembly: A Possible Route to Photonic Band Gap Materials.” Advanced Materials. 11, 1261 (1999)

58. H. B. Sun, J. Song, Y. Xu, S. Matsuo, H. Misawa, G. Du, and S. Liu. "Growth and Property Characterizations of Photonic Crystal Structures Consisting of Colloidal Microparticles." Journal of the Optical Society of America B. 17, 476 (2000)

59. Younan Xia, Byron Gates, and Sang Hyun Park. "Fabrication of Three-Dimensional Photonic Crystals for Use in the Spectral Region from Ultraviolet to Near-Infrared." Journal of Lightwave Technology. 17, 1956 (1999)

60. Yu. A. Vlasov, M. Deutsch, and D. J. Norris. "Single-Domain Spectroscopy of SelfAssembled Photonic Crystals." Applied Physics Letters. 76, 1627 (2000)

Applications-Optical Waveguides

61. R. D. Meade, A. Devenyi, J. D. Joannopoulos, O. L. Alerhand, D. A. Smith, and K. Kash. "Novel Applications of Photonic Band Gap Materials: Low-Loss Bends and High Q Cavities." Journal of Applied Physics. 75, 4753 (1994)

62. P. Villeneuve and J. D. Joannopoulos. "Tricks of the Light." New Scientist. Aug. 26 page 26. (1995)

63. T. A. Birks, J. C. Knight, and P. St. J. Russell. "Endlessly Single-Mode Photonic Crystal Fiber." Optics Letters. 22, 961 (1997)

64. J. C. Knight, T. A. Birks, P. St. J. Russell, and J. P. de Sandro. "Properties of Photonic Crystal Fiber and the Effective Index Model." Journal of the Optical Society of America A. 15, 748 (1998)

65. S.-Y. Lin, E. Chow, V. Hietala, P. R. Villeneuve, and J. D. Joannopoulos.

"Experimental Demonstration of Guiding and Bending of Electromagnetic Waves in a Photonic Crystal.” Science. 282, 274 (1998) 
66. J. C. Knight, J. Broeng, T. A. Birks, and P. St. J. Russell. "Photonic Band Gap Guidance in Optical Fibers." Science. 282, 1476 (1998)

67. S. G. Johnson, C. Manolatou, S. Fan, P. R. Villeneuve, J. D. Joannopoulos, and H. A. Haus. "Elimination of Cross Talk in Waveguide Intersections." Optics Letters. 23, 1855 (1998)

68. R. F. Cregan, B. J. Mangan, J. C. Knight, T. A. Birks, P. St. J. Russell, P. J. Roberts, and D. C. Allan. "Single-Mode Photonic Band Gap Guidance of Light in Air." Science. 285, 1537 (1999)

69. J. Pendry. "Playing Tricks with Light." Science. 285, 1687 (1999)

70. C. Jin, B. Cheng, B. Man, Z. Li, D. Zhang, S. Ban, and B. Sun. "Band Gap and Wave Guiding Effect in a Quasiperiodic Photonic Crystal." Applied Physics Letters. 75, 1848 (1999)

71. D. Normile. "Cages for Light Go From Concept to Reality." Science. 286, 1500 (1999)

72. A. Chutinan, and S. Noda. "Highly Confined Waveguides and Waveguide Bends in 3D Photonic Crystal." Applied Physics Letters. 75, 3739 (1999)

73. Susumu Noda, Katsuhiro Tomoda, Noritsugu Yamamoto, and Alongkarn Chutinan. "Full Three-Dimensional Photonic Band gap Crystals at Near-Infrared Wavelengths." Science. 289, 604 (2000)

74. Masatoshi Tokushima, Hideo Kosaka, Akihisa Tomita, and Hirohito Yamada. "Lightwave Propagation Through a 120 Degree Sharply Bent Single-Line-Defect Photonic Crystal Waveguiede." Applied Physics Letters. 76, 952 (2000)

75. S. W. Leonard, H. M. van Driel, A. Birner, U. Gosele, and P. R. Villeneuve. "SingleMode Transmission in Two-Dimensional Macroporous Silicoan Photonic Crystal Waveguides." Optics Letters. 25, 1550 (2000)

Applications-Lasers/Diodes

76. F. De Martini, M. Marrocco, P. Mataloni, D. Murra, and R. Loudon. "Spontaneous and Stimulated Emission in the Thresholdless Microlaser." Journal of the Optical Society of America B. 10, 360 (1993)

77. D. Y. Chu, and S.-T. Ho. "Spontaneous Emission from Excitons in Cylindrical Dielectric Waveguides and the Spontaneous-Emission Factor of Microcavity Ring Lasers." Journal of the Optical Society of America B. 10,381 (1993) 
78. T. Erdogan, K. G. Sullivan, and D. G. Hall. "Enhancement and Inhibition of Radiation in Cylindrically Symmetric, Periodic Structures." Journal of the Optical Society of America B. 10, 391 (1993)

79. D. L. Bullock, C.-C. Shih, and R. S. Margulies. "Photonic Band Structure Investigation of 2-D Bragg Reflector Mirrors for Semiconductor Laser Mode Control." Journal of the Optical Society of America B. 10, 399 (1993)

80. M. Scalora, J. P. Dowling, C. M. Bowden, and M. J. Bloemer. "The Photonic Band Edge Optical Diode." Journal of Applied Physics. 76, 2023 (1994)

81. Y. A. Vlasov, K. Luterova, I. Pelant, B. Honerlage, and V. N. Astratov. "Enhancement of Optical Gain of Semiconductors Embedded in 3-D Photonic Crystals." Applied Physics Letters. 71, 1616 (1997)

82. P. Rigby and T. F. Krauss. "The Vs and Qs of Optical Microcavities." Nature. 390, 125 (1997)

83. M. Meier, A. Mekis, A. Dodabalapur, A. Timko, R. E. Slusher, J. D. Joannopoulos, and O. Nalamasu. "Laser Action from 2-D Distributed Feedback in Photonic Crystals." Applied Physics Letters. 74, 8 (1999)

84. R. K. Lee, O. J. Painter, B. D’Urso, A. Scherer, and A. Yariv. “ Measurement of Spontaneous Emission from a 2-D Photonic Band Gap Defined Microcavity at NearInfrared Wavelengths." Applied Physics Letters. 74, 1522 (1999)

85. O. Painter, R. K. Lee, A. Scherer, A. Yariv, J. D. O’Brien, P. D. Dapkus, and I. Kim. “2-D Photonic Band Gap Defect Mode Laser." Science. 284, 1819 (1999)

86. M. Imada, S. Noda, A. Chutinan, T. Tokuda, M. Murata, and G. Sasaki. “Coherent 2-D Lasing Action in Surface-Emitting Laser with Triangular-Lattice Photonic Crystal Structure." Applied Physics Letters. 75, 316 (1999)

87. M. Boroditsky, T. F. Krauss, R. Coccioli, R. Vrijen, R. Bhat, and E. Yablonovitch. "Light Extraction from Optically Pumped LED by Thin-Slab Photonic Crystals." Applied Physics Letters. 75, 1036 (1999)

88. O. J. A. Schueller, G. M. Whitesides, J. A. Rogers, M. Meier, and A. Dodabalapur. "Fabrication of Photonic Crystal Lasers by Nanomolding of Solgel Glasses." Applied Optics. 38, 5799 (1999)

89. M. Meier, A. Dodabalapur, J. A. Rogers, R. E. Slusher, A. Mekis, A. Timko, C. A. Murray, R. Ruel, and O. Nalamasu. "Emission Characteristics of 2-D Organic Photonic Crystal Lasers Fabricated by Replica Molding." Journal of Applied Optics. 86, 3502 (1999) 
90. K. M. Chen, A. W. Sparks, H.-C. Luan, D. R. Lim, K. Wada, and L. C. Kimerling. " $\mathrm{SiO}_{2} / \mathrm{TiO}_{2}$ Omnidirectional Reflector and Microcavity Resonator Via the Sol-Gel Method." Applied Physics Letters. 75, 3805 (1999)

91. F. De Martini and G. R. Jacobovitz. "Anomalous Spontaneous-Stimulated-Decay Phase Transition and Zero-Threshold Laser Action in a Microscopic Cavity." Physical Review Letters. 60, 1711 (1988)

92. J. P. Dowling, M. Scalora, M. J. Bloemer, and C. M. Bowden. "The Photonic Band Edge Laser: A New Approach to Gain Enhancement." Journal of Applied Physics. 75, $1896(1993)$

93. Peidong Yang, Gernot Wirnsberger, Howard C. Huang, Steven R. Cordero, Michail D. McGehee, Brian Scot, Tao Deng, George M. Whitesides, Bradley F. Chmelka, Steven K. Buratto, and Galen D. Stucky. "Mirrorless Lasing from Mesostructured Waveguides Patterned by Soft Lthography." Science. 287, 465 (2000)

Applications-Detectors

94. B. Temelkuran, E. Ozbay, J. P. Kavanaugh, G. Tuttle, and K.-M. Ho. " Resonant Cavity Enhanced Detectors Embedded in Photonic Crystals." Applied Physics Letters. 72, 2376 (1998)

95. J. Ballato, and A. James. "A Ceramic Photonic Crystal Temperature Sensor." Journal of the American Ceramic Society. 82, 2273 (1999)

96. J. Ballato. "Tailoring Visible Photonic Band gaps Through Microstructural Order and Coupled Material Effects in $\mathrm{SiO}_{2}$ Colloidal Crystals." Journal of the Optical Society of America. 17, 219 (2000)

97. A. Z. Genack and N. Garcia. "Electromagnetic Localization and Photonics." Journal of the Optical Society of America B. 10, 408 (1993)

Applications-Various

98. E. R. Brown, C. D. Parker, and E. Yablonovitch. "Radiation Properties of a Planar Antenna on a Photonic-Crystal Substrate." Journal of the Optical Society of America B. 10, 404 (1993)

99. V. Berger, O. Gauthier-Lafaye, and E. Costard. "Photonic Band Gaps and Holography.” Journal of Applied Physics. 82, 60 (1999)

100. S. Gupta, G. Tuttle, M. Sigalas, and K.-M. Ho. "Infrared Filters Using Metallic Photonic Band Gap Structures on Flexible Substrates." Applied Physics Letters. 71, 2412 (1997) 
101. D. F. Sievenpiper, C. F. Lam, and E. Yablonovitch. "2-D Photonic-Crystal VerticalCavity Array for Nonlinear Optical Image Processing." Applied Optics. 37, 2074 (1998)

102. F.-R. Yang, K.-P. Ma, Y. Qian, and T. Itoh. "A Uniplanar Compact Photonic-Band gap (UC-PBG) Structure and Its Applications for Microwave Circuits." IEEE Transactions on Microwave and Techniques. 47, 1509 (1999)

103. M. Thevenot, C. Cheype, A. Reineix, and B. Jecko. "Directive Photonic-Band gap Antennas." IEEE Transactions on Microwave and Techniques. 47, 2115 (1999)

104. M. M. Beaky, J. B. Burk, H. O. Everitt, M. A. Haider, and S. Venakides. "2-D Photonic Crystal Fabry-Perot Resonators with Lossy Dielectrics." IEEE Transactions on Microwave and Techniques. 47, 2085 (1999)

Laser Dyes - General

105. R. A. Keller. "Tunable Lasers for Chemists.” Chemtech. October, 627 (1973)

106. A. J. Finlayson, N. Peters, P. V. Kolinsky, and M. R. W. Venner. "Flashlamp Pumped Polymer Dye Laser Containing Rhodamine 6G." Applied Physics Letters. 72, 2153 (1998)

107. W. J. Wadsworth, S. M. Giffin, L. T. McKinnie, J. C. Sharpe, A. D. Woolhouse, T. G. Haskell, and G. J. Smith. "Thermal and Optical Properties of Polymer Hosts for Solid-state Dye Lasers." Applied Optics. 38, 2504 (1999)

Laser Dyes - Scattering Particles

108. N. M. Lawandy, R. M. Balachandran, A. S. L. Gomes, and E. Sauvaln. "Laser Action in Strongly Scattering Media." Nature. 368, 437 (1994)

109. W. Zhang, N. Cue, and K. M. Yoo. "Emission Linewidth of Laser Action in Random Gain Media.” Optics Letters. 20, 961 (1995)

110. M. A. Noginov, H. J. Caulfield, N. E. Noginova, and P. Venkateswarlu. "Line Narrowing in the Dye Solution with Scattering Centers." Optics Communications. 118, $430(1995)$

111. R. M. Balachandran, D. P. Pacheco, and N. M. Lawandy. "Laser Action in Polymeric Gain Media Containing Scattering Particles.” Applied Optics. 35, 640 (1996)

112. R. M. Balachandran, D. P. Pacheco, and N. M. Lawandy. "Photonic Textile Fibers." Applied Optics. 35, 1991 (1996) 
113. R. M. Balachandran, N. M. Lawandy, and J. A. Moon. "Theory of Laser Action in Scattering Gain Media.” Optics Letters. 22, 319 (1997)

114. G. Beckering, S. J. Zilker, and D. Haarer. "Spectral Measurements of the Emission from Highly Scattering Gain Media.” Optics Letters. 22, 1427 (1997)

115. S. Tanosaki, H. Taniguchi, K. Tsujita, B. Devaraj, and H. Inaba. "Highly Scattering Intralipid-10\% Assisted Lasing from Microdroplets with Acridine Orange Dye." Applied Optics. 37, 2379 (1998)

116. H. Z. Wang, F. L. Zhao, Y. J. He, X. G. Zheng, X. G. Huang, and M. M. Wu. "Lowthreshold Lasing of a Rhodamine Dye Solution Embedded with Nanoparticle Fractal Aggregates." Optics Letters. 23, 777 (1998)

117. S. V. Frolov, Z. V. Vardeny, K. Yoshino, A. Zakhidov, and R. H. Baughman. "Stimulated Emission in High-gain Organic Media." Physical Review B. 59, R5284 (1999)

118. G. Van Soest, M. Tomita, and A. Lagendijk. "Amplifying Volume in Scattering Media." Optics Letters. 24, 306 (1999)

119. H. Cao, Y. G. Zhao, S. T. Ho, E. W. Seelig, Q. H. Wang, and R. P. H. Chang. "Random Laser Action in Semiconductor Powder." Physical Review Letters. 82, 2278 (1999)

Laser Dyes - Opal infiltration

120. H. Yanagi, T. Hishiki, T. Tobitani, A. Otomo, and S. Mashiko. "Thin Film Lasing from a Dye-doped Silica/Titania Composite." Chemical Physics Letters. 332, 2925 (1998)

121. K. Yoshino, S. B. Lee, S. Tatsuhara, Y. Kawagishi, M. Ozaki, and A. A. Zakhidov. Observation of Inhibited Spontaneous Emission and Stimulated Emission of Rhodamine 6G in Polymer Replica of Synthetic Opal.” Applied Physics Letters. 73, 3506 (1998)

122. S. V. Frolov, Z. V. Vardeny, A. A. Zakhidov, R. H. Baughman. "Laser-like Emission in Opal Photonic Crystals." Optics Communications. 162, 241 (1999)

123. S. G. Romanov, T. Maka, C. M. S. Torres, M. Muller, and R. Zentel. "Photonic Bandgap Effects Upon the Light Emission from a Dye-Polymer-Opal Composite." Applied Physics Letters. 75, 1057 (1999) 
124. E. P. Petrov, V. N. Bogomolov, I. I. Kalosha, and S. V. Gaponenko. "Spontaneous Emission of Organic Molecules Embedded in a Photonic Crystal." Physical Review Letters. 81, 77 (1998)

PPV Laser Dye

125. C. Zhang, D. Braun, and A. J. Heeger. "Light-emitting Diodes from Partially Conjugated Poly(p-phenylene vinylene)." Journal of Applied Physics. 73, 5177 (1993)

126. M. Strukelj, T. M. Miller, F. Papadimitrakopoulos, and S. Son. "Effects of Polymeric Electron Transporters and the Structure of Poly(p-phenylene vinylene) on the Performance of Light-Emitting Diodes." Journal of the American Chemical Society. 117, $11976(1995)$

127. S. Son, A. Dodabalapur, A. J. Lovinger, and M. E. Galvin. "Luminescence Enhancement by the Introduction of Disorder into Poly(p-phenylene vinylene)." Science. 269, 376 (1995)

128. V. Cimrova and D. Neher. "Microcavity Effects in Single-layer Light-emitting Devices Based on Poly(p-phenylene vinylene)." Journal of Applied Physics. 79, 3299 (1996)

129. M. Esteghamatian, Z. D. Popovic, and G. Xu. "Carrier Generation Process in Poly(pphenylene vinylene) by Fluorescent Quenching and Delayed-Collection-Field Techniques." Journal of Physics Chemistry. 100, 13716 (1996)

130. A. C. Fou, O. Onitsuka, M. Ferreira, M. F. Rubner, and B. R. Hsieh. Fabrication and Properties of Light-emitting Diodes Based on Self-assembled Multilayers of Poly(pphenylene vinylene)." Journal of Applied Physics. 79, 7501 (1996)

131. O. Onitsuka, A. C. Fou, M. Ferreira, B. R. Hsieh, and M. F. Rubner. "Enhancement of Light Emitting Diodes Based on Self-assembled Heterostructures of Poly(pphenylene vinylene)." Journal of Applied Physics. 80, 4067 (1996)

132. M. Herold, J. Gmeiner, C. Drummer, M. Schwoerer. "Poly(p-phenylene vinylene) Light-Emitting Devices Prepared Via the Precursor Route onto Indium Tin Oxide and Fluorine-Doped Tin Dioxide Substrates.” Journal of Materials Science. 32, 5709 (1997)

133. J. C. Carter, I. Grizzi, S. K. Heeks, D. J. Lacey, S. G. Latham, P. G. May, O. Ruiz de los Panos, K. Pichler, C. R. Towns, and H. F. Wittmann. "Operating Stability of Light-Emitting Polymer Diodes Based on Poly(p-phenylene vinylene)." Applied Physics Letters. 71, 34 (1997) 
134. N. D. Kumar, M. P. Joshi, C. S. Friend, P. N. Prasad, and R. Burzynski. "OrganicInorganic Heterojunction Light Emitting Diodes Based on Poly(p-phenylene vinylene)/Cadmium Sulfide Thin Films.” Applied Physics Letters. 71, 1388 (1997)

135. E. Lebedev, Th. Dittrich, V. Petrova-Koch, S. Karg, and W. Brutting. "Charge Carrier Mobility in Poly(p-phenylene vinylene) Studied by the Time-of-Flight Technique." Applied Physics Letters. 71, 2686 (1997)

136. H. G. Lee, S. Kim, C. Hwang, V. Choong, Y. Park, Y. Gao, and B. R. Hsieh. "A Study of Poly(p-phenylene vinylene) and its Derivatives Using X-ray Photoelectron Spectroscopy." Journal of Applied Physics. 82, 4962 (1997)

137. M. Meier, S. Karg, K. Zuleeg, W. Brutting, and M. Schwoerer. "Determination of Trapping Parameters in Poly(p-phenylene vinylene) Light-Emitting Devices Using Thermally Stimulated Currents." Journal of Applied Physics. 84, 87 (1998)

138. J. A. Rogers, Z. Bao, and L. Dhar. "Fabrication of Patterned Electroluminescent Polymers that Emit in Geometries with Feature Sizes into the Submicron Range." Applied Physics Letters. 73, 294 (1998)

139. M. N. Shkunov, W. Gellermann, and Z. V. Vardeny. "Amplified Resonant Raman Scattering in Conducting Polymer Thin Films." Applied Physics Letters. 73, 2878 (1998)

140. G. Gigli, R. Rinaldi, C. Turco, P. Visconti, R. Cingolani, and F. Cacialli. "Holographic Nanopatterning of the Organic Semiconductor Poly(p-phenylene vinylene)." Applied Physics Letters. 73, 3926 (1998)

141. J.-H. Hsu, W Fann, P.-H. Tsao, K.-R. Chuang, and S.-A. Chen. "Fluorescence from Conjugated Polymer Aggregates in Dilute Poor Solution." Journal of Physical Chemistry A. 103, 2375 (1999)

142. J. Morgado, F. Cacialli, J. Gruner, N. C. Greenham, and R. H. Friend. "Luminescence Properties of Poly(p-phenylene vinylene): Role of the Conversion Temperature on the Photoluminescence and Electroluminescence efficiencies." Journal of Applied Physics. 85, 1784 (1999)

Mold Production Method

143. A. Kumar and G. M. Whitesides. "Features of Gold Having Micrometer to Centimeter Dimensions can be Formed Through a Combination of Stamping with an Elastomeric Stamp and an Alkanethiol "Ink" Followed by Chemical Etching." Applied Physics Letters. 63, 2002 (1993) 
144. A. Kumar, H. A. Biebuyck, and G. M. Whitesides. "Patterning Self-Assembled Monolayers: Applications in Materials Science.” Langmuir. 10, 1498 (1994)

145. X.-M. Zhoa, Y. Xia, and G. M. Whitesides. "Fabrication of 3-D Micro-Structures: Microtransfer Molding." Communications. June $3^{\text {rd }}$ (1996)

146. K. P. Constant, and J. Huang. "Using Microtransfer Molding to Produce Photonic Band Gap Materials.” Carver Trust Grand Report. (2000)

General References

147. Kittel, Charles. Introduction to Solid State Physics $7^{\text {th }}$ Edition. John Wiley \& Sons, New York; 1996.

148. Joannopoulos, John, Meade, Robert, and Winn, Joshua. Photonic Crystals: Molding the Flow of Light. Princeton University Press; 1995. 


\section{ACKNOWLEDGEMENTS}

I would like to thank my graduate committee: Dr. Kai-Ming Ho, Dr. David Cann, and Dr. Kristen Constant. I would like to especially thank Kristen Constant for helping me so much through my six years of college at Iowa State University.

I would also like to thank the remainder of our research group: Chan-Hwan Kim, Henry Kang, Wai Leung, Rana Biswas,

Special thanks to Henry Kang, Chan-Hwan Kim, and Wai Leung, with whom I worked closely towards our goals.

This work was performed at Ames Laboratory under Contract No. W-7405-Eng-82 with the U.S. Department of Energy. The United States government has assigned the DOE Report number IS-T 1981 to this thesis. 